\title{
Análise Bayesiana de Modelos para Dados Binários Correlacionados
}

\author{
Vanderly Janeiro
}

Orientador: Prof. Dr. Jorge Alberto Achcar

Dissertação apresentada ao Instituto de Ciências Matemáticas e de Computação - ICMC-USP, para o exame de qualificação, como parte dos requisitos para obtenção do título de Mestre em Ciências - Área: Ciências de Computação e Matemática Computacional. 


\section{Agradecimentos}

Sobretudo agradeço a Deus por me conceder a vida e estar aqui realizando mais este sonho.

Ao Prof. Dr. Jorge Alberto Achcar, por me confiar este trabalho, e pela orientação no decorrer de seu andamento, e especialmente por ter compartilhado comigo a sua grande experiência.

Aos professores que me auxiliaram e incentivaram para que eu pudesse chegar a mais esta etapa da minha vida.

Aos professores desta instituição: Profa. Dra. Maria Creusa B. Salles, Prof. Dr. Josemar Rodrigues, Prof. Dr. Marinho Gomes de Andrade Filho pela dedicação e empenho ao ensino.

Ao Prof. Dr. José Galvão Leite e ao Prof. Dr. Marinho pelas sugestões dadas em meu exame de qualificação, que vieram a contribuir muito com esta dissertação e com meu crescimento.

À todos os meus amigos que direta ou indiretamente contribuíram para que este trabalho fosse realizado, e em especial a Lucimary Afonso dos Santos que nos meus momentos de desespero manteve-se sempre do meu lado.

Com muito amor agradeço aos meus pais e meus irmãos por acreditarem em mim e sempre estarem ao meu lado.

Aos funcionários do ICMC pela dedicação às pessoas deste departamento.

E finalmente, à CAPES pelo apoio financeiro. 


\begin{abstract}
In this dissertation, we develop a Bayesian analysis of regression models for correlated binary data in the presence of covariates, including the case with replicates. We consider probit and logistic regression models for correlated binary data assuming random effects with a mixture of normal distributions, since this model have great flexibility to the fitted for correlated binary data. We also present some considerations for the case with replicates. We assume informative prior distributions for the parameters of the model and we use Gibbs sampling and Metropolis-Hastings algorithms to get Monte Carlo estimates for the posterior quantities of interest. We also present some considerations for the selection of models using discrepancy measures between the fitted model and the data (Pearson residuals) and using the predictive densities (Bayes factor) estimated by MCMC (Markov Chain Monte Carlo). We present a numerical example to illustrate the proposed methodology.
\end{abstract}




\section{Resumo}

Nesta dissertação, desenvolvemos uma análise Bayesiana de modelos de regressão para dados binários correlacionados com covariáveis, podendo ocorrer réplicas. Assumimos os modelos de regressão logístico e probito para dados binários correlacionados considerando efeitos aleatórios com uma mistura de distribuições normais, pois este modelo tem uma grande flexibilidade para ser ajustado aos dados binários correlacionados em muitas aplicações. Também fazemos algumas considerações aos casos onde podem ocorrer repetições das observações ou réplicas. Assumimos distribuições a priori informativas para os parâmetros do modelo e consideramos os algoritmos Gibbs sampling e MetropolisHastings, para obter as estimativas de Monte Carlo para as quantidades a posteriori de interesse. Apresentamos também algumas considerações na seleção de modelos utilizando uma medida da discrepância entre o modelo ajustado e os dados (resíduo de Pearson) e utilizando as densidades preditivas (fator de Bayes) estimadas por MCMC (Monte Carlo em Cadeias de Markov). Apresentamos um exemplo númerico para ilustrar os métodos propostos. 


\section{Sumário}

1 INTRODUÇĀO 1

1.1 Motivações, Modelos e Objetivos .................. 3

1.1.1 Alguns Modelos Considerados na Literatura para Análise de Dados Binários Correlacionados . . . . . . . . . . . . 5

1.2 Organização da Dissertação . . . . . . . . . . . . . . . . . 9

2 ANÁLISE DO MODELO DE PRENTICE 11

2.1 Função de Verossimilhança . . . . . . . . . . . . . . . . . 12

2.2 Análise Clássica do Modelo . . . . . . . . . . . . . . . 13

2.3 Análise Bayesiana do Modelo . . . . . . . . . . . . . 16

2.4 Análise Bayesiana Hierárquica Assumindo Efeitos Aleatórios Normais . . . 19

2.5 Modelo de Efeitos Aleatórios com uma Mistura de Distribuiçōes Normais . 21

3 ANÁLISE DO MODELO PROBITO

3.1 Análise Clássica do Modelo . . . . . . . . . . . . . . . . 28

3.2 Análise Bayesiana do Modelo . . . . . . . . . . . . . . 30

3.3 Análise Bayesiana Assumindo uma Distribuição Normal para os efeitos

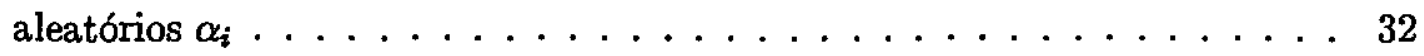

3.4 Análise Bayesiana Assumindo uma Mistura de Normais para $\alpha_{i} \ldots \ldots$. . . 34

4 DADOS BINÁRIOS CORRELACIONADOS COM RÉPLICAS 39

4.1 Estimadores de Máxima Verossimilhança. . . . . . . . . . . . . . . 40

4.2 Análise Bayesiana Assumindo $\alpha_{i}=\alpha \ldots \ldots \ldots \ldots$. . . . . . . 41

$4.3 \quad$ Análise Bayesiana Assumindo $\alpha_{i} \sim N\left(0, \sigma_{\alpha}^{2}\right) \ldots \ldots \ldots \ldots$

4.4 Análise Bayesiana Assumindo uma Mistura de Normais para $\alpha_{i}$. . . . . . 44 
$5 \quad$ SELEÇÃO DE MODELOS $\quad 47$

5.1 Resíduos de Pearson . . . . . . . . . . . . . . . . 47

5.2 Um Procedimento Bayesiano para Verificação da adequação de Modelos Fator de Bayes . . . . . . . . . . . . . . . 48

5.2.1 Aproximação de Monte Carlo - (MC) . . . . . . . . . . . . . . 49

5.2.2 Aproximações de Monte Carlo em Cadeias de Markov - (MCMC) 49

5.2 .3 A Escolha do Modelo .................. 51

6 APLICAÇÕES NUMÉRICAS

6.1 Ilustração Numérica para o Capítulo $2 \ldots \ldots \ldots$

6.1.1 EMV para os Parâmetros do Modelo com Ligação Logística . . . . 55

6.1 .2 Aplicação do Modelo $1 \ldots \ldots$. . . . . . . . . . . . . 56

6.1.3 Aplicação do Modelo 2 . . . . . . . . . . . . . . . . . 61

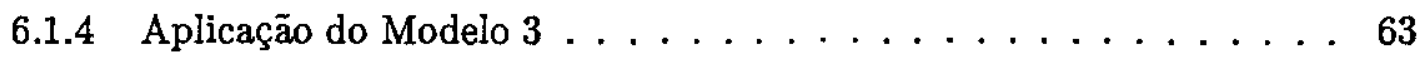

6.1.5 Comparação dos Modelos 1, 2 e $3 \ldots \ldots 6$

6.2 Ilustração Numérica para o Capítulo $3 \ldots \ldots$. . . . . . . . . . . 69

6.2.1 EMV para os Parâmetros do Modelo com Ligação Probito . . . . . 70

6.2 .2 Aplicação do Modelo $4 \ldots$. . . . . . . . . . . . . . . 70

6.2 .3 Aplicação do Modelo $5 \ldots \ldots . \ldots . \ldots 73$

6.2 .4 Aplicação do Modelo $6 \ldots \ldots \ldots$. . . . . . . . . . 76

6.2 .5 Comparação dos Modelos 4, 5 e $6 \ldots \ldots$. . . . . . . . 79

6.3 Ilustração Numérica para o Capítulo $4 \ldots \ldots$. . . . . . . . . . 81

6.3.1 EMV para os Parâmetros do Modelo com Ligação Logística Considerando Réplicas . . . . . . . . . . . . . . . . . . . 84

6.3.2 Aplicação do Modelo 7 . . . . . . . . . . . . . . . . 84

6.3 .3 Aplicação do Modelo $8 \ldots \ldots$. . . . . . . . . . 86

6.3 .4 Aplicação do Modelo $9 \ldots \ldots$. . . . . . . . . . . 88

6.3.5 Comparação dos Modelos 7,8 e $9 \ldots \ldots$. . . . . . . . 90

6.3.6 EMV para os Parâmetros do Modelo com Ligação Probito Considerando Réplicas . . . . . . . . . . . . . . . . . . 92

6.3 .7 Aplicação do Modelo 10 . . . . . . . . . . . . . . . . . 92

6.3 .8 Aplicação do Modelo $11 \ldots \ldots$. . . . . . . . . . . . . 94 
6.3 .9 Aplicação do Modelo $12 \ldots \ldots$. . . . . . . . . . . . . 97

6.3.10 Comparação dos Modelos 10, 11 e 12 . . . . . . . . . . . . . . 99

7 CONCLUSŌES E CONSIDERAÇŌES FUTURAS 101

Apêndices

102

A ALGORITMO GIBBS SAMPLING

103

B ALGORITMO DE METROPOLIS-HASTINGS

105

C O CRITÉRIO DE CONVERGÊNCIA DE GELMAN E RUBIN (1992)107

D PROGRAMA COMPUTACIONAL PARA O MODELO 3

109

Referências Bibliográficas

116 


\section{Capítulo 1}

\section{INTRODUÇÃO}

Supor que associado a cada indivíduo ou unidade experimental temos uma observação onde a resposta assume um, de dois possíveis valores (sucesso ou fracasso). Isto pode ocorrer em muitas áreas de interesse. Por exemplo:

- um componente eletrônico é defeituoso, ou não é defeituoso;

- um experimento agronômico tem por objetivo verificar se uma determinada semente germina ou não em diferentes condições experimentais (temperatura, umidade, adubação, etc.) controladas durante o experimento;

- um animal em teste morre de uma dose específica de veneno, ou vive;

Nesses exemplos os resultados de interesse nas unidades observadas assumem apenas dois valores possíveis.

Representamos a $i$-ésima observação ou resposta, por uma variável aleatória $Y_{i}$, que sem perda de generalidade pode ser codificada pelos valores 0 ou 1 . Frequentemente tem sido conveniente definir $Y_{i}=1$ como sucesso e $Y_{i}=0$ como fracasso (distribuição de Bernoulli com probabilidade de sucesso $\theta_{i}$ ); trata-se portanto de uma variável dicotômica, (binária), tendo probabilidades:

$$
\begin{aligned}
& \operatorname{Pr}\left[Y_{i}=1\right]=\theta_{i} \\
& \operatorname{Pr}\left[Y_{i}=0\right]=1-\theta_{i},
\end{aligned}
$$

com média e variância dadas por,

$$
\begin{aligned}
\mathbb{E}\left(Y_{i}\right) & =\theta_{i} \\
\operatorname{Var}\left(Y_{i}\right) & =\theta_{i}\left(1-\theta_{i}\right) .
\end{aligned}
$$


Em estudos como estes, estamos interessados em verificar o comportamento da probabilidade de sucesso em função de um vetor de variáveis ou covariáveis explicativas $\left\{X_{1}, X_{2}, \ldots, X_{k}\right\}$. Num planejamento de experimentos, este vetor de cováriáveis incluem usualmente várias variáveis indicadoras associadas com blocos e fatores de tratamento, junto com informações quantitativas sobre vários aspectos do material experimental. Uma análise estatística em geral investiga a relação entre a probabilidade $\theta=\theta(\underset{\sim}{X})$ e as variáveis explicativas $\underset{\sim}{X}=\left(X_{1}, X_{2}, \ldots, X_{k}\right)$.

Para investigar a relação entre a probabilidade de resposta $\theta$ e o vetor de covariadas $\underset{\sim}{X}$, é conveniente construir um modelo capaz de discriminar o efeito de $\theta$ pela transformação em $\left(X_{1}, X_{2}, \ldots, X_{k}\right)$. Na prática, este modelo usualmente incorpora suposiçōes tais como correlação zero ou independência, falta de interação e linearidade.

Modelos lineares fazem um importante papel tanto em trabalhos teóricos quanto aplicados. Supor inicialmente que a dependência de $\theta$ sobre $\left(X_{1}, X_{2}, \ldots, X_{k}\right)$ ocorre através da combinação linear

$$
\eta=\alpha+\sum_{j=1}^{k} X_{j} \beta_{j}
$$

de coeficientes desconhecidos $\beta=\left(\beta_{1}, \ldots, \beta_{k}\right)$. De (1.1), temos $-\infty<\eta<\infty$. Assim, expressar $\theta$ como combinação linear (1.1) seria inconsistente com as leis de probabilidade, pois $\theta$ estaria fora do intervalo [0;1]. Uma forma alternativa para evitar este problema é o uso de uma transformação $h(\theta)$ que mapeie o intervalo unitário sobre a reta real $(-\infty, \infty)$. Duas funçōes de ligação são comumente usadas na prática:

1) Logito ou função logística

$$
h_{1}(\theta)=\log \left(\frac{\theta}{1-\theta}\right)=\alpha+\sum_{j=1}^{k} X_{j} \beta_{j}
$$

2) Probito ou função Normal inversa

$$
h_{2}(\theta)=\Phi^{-1}(\theta)=\alpha+\sum_{j=1}^{k} X_{j} \beta_{j}
$$

onde

$$
\Phi(t)=\frac{1}{\sqrt{2 \pi}} \int_{-\infty}^{t} \exp \left\{-\frac{u^{2}}{2}\right\} d u
$$


De (1.2) temos,

$$
\begin{aligned}
\frac{\theta}{1-\theta} & =\exp \left(\alpha+\sum_{j=1}^{k} X_{j} \beta_{j}\right) \\
\theta & =\frac{\exp \left(\alpha+\sum_{j=1}^{k} X_{j} \beta_{j}\right)}{1+\exp \left(\alpha+\sum_{j=1}^{k} X_{j} \beta_{j}\right)} .
\end{aligned}
$$

Em (1.3) temos que:

$$
\theta=\Phi\left(\alpha+\sum_{j=1}^{k} X_{j} \beta_{j}\right)
$$

De forma geral, supor um vetor de variáveis explicativas $\underset{\sim}{X}$ e uma variável latente $W$ com função de distribuição acumulada $F(w ; x)$.

Consideremos que $Y=1$ se e somente se $W>0$, isto é,

$$
\theta=\operatorname{Pr}[Y=1 ; x]=1-F(0 ; x)
$$

Se considerarmos agora que a distribuição de $W$ é a função logística, temos:

$$
F(0 ; x)=\left(1+\exp \left(\alpha+\sum_{j=1}^{k} X_{j} \beta_{j}\right)\right)^{-1}=\operatorname{Pr}[Y=0 ; x]
$$

Então, $\quad \theta=\operatorname{Pr}[Y=1 ; x]=1-F(0 ; x)=\frac{\exp \left(\alpha+\sum_{j=1}^{k} X_{j} \beta_{j}\right)}{1+\exp \left(\alpha+\sum_{j=1}^{k} X_{j} \beta_{j}\right)}$

Considerando $W$ com distribuição $W \sim N\left(\alpha+\sum_{j=1}^{k} X_{j} \beta_{j}, 1\right)$, temos,

$$
p=\Phi\left(\alpha+\sum_{j=1}^{k} X_{j} \beta_{j}\right)
$$

\subsection{Motivações, Modelos e Objetivos}

Frequentemente quando duas ou mais medidas são tomadas ao mesmo tempo para alguma unidade experimental, indivíduo, ou quando medidas repetidas são tomadas para mesma unidade experimental temos dados binários longitudinais. Normalmente assumimos que tais observações binárias para $n$ unidades experimentais são independentes. Considerando que $Y_{i}$ assume resposta 0 ou $1, Y_{i}$ tem distribuição de Bernoulli com probabilidade de sucesso $\theta_{i}$, então métodos de regressão padrões são inadequados por varias razōes: 
(i) as covariáveis podem ser fixas para todos os tempos ou variar nos tempos;

(ii) existe o fator tempo;

(iii) observações repetidas para a mesma unidade experimental tendem a serem correlacionadas.

O uso dos modelos anteriores da forma com que foram construídos pode não ser coerente, pois em muitas aplicações temos dados binários correlacionados, já que para um mesmo indivíduo são feitas mais de uma medida. Muitos modelos tem sido propostos para ajustar modelos de regressão com variáveis binárias correlacionadas (ver por exemplo, Haseman \& Kupper, 1979).

Alguns modelos podem ser generalizados para permitir regressão de variáveis que caracterize cada observação binária correlacionada ou bloco o que permite estudar a relação entre várias covariáveis e medidas de efeitos dicotômicos. Em geral obter estimadores de máxima verossimilhança é trabalhoso e requer resoluções de equações que não tem solução analítica. Procedimentos Bayesianos podem evitar a necessidade dessas soluções numéricas usando técnicas de Monte Carlo em Cadeias de Markov (MCMC) (ver por exemplo, Gelfand, A.E. \& Smith, A.F.M., 1990), em particular o algoritmo Gibbs Sampling o algoritmo Metropolis-Hastings. Além disso no método Bayesiano é possível incorporar ao modelo um conhecimento prévio a respeito do parâmetro de interesse para a análise, isso porque na inferência Bayesiana o parâmetro é considerado uma variável aleatória, com uma densidade de probabilidade chamada de distribuição a priori do parâmetro.

O algoritmo Gibbs Sampling é um método de amostragem, para se obter amostras de uma distribuição de interesse, que explora as distribuições condicionais completas através de um algoritmo iterativo que define uma cadeia de Markov (ver Apêndice A). O algoritmo Metropolis-Hastings é um usado quando algum componente do modelo não possui distribuições condicionais padronizadas (ver Apêndice B).

Prentice (1988) apresenta uma revisāo completa de várias estratégias de modelagem usando análise de regressão linear generalizada de dados binários correlacionados com associação de covariáveis a cada resposta binária. Seguindo Liang e Zeger (1986) e Zeger e Liang (1986), Prentice considera o uso de equação de estimação generalizada 
(GEE) aproximada para obter estimadores consistentes e assintoticamente normais para os coeficientes de regressão.

Dey e Chen (1996) apresentam uma análise Bayesiana dos modelos propostos por Prentice (1988) e também por Ochi e Prentice (1984).

Nesta dissertação, exploramos diferentes estratégias de modelagem para a análise de dados binários correlacionados sob o enfoque Bayesiano, considerando métodos de Monte Carlo em Cadeias de Markov (MCMC) para simular as distribuições a posteriori de interesse. Para discriminar possíveis modelos usados para dados binários, usamos os dois métodos a seguir:

- Resíduos de Pearson;

- Fator de Bayes.

O fator de Bayes será aproximado pelas amostras geradas pelo método MCMC (ver por exemplo, Kass, R.E. \& Raftery, A.E., 1995; Gelfand, et al., 1992; Dey, et al.,1995).

\subsubsection{Alguns Modelos Considerados na Literatura para Análise de Dados Binários Correlacionados}

Os modelos considerados para análise de dados binários correlacionados podem ser classificados em dois grupos. O primeiro grupo apresenta modelos logísticos binários generalizados para dados multivariados, considerando uma particular representação parametrizada para as correlaçōes que usam a noção de efeitos aleatórios na estrutura de regressão logística em termos de pairwise odds ratio; o segundo grupo de modelos é obtido introduzindo uma função de ligação que usa uma função de distribuição cumulativa inversa. Modelos do tipo Probito Multivariado (MVP) e $t$ de Ligação Multivariada (MVT) são modelos neste grupo. O modelo MVP foi introduzido por Ashford e Sowden (1970) e também considerado por Amemiya (1985). Recentemente Chib e Greenberg (1995) usaram o modelo MVP numa abordagern Bayesiana.

Inicialmente introduzimos algumas notações que serão usadas no decorrer deste trabalho. Suponha que observamos uma resposta binária $Y_{i j}(0$ ou 1$)$ na $i$-ésima observação e $j$-ésima variável e temos $x_{i j}=\left(x_{i j 1}, x_{i j 2}, \ldots, x_{i j p_{j}}\right)$ correspondendo a linha do vetor de regressão de dimensão $p_{j} \operatorname{com} i=1,2, \ldots, n$ e $j=1,2, \ldots, J$. (Note que 
$x_{i j 1}$ pode ser igual a 1 , o qual corresponde ao intercepto). Seja $Y_{i}=\left(Y_{i 1}, Y_{i 2}, \ldots, Y_{i J}\right)^{\prime}$ e assumimos que $Y_{i 1}, Y_{i 2}, \ldots, Y_{i J}$ são dependentes e $Y_{1}, Y_{2}, \ldots, Y_{n}$ são independentes. Sejam $y_{i}=\left(y_{i 1}, y_{i 2}, \ldots, y_{i J}\right)^{\prime}$ e $y=y_{1}, y_{2}, \ldots, y_{n}$ os dados observados. Também seja $\beta_{j}=\left(\beta_{1 j}, \beta_{2 j}, \ldots, \beta_{p_{j} j}\right)^{\prime}$ um vetor coluna de coeficientes de regressão com dimensão $p_{j}$ para $j=1,2, \ldots, J$, correspondente à covariável $x_{i j}$, e $\beta=\left(\beta_{1}^{\prime}, \beta_{2}^{\prime}, \ldots, \beta_{J}^{\prime}\right)^{\prime}$.

\section{A) Modelos do Primeiro Grupo}

\section{(i) Modelos de Mistura e Estratificado}

Com a forma de modelos de mistura e estratificação considerado em Prentice (1988), assumir o modelo de regressão logistico binário para $Y_{i j}$ dado $x_{i j}$, por,

$$
P\left(Y_{i j}=y_{i j} \mid \alpha_{i}, \beta_{j}, x_{i j}\right)=\frac{\exp \left\{\left(\alpha_{i}+x_{i j} \beta_{j}\right) y_{i j}\right\}}{1+\exp \left(\alpha_{i}+x_{i j} \beta_{j}\right)}
$$

onde $\alpha_{i}$ denota um efeito aleatório na $i$-ésima observação, o qual especifica a correlação entre $Y_{i 1}, Y_{i 2}, \ldots, Y_{i J}$. Assumir também,

$$
\alpha_{i} \stackrel{i i d}{\sim} N\left(0, \sigma_{\alpha}^{2}\right)
$$

O modelo acima também é conhecido na literatura como um modelo log normal.

\section{(ii) Modelos Condicionais}

Em algumas aplicações de dados binários correlacionados, há uma ordem natural dos indivíduos dentre os blocos e os coeficientes de regressão nos modelos que podem caracterizar dependências de interesse. Como segundo modelo considerar um modelo de regressão logística binária de efeitos aleatórios condicionais simétricos (ver por exemplo, Prentice 1988), o qual é definido por,

$$
\begin{array}{r}
P\left(Y_{i 1}=y_{i 1}, Y_{i 2}=y_{i 2}, \ldots, Y_{i J}=y_{i J} \mid \alpha_{i}, \beta, x_{i 1}, \ldots, x_{i J}\right) \\
=\frac{\exp \left\{\sum_{j=0}^{\dot{y}_{i}-1} \alpha_{i}(j)+\sum_{j=1}^{J} x_{i j} \beta_{j} y_{i j}\right\}}{\sum_{y_{i 1}^{*}=0}^{1} \sum_{y_{i 2}^{*}=0}^{1} \cdots \sum_{y_{i j}^{*}=0}^{1} \exp \left\{\sum_{j=0}^{\dot{y}_{j}^{*}-1} \alpha_{i}(j)+\sum_{j=1}^{J} x_{i j} \beta_{j} y_{i j}^{*}\right\}},
\end{array}
$$

onde $\dot{y}_{i}=\sum_{j=1}^{J} y_{i j}$ e $\dot{y}_{i}^{*}=\sum_{j=1}^{J} y_{i j}^{*}$. Em (1.6), $\alpha_{i}(j)$ é escolhido para ser um 
polinômio em $j$. Por exemplo, a escolha $\alpha_{i}(j)=\alpha_{i}$ leva ao modelo,

$$
\begin{aligned}
& P\left(Y_{i 1}=y_{i 1}, Y_{i 2}=y_{i 2}, \ldots, Y_{i J}=y_{i J} \mid \alpha_{i}, \beta, x_{i 1}, \ldots, x_{i J}\right) \\
= & \frac{\exp \left\{\alpha_{i} \dot{y}_{i}+\sum_{j=1}^{J} x_{i j} \beta_{j} y_{i j}\right\}}{\sum_{y_{i 1}^{*}=0}^{1} \sum_{y_{i 2}^{*}=0}^{1} \cdots \sum_{y_{i J}^{*}=0}^{1} \exp \left\{\alpha_{i} \dot{y}_{i}^{*}+\sum_{j=1}^{J} x_{i j} \beta_{j} y_{i j}^{*}\right\}}
\end{aligned}
$$

onde $\alpha_{i} \stackrel{i i d}{\sim} N\left(0, \sigma_{\alpha}^{2}\right)$

\section{B) Alguns Modelos do Segundo Grupo}

\section{(i) Modelo Probito Multivariado}

Prentice (1988) considera a distribuição conjunta para $Y_{i 1}, Y_{i 2}, \ldots, Y_{i J}$ com marginais idênticas, o qual é essencialmente uma versão de modelos binários correlacionados. Aqui, consideramos o modelo probito multivariado (MVP); (ver por exemplo, Chib \& Greenberg, 1995).

Introduzimos um vetor $J$-dimensional $w_{i}=\left(w_{i 1}, w_{i 2}, \ldots, w_{i J}\right)^{\prime}$ tal que

$$
Y_{i j}= \begin{cases}1 & \text { se } w_{i j}>0 \\ 0 & \text { se } w_{i j} \leq 0\end{cases}
$$

e assumimos que

$$
w_{i} \stackrel{i i d}{\sim} N\left(X_{i} \beta, \Sigma\right)
$$

onde

$$
X_{i}=\left(\begin{array}{cccc}
x_{i 1} & 0 & \cdots & 0 \\
0 & x_{i 2} & \cdots & 0 \\
\vdots & \vdots & \ddots & \vdots \\
0 & 0 & \cdots & x_{i J}
\end{array}\right) .
$$

Em (1.9), consideramos $\Sigma=\left(\rho_{i j *}\right)_{J \times J}$ uma matriz de correlação tal que $\rho_{i j}=1$ para assegurar a identificabilidade dos parâmetros (ver Chib \& Greenberg, 1995, para detalhes). Portanto, a distribuição de $w_{i}$ determina a distribuiçāo conjunta de $Y_{i}$ por (1.8) e a matriz de correlação $\Sigma$ captura as correlaçōes entre $Y_{i j}$ 's. Especificamente, temos, 


$$
\begin{gathered}
P\left(Y_{i 1}=y_{i 1}, Y_{i 2}=y_{i 2}, \ldots, Y_{i J}=y_{i J} \mid, \beta, \Sigma, X_{i}\right) \\
=\int_{A_{1}} \int_{A_{2}} \ldots \int_{A_{J}}\left(\frac{1}{\sqrt{2 \pi}}\right)^{J} \frac{1}{|\Sigma|^{\frac{1}{2}}} \exp \left\{-\frac{1}{2}\left(w_{i}-X_{i} \beta\right)^{\prime} \Sigma^{-1}\left(w_{i}-X_{i} \beta\right)\right\} d w_{i}
\end{gathered}
$$

onde $A_{j}$ é um intervalo tal que

$$
A_{j}=\left\{\begin{array}{cc}
(-\infty, 0] & \text { se } y_{i j}=0 \\
(0, \infty) & \text { se } y_{i j}=1
\end{array}\right.
$$

(ii)Modelos $t$ de Ligação Multivariado

Uma generalização dos modelos probito multivariados são modelos $t$ multivariado (MVT). Semelhante aos modelos MVP, introduzimos um vetor aleatório $J$ dimensional $w_{i}=\left(w_{i 1}, w_{i 2}, \ldots, w_{i J}\right)^{\prime}$ tal que

$$
Y_{i j}= \begin{cases}1 & \text { se } w_{i j}>0 \\ 0 & \text { se } w_{i j} \leq 0\end{cases}
$$

e assumimos que

$$
w_{\mathrm{i}}^{\mathrm{i} i d} \stackrel{i}{\sim} t_{v}\left(X_{\mathrm{i}} \beta, \Sigma\right)
$$

onde $t_{v}\left(X_{\mathbf{i}} \beta, \Sigma\right)$ tem uma função de densidade de probabilidade

$$
\pi\left(w_{i} \mid v, X_{i} \beta, \Sigma\right)=\frac{\Gamma\left(\frac{1}{2}(v+J)\right)}{(\pi v)^{\left(\frac{1}{2}\right)^{J}} \Gamma\left(\frac{1}{2} v\right)|\Sigma|^{\frac{1}{2}}}\left(1+v^{-1}\left(w_{i}-X_{i} \beta\right)^{\prime} \Sigma^{-1}\left(w_{i}-X_{i} \beta\right)\right)^{-\frac{1}{2}(v+J)}
$$

Note que um caso especial de (1.13) com $v=1$ é denominado distribuição de Cauchy multivariada e outro caso especial de (1.13) com $v \longrightarrow \infty$ é a distribuição normal multivariada (1.9). As distribuições conjuntas de $Y_{i}$ são dadas por

$$
P\left(Y_{i 1}=y_{i 1}, Y_{i 2}=y_{i 2}, \ldots, Y_{i J}=y_{i J} \mid, \beta, \Sigma, v, X_{i}\right)=\int_{A_{1}} \int_{A_{2}} \cdots \int_{A_{J}} \pi\left(w_{i} \mid v, X_{i} \beta, \Sigma\right) d w_{i}
$$

onde $\pi\left(w_{i} \mid v, X_{i} \beta, \Sigma\right)$ e $A_{j}$ são dados em (1.13) e (1.11) respectivamente. Para simplificação podemos considerar $v$ fixado, o que facilita a análise Bayesiana do modelo. 


\subsection{Organização da Dissertação}

Esta dissertação encontra-se dividida em 6 capítulos. Neste capítulo introdutório apresentamos alguns modelos para dados binários sem correlaçāo, as motivaçōes e os obje- . tivos do trabalho, e ainda alguns modelos para dados binários correlacionados encontrados na literatura.

No capítulo 2, apresentamos uma análise do modelo (1.4) proposto por Prentice (1988), que é um modelo logístico generalizado. Para este modelo consideramos inicialmente efeitos fixos, ou seja, sem assumir correlação entre as observações, e apresentamos uma análise clássica e uma análise Bayesiana do modelo. A seguir, tratamos o modelo considerando efeitos aleatórios (1.5) onde $\alpha_{i}, i=1, \ldots, n$ captura a correlação entre as respostas, sob uma análise Bayesiana hierárquica, e ainda considerando uma mistura de distribuições normais.

Como uma alternativa ao modelo logístico (1.4) apresentamos no capítulo 3 , o modelo probito generalizado, e sobre este modelo fizemos as mesmas considerações feitas ao modelo logístico no capítulo 2, para desenvolver uma análise Bayesiana usando métodos MCMC.

Para análise de dados onde temos réplicas de unidades experimentais ou frequências nas observações, consideramos no capítulo 4 o modelo logístico e o modelo probito incorporando as réplicas das unidades experimentais, e desenvolvemos uma análise Bayesiana para este caso.

Com a finalidade de verificar qual ou quais modelos são mais adequados para fazer inferências sobre dados binários correlacionados, apresentamos no Capítulo 5 alguns procedimentos para discriminação dos modelos propostos.

Num capítulo 6, introduzimos exemplos de aplicação para ilustrar a metodologia proposta nesta dissertação. 
10 


\section{Capítulo 2}

\section{ANÁLISE DO MODELO DE PRENTICE}

Neste capítulo inicialmente introduzimos uma análise estatística do modelo logístico de Prentice (1988), sob o ponto de vista clássico, usando estimadores de máxima verossimilhança considerando $\alpha_{i}(i=1,2, \ldots, n)$ iguais à $\alpha$, portanto um modelo de efeitos fixos que considera nula a correlação entre as repetições. Também introduzimos uma análise Bayesiana onde são utilizados métodos de simulação de amostras baseados no método de Monte Carlo em Cadeias de Markov, considerando efeitos fixos, e efeitos aleatórios (1.5), com uma distribuição normal para $\alpha_{i}, i=1,2, \ldots, n$ e com uma mistura de distribuições normais.

Considerar,

$J$ : número de tempos;

n: número de observaçōes ;

$Y_{i j}(1$ ou 0$):$ a resposta para $i$-ésima observação $(i=1,2, \ldots, n)$ no $j$-ésimo tempo $(j=$ $1,2, \ldots, J)$, com distribuição de Bernoulli com parâmetro $\theta_{i j}$.

$x_{i j}=\left(x_{i j 1}, x_{i j 2}, \ldots, x_{i j p_{j}}\right):$ um vetor linha de regressão de dimensão $p_{j}$

A tabela a seguir apresenta a disposição dos dados binários em uma forma geral. 
Tabela 2.1: Dados Binários Longitudinais

\begin{tabular}{cccccc}
\hline$i$ & $T_{1}$ & $\cdots$ & $T_{j}$ & $\cdots$ & $T_{J}$ \\
\hline 1 & $y_{11} ;\left(x_{111}, x_{112}, \ldots, x_{11 p_{j}}\right)$ & $\cdots$ & $y_{1 j} ;\left(x_{1 j 1}, x_{1 j 2}, \ldots, x_{1 j p_{j}}\right)$ & $\cdots$ & $y_{1 J} ;\left(x_{1 J 1}, x_{1 J 2}, \ldots, x_{1 J_{p_{J}}}\right)$ \\
2 & $y_{21} ;\left(x_{211}, x_{212}, \ldots, x_{21 p_{j}}\right)$ & $\ldots$ & $y_{2 j} ;\left(x_{2 j 1}, x_{2 j 2}, \ldots, x_{2 j p_{j}}\right)$ & $\cdots$ & $y_{2 J} ;\left(x_{2 J 1}, x_{2 J 2}, \ldots, x_{2 J p_{J}}\right)$ \\
$\vdots$ & $\vdots$ & $\vdots$ & $\vdots$ & $\vdots$ & $\vdots$ \\
$i$ & $y_{i 1} ;\left(x_{i 11}, x_{i 12}, \ldots, x_{i 1 p_{j}}\right)$ & $\cdots$ & $y_{i j i}\left(x_{i j 1}, x_{i j 2}, \ldots, x_{i j p_{j}}\right)$ & $\cdots$ & $y_{i J} ;\left(x_{i J 1}, x_{i J 2}, \ldots, x_{i J_{j} J}\right)$ \\
$\vdots$ & $\vdots$ & $\vdots$ & $\vdots$ & $\vdots$ & $\vdots$ \\
$n$ & $y_{n 1} ;\left(x_{n 11}, x_{n 12}, \ldots, x_{n p_{j}}\right)$ & $\cdots$ & $y_{n j} ;\left(x_{n j 1}, x_{n j 2}, \ldots, x_{n j p_{j}}\right)$ & $\cdots$ & $y_{n J} ;\left(x_{n J 1}, x_{n J 2}, \ldots, x_{n j_{j}}\right)$ \\
\hline
\end{tabular}

O modelo proposto por Prentice (1988) é dado por

$$
\begin{array}{r}
\theta_{i j}=\frac{\exp \left\{\alpha_{i}+x_{i j} \beta_{j}\right\}}{1+\exp \left\{\alpha_{i}+x_{i j} \beta_{j}\right\}} \\
i=1,2, \ldots, n ; \quad j=1,2, \ldots J .
\end{array}
$$

Associado às covariáveis, temos $\beta_{j}=\left(\beta_{1 j}, \beta_{2 j}, \ldots, \beta_{p_{j} j}\right)^{\prime}$ um vetor coluna de coeficientes de regressão com dimensão $p_{j}$.

Tratando-se de uma resposta binária, $y_{i j}=1$ ou $y_{i j}=0$, com distribuição de Bernoulli de parâmetro $\theta_{i j}$, ou seja,

$$
\begin{array}{r}
\operatorname{Pr}\left[Y_{i j}=y_{i j} \mid \alpha_{i}, \beta_{j}, x_{i j}\right]=\theta_{i j}^{y_{i j}}\left(1-\theta_{i j}\right)^{1-y_{i j}} \\
i=1,2, \ldots, n ; j=1,2, \ldots J
\end{array}
$$

Substituindo a função de ligação (2.1) em (2.2), temos:

$$
\begin{array}{r}
\operatorname{Pr}\left[Y_{i j}=y_{i j} \mid \alpha_{i}, \beta_{j}, x_{i j}\right]=\frac{\exp \left\{\left(\alpha_{i}+x_{i j} \beta_{j}\right) y_{i j}\right\}}{1+\exp \left\{\alpha_{i}+x_{i j} \beta_{j}\right\}} \\
i=1,2, \ldots, n ; j=1,2, \ldots J .
\end{array}
$$

\subsection{Função de Verossimilhança}

Toda informação contida nos dados sobre os parâmetros do modelo é expressa pela função de verossimilhança (FV).

Assumindo que as observaçōes são independentes, a função de verossimilhança é obtida como o produto dos termos dados pela expressão (2.2), ou seja, o produto da probabilidade de ocorrência de cada resposta observada, podendo ser escrita na forma,

$$
\left.\mathrm{L} \underset{\sim}{\alpha}, \beta_{1}, \beta_{2}, \ldots, \beta_{J} \mid \mathcal{D}\right)=\prod_{i=1}^{n} \prod_{j=1}^{J} \theta_{i j}^{y_{i j}}\left(1-\theta_{i j}\right)^{1-y_{i j}}
$$


onde $\underset{\sim}{\alpha}=\left(\alpha_{1}, \alpha_{2}, \ldots, \alpha_{n}\right)$, e $\mathcal{D}$ representa o conjunto de dados $\left(y_{i j}, x_{i j}\right) i=1,2, \ldots, n$; $j=1,2, \ldots J$. Portanto, de acordo com a expressão (2.1) a funçāo de verossimilhança é dada por,

$$
\mathrm{L}\left(\underset{\sim}{\alpha}, \beta_{1}, \beta_{2}, \ldots, \beta_{J} \mid \mathcal{D}\right)=\prod_{i=1}^{n} \prod_{j=1}^{J} \frac{\exp \left\{\left(\alpha_{i}+x_{i j} \beta_{j}\right) y_{i j}\right\}}{1+\exp \left\{\alpha_{i}+x_{i j} \beta_{j}\right\}}
$$

\subsection{Análise Clássica do Modelo}

Inicialmente, consideramos efeitos fixos, ou seja, $\alpha_{i}=\alpha(i=1,2, \ldots, n)$. Para fazer as inferências sobre os parâmetros do modelo usamos o método da máxima verossimilhança, como é feito usualmente para parâmetros de modelos logísticos.

O objetivo é estimar os parâmetros $\alpha$ e $\beta_{j}, j=1,2, \ldots, J$, que maximizem a função de verossimilhança. Para isso tomamos o logaritmo da funçāo de verossimilhança (2.5), para simplificação matemática. A expressāo do logaritmo da função de verossimilhança é dado por,

$$
\begin{aligned}
\ell\left(\alpha, \beta_{1}, \beta_{2}, \ldots, \beta_{J} \mid \mathcal{D}\right) & =\log \mathrm{L}\left(\alpha, \beta_{1}, \beta_{2}, \ldots, \beta_{J} \mid \mathcal{D}\right) \\
& =\sum_{i=1}^{n} \sum_{j=1}^{J}\left\{\left(\alpha+x_{i j} \beta_{j}\right) y_{i j}-\log \left[1+\exp \left(\alpha+x_{i j} \beta_{j}\right)\right]\right\} \\
& =\sum_{i=1}^{n} \sum_{j=1}^{J}\left\{\alpha y_{i j}+x_{i j} \beta_{j} y_{i j}-\log \left[1+\exp \left(\alpha+x_{i j} \beta_{j}\right)\right]\right\} \\
& =\sum_{i=1}^{n} \sum_{j=1}^{J} \alpha y_{i j}+\sum_{i=1}^{n} \sum_{j=1}^{J} x_{i j} \beta_{j} y_{i j}-\sum_{i=1}^{n} \sum_{j=1}^{J} \log \left[1+\exp \left(\alpha+x_{i j} \beta_{j}\right)\right] .
\end{aligned}
$$

Derivando o logaritmo da função verossimilhança em relação à $\alpha$ e $\beta_{j}$, 
$(j=1,2, \ldots, J)$, temos:

$$
\begin{aligned}
\frac{\partial \ell}{\partial \alpha} & =\sum_{i=1}^{n} \sum_{j=1}^{J} y_{i j}-\sum_{i=1}^{n} \sum_{j=1}^{J} \frac{\exp \left\{\left(\alpha+x_{i j} \beta_{j}\right)\right\}}{1+\exp \left\{\alpha+x_{i j} \beta_{j}\right\}}=0 \\
\frac{\partial \ell}{\partial \beta_{p_{j} j}} & =\sum_{i=1}^{n} x_{i j} y_{i j}-\sum_{i=1}^{n} x_{i j p_{j}} \frac{\exp \left\{\left(\alpha+x_{i j} \beta_{j}\right)\right\}}{1+\exp \left\{\alpha+x_{i j} \beta_{j}\right\}}=0 \\
j & =1,2, \ldots, J .
\end{aligned}
$$

Igualando as equações (2.7) à zero, temos:

$$
\begin{aligned}
& \sum_{i=1}^{n} \sum_{j=1}^{J} y_{i j}-\sum_{i=1}^{n} \sum_{j=1}^{J} \frac{\exp \left\{\left(\widehat{\alpha}+x_{i j} \widehat{\beta}_{j}\right)\right\}}{1+\exp \left\{\widehat{\alpha}+x_{i j} \widehat{\beta}_{j}\right\}}=0 \\
& \sum_{i=1}^{n} x_{i j} y_{i j}-\sum_{i=1}^{n} x_{i j p_{j}} \frac{\exp \left\{\left(\widehat{\alpha}+x_{i j} \widehat{\beta}_{j}\right)\right\}}{1+\exp \left\{\widehat{\alpha}+x_{i j} \widehat{\beta}_{j}\right\}}=0 \\
& j=1,2, \ldots, J
\end{aligned}
$$

As expressões (2.8) são as funções Escore. Os valores de $\alpha$ e $\beta_{j}$ que são soluções das equaçōes (2.8) serão os EMV denotados por $\widehat{\alpha}$ e $\widehat{\beta}_{j}$. Um método prático para obtenção das soluçōes é o método de Newton-Raphson, que é um algoritmo numérico, que coincide com o proposto por Fisher (Rao, 1973). As estimativas de máxima verossimilhança sāo obtidas através do seguinte esquema iterativo:

$$
\left(\begin{array}{l}
\widehat{\alpha}_{(n+1)} \\
\widehat{\beta}_{j(n+1)}
\end{array}\right)=\left(\begin{array}{c}
\widehat{\alpha}_{(n)} \\
\widehat{\beta}_{j(n)}
\end{array}\right)+\left(\mathcal{I}\left(\widehat{\alpha}_{(n)}, \widehat{\beta}_{j(n)}\right)\right)^{-1} S\left(\widehat{\alpha}_{(n)}, \widehat{\beta}_{j(n)}\right)
$$

onde $\mathcal{I}$ é a matriz de informação de Fisher e $S$ o vetor Escore. 
As segundas derivadas do logaritmo da função de verossimilhança são dadas por:

$$
\begin{aligned}
& \frac{\partial^{2} \ell}{\partial \alpha^{2}}=-\sum_{i=1}^{n} \sum_{j=1}^{J} \frac{\exp \left\{\left(\alpha+x_{i j} \beta_{j}\right)\right\}}{\left[1+\exp \left(\alpha+x_{i j} \beta_{j}\right)\right]^{2}} \\
& \frac{\partial^{2} \ell}{\partial \beta_{p_{j} j}^{2}}=-\sum_{i=1}^{n} x_{i j p_{j}}^{2} \frac{\exp \left\{\left(\alpha+x_{i j} \beta_{j}\right)\right\}}{\left[1+\exp \left(\alpha+x_{i j} \beta_{j}\right)\right]^{2}} \\
& \frac{\partial^{2} \ell}{\partial \alpha \partial \beta_{p_{j} j}}=-\sum_{i=1}^{n} x_{i j p_{j}} \frac{\exp \left\{\left(\alpha+x_{i j} \beta_{j}\right)\right\}}{\left[1+\exp \left(\alpha+x_{i j} \beta_{j}\right)\right]^{2}} \\
& \frac{\partial^{2} \ell}{\partial \beta_{p_{j} j} \partial \beta_{p_{k} j}}=0 \text { para } j \neq k \text { onde }\left\{\begin{array}{l}
j=1,2, \ldots, J \\
k=1,2, \ldots, J
\end{array}\right. \\
& \begin{cases}\frac{\partial^{2} \ell}{\partial \beta_{p_{j} j} \partial \beta_{p_{k} k}}=0 & \text { para } \\
\frac{\partial^{2} \ell}{\partial \beta_{p_{j} j} \partial \beta_{p_{k} k}}=-\sum_{i=1}^{n} x_{i j p_{j}} x_{i j p_{k}} \frac{\exp \left\{\left(\alpha+x_{i j} \beta_{j}\right)\right\}}{\left[1+\exp \left(\alpha+x_{i j} \beta_{j}\right)\right]^{2}} & \text { c.c. }\end{cases}
\end{aligned}
$$

Os estimadores de máxima verossimilhança têm distribuição normal assintótica (ver por exemplo, Gourieroux \& Monfort, 1995; Bickel \& Doksum, 1977), ou seja:

$$
\left(\widehat{\alpha}, \widehat{\beta}_{1}, \widehat{\beta}_{2}, \ldots, \widehat{\beta}_{J}\right) \stackrel{a}{\sim} N\left[\left(\alpha, \beta_{1}, \beta_{2}, \ldots, \beta_{J}\right), \mathcal{I}^{-1}\right]
$$

onde $\mathcal{I}$ é a matriz de informação de Fisher.

No passo final das iterações além de obtermos uma estimativa dos parâmetros temos uma estimativa da matriz de variância-covariância dos estimadores.

Este processo pode ser árduo se tivermos um número de parâmetros muito grande para serem estimados. Para usar este método de resoluçāo iterativa temos que dar valores adequados muito aproximados dos valores dos parâmetros ou chutes iniciais, a fim de obter convergência para os pontos $\hat{\alpha}$ e $\widehat{\beta}_{j}$ com poucas iterações. Uma forma de obtermos os chutes iniciais é fazer uma aproximação do logaritmo da verossimilh ança (ver por exemplo, 
Cox, 1970) como a seguir:

$$
\ell\left(\alpha, \beta_{1}, \beta_{2}, \ldots, \beta_{J} \mid \mathcal{D}\right)=\sum_{i=1}^{n} \sum_{j=1}^{J} \alpha y_{i j}+\sum_{i=1}^{n} \sum_{j=1}^{J} x_{i j} \beta_{j} y_{i j}-K,
$$

onde

$$
K=\sum_{i=1}^{n} \sum_{j=1}^{J} \log \left[1+\exp \left(\alpha+x_{i j} \beta_{j}\right)\right]
$$

Considerando os resultados,

- $\log (1+x) \simeq x-\frac{x^{2}}{2}+\frac{x^{3}}{3}-\cdots+\frac{(-1)^{n} x^{n+1}}{n+1}+\cdots$

- $e^{x} \simeq 1+x+\frac{x^{2}}{2 !}+\cdots+\frac{x^{n}}{n !}+\cdots$

podemos escrever,

$$
K \simeq e^{\alpha} \sum_{i=1}^{n} \sum_{j=1}^{J}\left[1+x_{i j} \beta_{j}+\frac{1}{2}\left(x_{i j} \beta_{j}\right)^{2}\right]
$$

Assim o logaritmo da função de verossimilhança aproximada é dado por,

$\ell^{*}\left(\alpha, \beta_{1}, \beta_{2}, \ldots, \beta_{J} \mid \mathcal{D}\right)=\sum_{i=1}^{n} \sum_{j=1}^{J} \alpha y_{i j}+\sum_{i=1}^{n} \sum_{j=1}^{J} x_{i j} \beta_{j} y_{i j}-e^{\alpha} \sum_{i=1}^{n} \sum_{j=1}^{J}\left[1+x_{i j} \beta_{j}+\frac{1}{2}\left(x_{i j} \beta_{j}\right)^{2}\right]$

Derivando $\ell^{*}\left(\alpha, \beta_{1}, \beta_{2}, \ldots, \beta_{J} \mid \mathcal{D}\right)$ nos parâmetros e igualando a zero, obtemos facilmente as estimativas aproximadas dos EMV's dos parâmetros que servirão de chutes iniciais para o método de Newton-Raphson.

\subsection{Análise Bayesiana do Modelo}

Modelo 1: Modelo logístico com efeitos fixos.

Nesta seção consideramos o uso de métodos Bayesianos para o modelo de Prentice (1988). Para isso, usamos métodos MCMC em particular o algoritmo Gibbs Sampling (ver, Gelfand, A.E. \& Smith, A.F.M, 1990) e o algoritmo Metropolis-Hastings (ver, Chib, S. \& Greenberg, E., 1995), os quais tem sido muito utilizados em análise Bayesiana.

Ambos os métodos baseiam-se nas distribuições condicionais a posteriori para geração dos parâmetros, sendo o algoritmo Gibbs Sampling usado quando a distribuição condicional possui uma forma padronizada conhecida e o algoritmo Metropolis-Hastings usado quando a distribuição condicional não tem uma forma conhecida. 
Assumimos as seguintes densidades a priori informativas:

(i) $\alpha \sim N\left(\mu_{0}, \sigma_{0}^{2}\right)$ com $\mu_{0}$ e $\sigma_{0}^{2}$ conhecidos;

(ii) $\beta_{k j} \sim N\left(b_{k j}, c_{k j}^{2}\right)$ com $b_{k j}$ e $c_{k j}^{2}$ conhecidos;

$$
j=1,2, \ldots, J ; k=1,2, \ldots, p_{j} .
$$

Assumindo independência das distribuiçōes a priori, a distribuiçāo a priori conjunta é dada por,

$$
\pi\left(\alpha, \beta_{1}, \beta_{2}, \ldots, \beta_{J}\right) \propto \exp \left\{-\frac{\left(\alpha-\mu_{0}\right)^{2}}{2 \sigma_{0}^{2}}\right\} \prod_{j=1}^{J} \prod_{k=1}^{p_{j}} \exp \left\{-\frac{\left(\beta_{k j}-b_{k j}\right)^{2}}{2 c_{k j}^{2}}\right\}
$$

A densidade a posteriori conjunta é tal que,

$$
\pi\left(\alpha, \beta_{1}, \beta_{2}, \ldots, \beta_{J} \mid \mathcal{D}\right) \propto \pi\left(\alpha, \beta_{1}, \beta_{2}, \ldots, \beta_{J}\right) \mathrm{E}\left(\alpha, \beta_{1}, \beta_{2}, \ldots, \beta_{J} \mid \mathcal{D}\right)
$$

Desta forma, temos,

$$
\begin{aligned}
\pi\left(\alpha, \beta_{1}, \beta_{2}, \ldots, \beta_{J} \mid \mathcal{D}\right) \propto & {\left[\exp \left\{-\frac{\left(\alpha-\mu_{0}\right)^{2}}{2 \sigma_{0}^{2}}\right\} \prod_{j=1}^{J} \prod_{k=1}^{p_{j}} \exp \left\{-\frac{\left(\beta_{k j}-b_{k j}\right)^{2}}{2 c_{k j}^{2}}\right\}\right] } \\
& \cdot\left[\prod_{i=1}^{n} \prod_{j=1}^{J} \frac{\exp \left\{\left(\alpha+x_{i j} \beta_{j}\right) y_{i j}\right\}}{1+\exp \left\{\alpha+x_{i j} \beta_{j}\right\}}\right] .
\end{aligned}
$$

Para obtermos uma amostra da densidade a posteriori marginal dos parâmetros $\left(\alpha, \beta_{1}, \beta_{2}, \ldots, \beta_{J}\right)$ com o uso do método de Monțe Carlo em Cadeias de Markov, temos as seguintes distribuições condicionais a posteriori:

(i)

$$
\begin{aligned}
& \pi\left(\alpha \mid \beta_{1}, \beta_{2}, \ldots, \beta_{J}, \mathcal{D}\right) \propto \exp \left\{-\frac{\left(\alpha-\mu_{0}\right)^{2}}{2 \sigma_{0}^{2}}\right\} \prod_{i=1}^{n} \prod_{j=1}^{J} \frac{\exp \left\{\left(\alpha+x_{i j} \beta_{j}\right) y_{i j}\right\}}{1+\exp \left\{\alpha+x_{i j} \beta_{j}\right\}}, \quad \text { ou } \\
& \pi\left(\alpha \mid \beta_{1}, \beta_{2}, \ldots, \beta_{J}, \mathcal{D}\right) \propto N\left(\mu_{0}, \sigma_{0}^{2}\right) \cdot \Psi_{1}\left(\alpha, \beta_{1}, \beta_{2}, \ldots, \beta_{J}\right)
\end{aligned}
$$


onde

$$
\begin{aligned}
& \quad \Psi_{1}\left(\alpha, \beta_{1}, \beta_{2}, \ldots, \beta_{J}\right)=\exp \left\{\alpha y . .-\sum_{i=1}^{n} \sum_{j=1}^{J} \log \left[1+\exp \left(\alpha+\sum_{l=1}^{p_{j}} x_{i j l} \beta_{l j}\right)\right]\right\} \\
& y . .=\sum_{i=1}^{n} \sum_{j=1}^{J} y_{i j} .
\end{aligned}
$$

(ii)

$$
\begin{aligned}
& \pi\left(\beta_{k l} \mid \alpha, \beta_{1}, \ldots, \beta_{j-1}, \underset{\sim}{\beta}, \beta_{j+1}, \ldots, \beta_{J}, \mathcal{D}\right) \propto \\
& \propto \exp \left\{-\frac{\left(\beta_{k l}-b_{k l}\right)^{2}}{2 c_{k l}^{2}}\right\} \prod_{i=1}^{n} \frac{\exp \left\{\left(\alpha+x_{i j} \beta_{j}\right) y_{i j}\right\}}{1+\exp \left\{\alpha+x_{i j} \beta_{j}\right\}} \\
& \propto N\left(b_{k l}, c_{k l}^{2}\right) \cdot \Psi_{2}\left(\alpha, \beta_{1}, \beta_{2} \ldots, \beta_{J}, \mathcal{D}\right)
\end{aligned}
$$

onde

$$
\Psi_{2}\left(\alpha, \beta_{1}, \beta_{2} \ldots, \beta_{J}, \mathcal{D}\right)=\exp \left\{a_{k l} \beta_{k l}-\sum_{i=1}^{n} \log \left[1+\exp \left(\alpha+x_{i j} \beta_{j}\right)\right]\right\}
$$

$a_{k l}=\sum_{i=1}^{n} x_{i l k} y_{i l} ; \quad k=1, \ldots, p_{j} ; \quad l, j=1, \ldots, J$

e $\underset{\sim}{\beta}$ é um vetor de dimensão $p_{l}-1$, ou seja:

$$
\stackrel{\beta}{\sim}_{k l}= \begin{cases}\left(\beta_{2 l}, \beta_{3 l}, \ldots, \beta_{p_{l}}\right) & \text { se } k=1 \\ \left(\beta_{1 l}, \ldots, \beta_{k-1, l}, \beta_{k+1, l}, \ldots, \beta_{p_{l} l}\right) & \text { se } 2 \leq k \leq p_{l-1} \\ \left(\beta_{1 l}, \ldots, \beta_{p_{l-1}, l}\right) & \text { se } k=p_{l}\end{cases}
$$

$$
\left\{\begin{array}{l}
\mathrm{e} \\
l=1,2, \ldots, J \\
k=1,2, \ldots, p_{j}
\end{array}\right.
$$

Como podemos observar, nenhuma das distribuições condicionais são padronizadas, portanto devemos usar o algoritmo de Metropolis-Hastings para simulação dos parâmetros, 
e a partir dos valores simulados obter a média, variância e outras inferências a posteriori de interesse.

Para o uso algoritmo de Metropolis-Hastings consideramos a parte conhecida (padronizada) das distribuições condicionais a posteriori como núcleo de transição do algoritmo. Para verificar a convergência da amostra simulada de cada parâmetro para uma distribuiçāo estabilizada utilizamos o algoritmo proposto por Gelman e Rubin (1992).

\subsection{Análise Bayesiana Hierárquica Assumindo Efeitos Aleatórios Normais}

Modelo 2: Modelo logístico assumindo efeitos aleatórios normais $-\alpha_{i} \stackrel{i i d}{\sim} N\left(0, \sigma_{\alpha}^{2}\right)$;

$$
i=1, \ldots, n
$$

Assumir agora que $\alpha_{i},(i=1, \ldots, n)$ são efeitos aleatórios independentes com distribuição normal, isto é,

$$
\alpha_{i} \sim N\left(0, \sigma_{\alpha}^{2}\right)
$$

onde $\sigma_{\alpha}^{2}$ é desconhecido com uma distribuição conhecida, portanto $\sigma_{\alpha}^{2}$ é um hiperparâmetro e $N\left(0, \sigma_{\alpha}^{2}\right)$ uma distribuição a priori hierárquica para $\alpha_{i}, \quad(i=1, \ldots, n)$, sendo assim necessário a atribuição de uma distribuição a priori para este hiperparâmetro.

Consideramos as seguintes distribuiçōes a priori para $\sigma_{\alpha}^{2}$ e $\beta_{l j}, \quad l=1, \ldots, p_{j}, \quad j=$ $1, \ldots, J$.

(i) $\sigma_{\alpha}^{2} \sim \mathcal{I G}(a, b) \quad$ com $a$ e $b$ conhecidos;

(ii) $\beta_{l j} \sim N\left(b_{l j}, c_{l j}^{2}\right)$ com $b_{l j}$ e $c_{l j}^{2}$ conhecidos,

$$
l=1, \ldots, p_{j}, \quad j=1, \ldots, J
$$

onde $\mathcal{I G}$ é uma distribuição Gama Invertida,

$$
\pi\left(\sigma_{\alpha}^{2}\right)=\frac{1}{\Gamma(a)} b^{-a}\left(\sigma_{\alpha}^{2}\right)^{-(a+1)} \exp \left\{-\frac{1}{b \sigma_{\alpha}^{2}}\right\} .
$$

Isto é,

$$
\pi\left(\sigma_{\alpha}^{2}\right) \propto\left(\sigma_{\alpha}^{2}\right)^{-(a+1)} \exp \left\{-\frac{1}{b \sigma_{\alpha}^{2}}\right\}
$$


para $\sigma_{\alpha}^{2}>0$ com média $\frac{1}{b(a-1)}$ para $a>1$ e variância $\frac{1}{b^{2}(a-1)^{2}(a-2)}$ para $a>2$.

Assumir independência a priori para os parâmetros. A distribuição a posteriori conjunta (a partir de (2.5), (2.13) e (2.14)) para $\underset{\sim}{\alpha}=\left(\alpha_{1}, \ldots, \alpha_{n}\right), \quad \sigma_{\alpha}^{2}$ e $\beta_{1}, \ldots, \beta_{J}$ é dada por,

$$
\begin{aligned}
\pi\left(\underset{\sim}{\alpha}, \sigma_{\alpha}^{2}, \beta_{1}, \ldots, \beta_{J} \mid \mathcal{D}\right) \propto & \\
\propto & \left(\sigma_{\alpha}^{2}\right)^{-\left(\frac{n}{2}+a+1\right)} \exp \left\{-\frac{1}{2 \sigma_{\alpha}^{2}}\left[\frac{2}{b}+\sum_{i=1}^{n} \alpha_{i}^{2}\right]\right\} \\
& \cdot\left[\prod_{l=1}^{p_{j}} \prod_{j=1}^{J} \exp \left\{-\frac{1}{2 c_{l j}^{2}}\left(\beta_{l j}-b_{l j}\right)^{2}\right\}\right] \\
& \cdot\left[\prod_{i=1}^{n} \prod_{j=1}^{J} \frac{\exp \left\{\left(\alpha_{i}+x_{i j} \beta_{j}\right) y_{i j}\right\}}{1+\exp \left\{\alpha_{i}+x_{i j} \beta_{j}\right\}}\right]
\end{aligned}
$$

As distribuições condicionais necessárias para o algoritmo Gibbs sampling são dadas por,

i)

$$
\pi\left(\alpha_{i} \mid \sigma_{\alpha}^{2}, \beta_{1}, \ldots, \beta_{J}, \mathcal{D}\right) \propto \exp \left\{-\frac{\alpha_{i}^{2}}{2 \sigma_{\alpha}^{2}}\right\} \frac{\exp \left\{\alpha_{i} y_{i .}\right\}}{\prod_{j=1}^{J}\left\{1+\exp \left(\alpha_{i}+x_{i j} \beta_{j}\right)\right\}}
$$

para $i=1, \ldots, n$, isto é,

$$
\pi\left(\alpha_{i} \mid \sigma_{\alpha}^{2}, \beta_{1}, \ldots, \beta_{J}, \mathcal{D}\right) \propto N\left\{0, \sigma_{\alpha}^{2}\right\} \Psi_{3}\left(\alpha_{i}, \beta_{1}, \ldots, \beta_{J}\right)
$$

onde

$$
\Psi_{3}\left(\alpha_{i}, \beta_{1}, \ldots, \beta_{J}\right)=\exp \left\{\alpha_{i} \sum_{j=1}^{J} y_{i j}-\sum_{j=1}^{J} \log \left[1+\exp \left(\alpha_{i}+x_{i j} \beta_{j}\right)\right]\right\}
$$

ii)

$$
\begin{aligned}
\pi\left(\sigma_{\alpha}^{2} \mid \underset{\sim}{\alpha}, \beta_{1}, \ldots, \beta_{J}, \mathcal{D}\right) & \propto\left(\sigma_{\alpha}^{2}\right)^{-\left(\frac{n}{2}+a+1\right)} \exp \left\{-\frac{1}{\sigma_{\alpha}^{2}}\left[\frac{1}{b}+\frac{1}{2} \sum_{i=1}^{n} \alpha_{i}^{2}\right]\right\} \\
& \propto\left(\sigma_{\alpha}^{2}\right)^{-\left(\frac{\pi}{2}+a+1\right)} \exp \left\{-\frac{1}{\sigma_{\alpha}^{2}\left[\frac{1}{b}+\frac{1}{2} \sum_{i=1}^{n} \alpha_{i}^{2}\right]^{-1}}\right\}
\end{aligned}
$$

isto é,

$$
\sigma_{\alpha}^{2} \mid \underset{\sim}{\alpha}, \beta_{1}, \ldots, \beta_{J}, \mathcal{D} \sim \mathcal{I} \mathcal{G}\left[\frac{n}{2}+a ;\left(\frac{1}{b}+\frac{1}{2} \sum_{i=1}^{n} \alpha_{i}^{2}\right)^{-1}\right]
$$


iii)

$$
\pi\left(\beta_{k l} \mid \underset{\sim}{\alpha}, \beta_{1}, \ldots, \beta_{j-1}, \underset{\sim}{\beta}, \beta_{j l}, \ldots, \beta_{J}, \mathcal{D}\right) \propto N\left(b_{k l}, c_{k l}^{2}\right) \frac{\exp \left\{\beta_{k l} a_{k l}\right\}}{\prod_{i=1}^{n}\left\{1+\exp \left(\alpha_{i}+x_{i j} \beta_{j}\right)\right\}}
$$

isto é,

$$
\pi\left(\beta_{k l} \mid \underset{\sim}{\alpha}, \beta_{1}, \ldots, \beta_{j-1}, \underset{\sim}{\beta}, \beta_{j+1}, \ldots, \beta_{J}, \mathcal{D}\right) \propto N\left(b_{k l}, c_{k l}^{2}\right) \Psi_{4}\left(\underset{\sim}{\alpha}, \beta_{1}, \ldots, \beta_{J}\right)
$$

onde

$$
\Psi_{4}\left(\underset{\sim}{\alpha}, \beta_{1}, \ldots, \beta_{J}\right)=\exp \left\{\beta_{k l} a_{k l}-\sum_{j=1}^{n} \log \left[1+\exp \left(\alpha_{i}+x_{i j} \beta_{j}\right)\right]\right\}
$$

$, a_{k l}=\sum_{i=1}^{n} x_{i l k} y_{i l}, \quad k=1, \ldots, p_{j}, . \quad l, j=1, \ldots, J, \underset{\sim}{\beta}$ definido em (2.12).

Observar que $\alpha_{i}$ e $\beta_{k l}$ devem ser gerados a partir do algoritmo Metropolis-Hastings.

\subsection{Modelo de Efeitos Aleatórios com uma Mistura de Distribuições Normais}

Modelo 3: Modelo logístico assumindo os efeitos aleatórios com uma mistura de distribuiçōes Normais.

Quando assumimos $\alpha_{i}, i=1, \ldots, n$ como efeitos aleatórios independentes, podemos ter uma multimodalidade e portanto modelar estes parâmetros por uma única distribuição normal pode não ser apropriado. Sendo assim para maior flexibilidade, e consistência com a estratégia de modelagem hierárquica, construímos tais distribuições como misturas de distribuições normais.

Procurando construir um modelo mais realista assumimos para $\alpha_{i}(i=1, \ldots, n)$ uma mistura de duas distribuições normais, com densidade,

$$
\begin{aligned}
\pi\left(\alpha_{i}\right) & =\sum_{k=1}^{2} p_{k} \phi_{k}\left(\alpha_{i} \mid \mu_{k}, \sigma_{k}^{2}\right) \\
& =p_{1} \phi_{1}\left(\alpha_{i} \mid \mu_{1}, \sigma_{1}^{2}\right)+\left(1-p_{1}\right) \phi_{2}\left(\alpha_{i} \mid \mu_{2}, \sigma_{2}^{2}\right)
\end{aligned}
$$

onde $\phi_{k}$ denota uma densidade normal $N\left(\mu_{k}, \sigma_{k}^{2}\right)$, com a restrição $p_{1}+p_{2}=1$. 
Assumimos as seguintes distribuições a priori para $\mu_{k}, \sigma_{k}^{2}, \beta_{l j}$ e $p_{1}$ :

(i) $\mu_{k} \sim N\left(d_{k}, e_{k}^{2}\right) \quad$ com $d_{k}$ e $e_{k}^{2}$ conhecidos, $k=1,2$;

(ii) $\sigma_{k}^{2} \sim \mathcal{I} \mathcal{G}\left(a_{k}, b_{k}\right) \quad$ com $a_{k}$ e $b_{k}$ conhecidos, $k=1,2$;

(iii) $\beta_{l j} \sim N\left(b_{l j}, c_{l j}^{2}\right)$ com $b_{l j}$ e $c_{l j}^{2}$ conhecidos, $l=1, \ldots, p_{j} ; j=1, \ldots, J$

(iv) $p_{1} \sim \mathcal{B}(f, g)$ com $f$ e $g$ conhecidos

onde $\mathcal{B}(f, g)$ denota uma distribuição beta com densidade

$$
\pi\left(p_{1}\right) \propto p_{1}^{f-1}\left(1-p_{1}\right)^{g-1}, \quad 0<p_{1}<1
$$

Assumir independência a priori entre os parâmetros. A distribuição a posteriori conjunta para $\underset{\sim}{\alpha}=\left(\alpha_{1}, \ldots, \alpha_{n}\right), \underset{\sim}{\mu}=\left(\mu_{1}, \mu_{2}\right), p_{1}$ e $\underset{\sim}{\sigma^{2}}=\left(\sigma_{1}^{2}, \sigma_{2}^{2}\right)$ é dada por,

$$
\begin{aligned}
\left.\pi \underset{\sim}{\alpha}, \beta_{1}, \ldots, \beta_{J}, \underset{\sim}{\mu},{\underset{\sim}{\sim}}^{2}, p_{1} \mid \mathcal{D}\right) \propto & \prod_{i=1}^{n}\left\{\sum_{k=1}^{2} p_{k} \phi_{k}\left(\alpha_{i} \mid \mu_{k}, \sigma_{k}^{2}\right)\right\} \cdot\left\{\prod_{k=1}^{2}\left(\sigma_{k}^{2}\right)^{-\left(a_{k}+1\right)} \exp \left[\frac{-1}{b_{k} \sigma_{k}^{2}}\right]\right\} \\
& \cdot\left\{\prod_{k=1}^{2} \exp \left[-\frac{1}{2 e_{k}^{2}}\left(\mu_{k}-d_{k}\right)^{2}\right]\right\} \cdot p_{1}^{f-1}\left(1-p_{1}\right)^{g-1} \\
& \cdot\left\{\prod_{l=1}^{p_{j}} \prod_{j=1}^{J} \exp \left[-\frac{1}{2 c_{l j}^{2}}\left(\beta_{l j}-b_{l j}\right)^{2}\right]\right\} \\
& \frac{\exp \left\{\sum_{i=1}^{n} \sum_{j=1}^{J}\left(\alpha_{i}+x_{i j} \beta_{j}\right) y_{i j}\right\}}{\prod_{i=1}^{n} \prod_{j=1}^{J}\left\{1+\exp \left(\alpha_{i}+x_{i j} \beta_{j}\right)\right\}}
\end{aligned}
$$

Como podemos observar, a mistura de distribuições normais envolve uma soma de distribuições dificultando o cálculo das distribuiçōes à posteriori marginais dos parâmetros do modelo, para uma análise Bayesiana. Para simplificar as distribuições condicionais necessárias para o algoritmo Gibbs Sampling, consideramos a introdução de variáveis latentes, que irá transformar o modelo de misturas em um modelo de componentes independentes (ver por exemplo, Tanner \& Wong, 1987), dados por $\underset{\sim}{z}=\left(z_{i 1}, z_{i 2}\right), z_{i 1}+z_{i 2}=1$, 
$i=1, \ldots, n$ onde $z_{i 1}$ tem uma distribuição de Bernoulli $b\left(1, h_{i 1}\right), \mathrm{e}$

$$
h_{i l}=\frac{p_{1} \phi_{1}\left(\alpha_{i} \mid \mu_{1}, \sigma_{1}^{2}\right)}{p_{1} \phi_{1}\left(\alpha_{i} \mid \mu_{1}, \sigma_{1}^{2}\right)+\left(1-p_{1}\right) \phi_{2}\left(\alpha_{i} \mid \mu_{2}, \sigma_{2}^{2}\right)}
$$

Isto é,

$$
\begin{aligned}
\pi\left({\underset{\sim}{1}}_{1}^{z}, \ldots, \underset{\sim_{n}}{z}\right) & \propto \prod_{i 1}^{n} h_{i 1}^{z_{i 1}}\left(1-h_{i 1}\right)^{z_{i 2}} \\
& \propto \frac{\prod_{i=1}^{n} \prod_{k=1}^{2}\left[p_{k} \phi_{k}\left(\alpha_{i} \mid \mu_{k}, \sigma_{k}^{2}\right)\right]^{z_{i k}}}{\prod_{i=1}\left\{\sum_{k=1}^{2} p_{k} \phi_{k}\left(\alpha_{i} \mid \mu_{k}, \sigma_{k}^{2}\right)\right\}}
\end{aligned}
$$

onde,

$$
z_{i 1}= \begin{cases}1 & \text { com probabilidade } h_{i 1}, \\ 0 & \text { com probabilidade } 1-h_{i 1} .\end{cases}
$$

Combinando-se (2.19) com (2.21), temos a seguinte densidade à posteriori conjunta para os parâmetros (sendo $\left.\underset{\sim}{z}=\left(\underset{\sim_{1}}{z}, \ldots, \underset{\sim_{n}}{z}\right)\right)$,

$$
\begin{aligned}
\pi\left(\underset{\sim}{\alpha}, \beta_{1}, \ldots, \beta_{J}, \underset{\sim}{\sim} \underset{\sim}{\left.\sigma^{2}, p_{1} \mid \underset{\sim}{z}, \mathcal{D}\right) \propto} \prod_{i=1}^{n} \prod_{k=1}^{2}\left[p_{k} \phi_{k}\left(\alpha_{i} \mid \mu_{k}, \sigma_{k}^{2}\right)\right]^{z_{i 2}}\right. \\
\cdot\left\{\prod_{k=1}^{2}\left(\sigma_{k}^{2}\right)^{-\left(a_{k}+1\right)} \exp \left[\frac{-1}{b_{k} \sigma_{k}^{2}}\right]\right\} \cdot p_{1}^{f-1}\left(1-p_{1}\right)^{g-1} \\
\cdot\left\{\prod_{k=1}^{2} \exp \left[-\frac{1}{2 e_{k}^{2}}\left(\mu_{k}-d_{k}\right)^{2}\right]\right\} \\
\cdot\left\{\prod_{l=1}^{p_{j}} \prod_{j=1}^{J} \exp \left[-\frac{1}{2 c_{l j}^{2}}\left(\beta_{l j}-b_{l j}\right)^{2}\right]\right\} \\
\cdot \\
\cdot \frac{\exp \left\{\sum_{i=1}^{n} \sum_{j=1}^{J}\left(\alpha_{i}+x_{i j} \beta_{j}\right) y_{i j}\right\}}{\prod_{i=1}^{n} \prod_{j=1}^{J}\left\{1+\exp \left(\alpha_{i}+x_{i j} \beta_{j}\right)\right\}}
\end{aligned}
$$


De (2.22), encontramos as distribuiçōes condicionais necessárias para o algoritmo Gibbs sampling,

(i)

$$
\pi\left(\alpha_{i} \mid \beta_{1}, \ldots, \beta_{J}, \underset{\sim}{\mu} \underset{\sim}{\sigma^{2}}, p_{1}, \underset{\sim}{z}, \mathcal{D}\right) \propto \frac{\phi_{1}^{z_{i 1}}\left(\alpha_{i} \mid \mu_{1}, \sigma_{1}^{2}\right) \phi_{2}^{z_{i 2}}\left(\alpha_{i} \mid \mu_{2}, \sigma_{2}^{2}\right) \exp \left(\alpha_{i} y_{i .}\right)}{\prod_{j=1}^{J}\left\{1+\exp \left(\alpha_{i}+x_{i j} \beta_{j}\right)\right\}}
$$

onde $y_{i}=\sum_{j=1}^{J} y_{i j}$.

Isto é,

$$
\begin{gathered}
\pi\left(\alpha_{i} \mid \beta_{1}, \ldots, \beta_{J} \underset{\sim}{\mu}, \stackrel{\sigma}{\sim}^{2}, p_{1}, \underset{\sim}{z}, \mathcal{D}\right) \propto \exp \left\{-\frac{\left(z_{i 1} \sigma_{2}^{2}+z_{i 2} \sigma_{1}^{2}\right)}{2 \sigma_{1}^{2} \sigma_{2}^{2}}\left[\alpha_{i}-\frac{z_{i 1} \mu_{1} \sigma_{2}^{2}+z_{i 2} \mu_{2} \sigma_{2}^{2}}{z_{i 1} \sigma_{2}^{2}+z_{i 2} \sigma_{1}^{2}}\right]^{2}\right\} \\
\cdot \frac{\exp \left\{\alpha_{i} y_{i}\right\}}{\prod_{j=1}^{J}\left\{1+\exp \left(\alpha_{i}+x_{i j} \beta_{j}\right)\right\}}
\end{gathered}
$$

ou,

$$
\begin{gathered}
\pi\left(\alpha_{i} \mid \beta_{1}, \ldots, \beta_{J}, \underset{\sim}{\sim} \underset{\sim}{\sigma^{2}}, p_{1}, \underset{\sim}{z}, \mathcal{D}\right) \propto N\left\{\frac{z_{i 1} \mu_{1} \sigma_{2}^{2}+z_{i 2} \mu_{2} \sigma_{1}^{2}}{z_{i 1} \sigma_{2}^{2}+z_{i 2} \sigma_{1}^{2}}, \frac{\sigma_{1}^{2} \sigma_{2}^{2}}{z_{i 1} \sigma_{2}^{2}+z_{i 2} \sigma_{1}^{2}}\right\} \\
\cdot \Psi_{5}\left(\alpha_{i}, \beta_{1}, \ldots, \beta_{J}\right),
\end{gathered}
$$

onde,

$$
\Psi_{5}\left(\alpha_{i}, \beta_{1}, \ldots, \beta_{J}\right)=\exp \left\{\alpha_{i} y_{i}-\sum_{j=1}^{J} \log \left[1+\exp \left(\alpha_{i}+x_{i j} \beta_{j}\right)\right]\right\}
$$

para $i=1, \ldots, n$.

(ii)

$$
\begin{array}{r}
\pi\left(\sigma_{k}^{2} \mid \alpha_{i}, \beta_{1}, \ldots, \beta_{J}, \underset{\sim}{\mu}, \sigma_{\underline{k}}^{2}, p_{1}, \underset{\sim}{z}, \mathcal{D}\right) \propto\left(\sigma_{k}^{2}\right)^{-\left(a_{k}+1\right)} \exp \left\{-\frac{1}{b_{k} \sigma_{k}^{2}}\right\}\left(\sigma_{k}^{2}\right)^{-\frac{v_{k}}{2}} \\
\cdot \exp \left\{-\frac{1}{2 \sigma_{k}^{2}} \sum_{i=1}^{n} z_{i k}\left(\alpha_{i}-\mu_{k}\right)^{2}\right\}
\end{array}
$$


onde $v_{k}=\sum_{i=1}^{n} z_{i k}$

Isto é,

$$
\sigma_{k}^{2} \mid \alpha_{i}, \beta_{1}, \ldots, \beta_{J}, \underset{\sim}{\mu}, p_{1}, \underset{\sim}{z}, \mathcal{D} \sim \mathcal{I} \mathcal{G}\left[a_{k}+\frac{v_{k}}{2} ;\left(\frac{1}{b_{k}}+\frac{\sum_{i=1}^{n} z_{i k}\left(\alpha_{i}-\mu_{k}\right)^{2}}{2}\right)^{-1}\right]
$$

onde $k \neq \underline{k}, \quad k, \underline{k}=1,2$

(iii)

$$
p_{1} \mid \underset{\sim}{\alpha}, \beta_{1}, \ldots, \beta_{J}, \underset{\sim}{\mu} \underset{\sim}{\sigma^{2}} \underset{\sim}{z}, \mathcal{D} \sim \mathcal{B}\left(f+v_{1}, g+v_{2}\right)
$$

(iv)

$\pi\left(\beta_{k l} \mid \underset{\sim}{\alpha}, \beta_{1}, \ldots, \beta_{j-1}, \underset{\sim}{\beta}, \beta_{j+1}, \ldots, \beta_{J}, \underset{\sim}{\mu} \underset{\sim}{\sigma^{2}}, p_{1}, \underset{\sim}{z}, \mathcal{D}\right) \propto N\left(b_{k l}, c_{k l}^{2}\right) \Psi_{6}\left(\underset{\sim}{\alpha}, \beta_{1}, \ldots, \beta_{J}\right)$,

onde

$$
\Psi_{6}\left(\underset{\sim}{\alpha}, \beta_{1}, \ldots, \beta_{J}\right)=\exp \left\{\beta_{k l} a_{k l}-\sum_{i=1}^{n} \log \left[1+\exp \left(\alpha_{i}+x_{i j} \beta_{j}\right)\right]\right\},
$$

$a_{k l}=\sum_{i=1}^{n} x_{i l k} y_{i l}$

$\underset{\sim}{\beta}$ definido em (2.12)

$\stackrel{\sim}{k l}=1, \ldots, p_{j} ; \quad l, j=1, \ldots, J$

(v)

$\pi\left(\mu_{k} \mid \underset{\sim}{\alpha}, \beta_{1}, \ldots, \beta_{J}, \mu_{\underline{k}}, \stackrel{\sigma}{\sim}^{2}, p_{1}, \underset{\sim}{z}, \mathcal{D}\right) \propto \exp \left\{-\frac{1}{2 e_{k}^{2}}\left(\mu_{k}-d_{k}\right)^{2}\right\} \cdot \prod_{i=1}^{n} \exp \left\{-\frac{z_{i k}}{2 \sigma_{k}^{2}}\left(\alpha_{i}-\mu_{k}\right)^{2}\right\}$

Isto é

$\pi\left(\mu_{k} \mid \underset{\sim}{\alpha}, \beta_{1}, \ldots, \beta_{J}, \mu_{\underline{k}},{\underset{\sim}{2}}^{2}, p_{1}, \mathcal{D}, \underset{\sim}{z}\right) \sim N\left\{\frac{d_{k} \sigma_{k}^{2}+e_{k}^{2} \sum_{i=1}^{n} \alpha_{i} Z_{i k}}{\sigma_{k}^{2}+e_{k}^{2} \sum_{i=1}^{n} Z_{i k}}, \frac{e_{k}^{2} \sigma_{k}^{2}}{\sigma_{k}^{2}+e_{k}^{2} \sum_{i=1}^{n} Z_{i k}}\right\}$,

onde $k \neq \underline{k}, \quad k, \underline{k}=1,2$.

Observaçōes:

- Considerando $\Theta=\left(\underset{\sim}{\alpha}, \beta_{1}, \ldots, \beta_{J} \underset{\sim}{\mu} \underset{\sim}{\sigma^{2}}, p_{1}\right)$, para gerar amostras da distribuição à posteriori conjunta (2.22) usamos o algoritmo Gibbs sampling. Começamos o método iterativo com valores iniciais $\Theta_{\sim}^{(0)}=\left(\Theta_{1}^{(0)}, \ldots, \Theta_{2}^{(0)}\right)$, e seguindo os seguintes passos: 
(i) Geramos uma amostra $\underset{\sim}{z^{(1)}}=\left({\underset{\sim}{\sim_{1}}}_{(1)}^{(1)}, \ldots, \underset{\sim_{2}}{z^{(1)}}\right)$ da equação $(2.21)$.

(ii) Geramos uma amostra de $\underset{\sim}{\Theta}$ das distribuições condicionais

$$
\begin{aligned}
& \pi\left(\Theta_{1} \mid \Theta_{2}^{(0)}, \ldots, \Theta_{p}^{(0)}, \underset{\sim}{z^{(1)}}, \underset{\sim}{x}, \underset{\sim}{z}\right), \\
& \pi\left(\Theta_{2} \mid \Theta_{1}^{(1)}, \Theta_{3}^{(0)}, \ldots, \Theta_{p}^{(0)}, \underset{\sim}{z^{(1)}}, \underset{\sim}{x}, \underset{\sim}{z}\right), \\
& \vdots \\
& \pi\left(\Theta_{p} \mid \Theta_{1}^{(1)}, \ldots, \Theta_{p-1}^{(1)},{\underset{\sim}{z}}^{(1)}, \underset{\sim}{x}, \underset{\sim}{z}\right),
\end{aligned}
$$

Então continuamos as iterações repetindo os passos (i) e (ii).

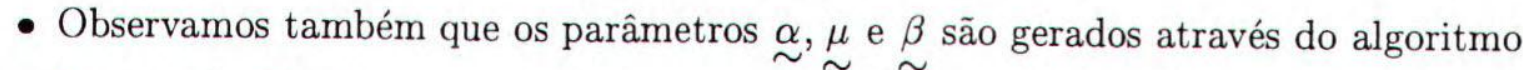
Metropolis-Hastings. 


\section{Capítulo 3}

\section{ANÁLISE DO MODELO PROBITO}

No estudo de modelos de regressão para o caso de onde a variável resposta $Y_{i j}$ é binária, com distribuição de $\operatorname{Bernoulli}\left(\theta_{i j}\right),(i=1,2, \ldots, n ; j=1,2, \ldots, J)$, a probabilidade de sucesso $\theta_{i j}$ pode variar em função de um vetor de covariáveis $x_{i j}$. Como uma alternativa ao modelo de regressão logística consideramos neste capítulo a função de ligação probito ou função Normal inversa.

Considerar $Y_{i j}(1$ ou 0$)$ a resposta para $i$-ésima observação no $j$-ésimo tempo, com uma distribuição de Bernoulli com parâmetro $\theta_{i j}$, ou seja,

$$
\begin{array}{r}
\operatorname{Pr}\left[Y_{i j}=y_{i j} \mid \alpha_{i}, \beta_{j}, x_{i j}\right]=\theta_{i j}^{y_{i j}}\left(1-\theta_{i j}\right)^{1-y_{i j}} \\
i=1, \ldots, n ; \quad j=1, \ldots, J .
\end{array}
$$

onde $\theta_{i j}$ é dado por

$$
\theta_{i j}=\Phi\left(\alpha_{i}+x_{i j} \beta_{j}\right) \quad \text { (Probito) }
$$

e $\Phi(\cdot)$ é a função de distribuição Normal acumulada, isto é,

$$
\Phi\left(\alpha_{i}+x_{i j} \beta_{j}\right)=\frac{1}{\sqrt{2 \pi}} \int_{-\infty}^{\alpha_{i}+x_{i j} \beta_{j}} \exp \left\{-\frac{u^{2}}{2}\right\} d u
$$

e $\alpha_{i}$ denota um efeito aleatório na i-ésima observação, especificando a correlação entre $Y_{i 1}, Y_{i 2}, \ldots, Y_{i J}$. Assumimos inicialmente $\alpha_{i} \stackrel{i i d}{\sim} N\left(0, \sigma_{\alpha}^{2}\right)$.

Desta forma temos,

$$
\begin{array}{r}
\operatorname{Pr}\left[Y_{i j}=y_{i j} \mid \alpha_{i}, \beta_{j}, x_{i j}\right]=\Phi\left(\alpha_{i}+x_{i j} \beta_{j}\right)^{y_{i j}}\left[1-\Phi\left(\alpha_{i}+x_{i j} \beta_{j}\right)\right]^{1-y_{i j}} \\
i=1, \ldots, n ; \quad j=1, \ldots, J .
\end{array}
$$


Assumimos também que as observações são independentes, pois a correlação entre os tempos para cada observação é capturado pelo efeito aleatório $\alpha_{i}$. Neste caso a função de verossimilhança é dada por,

$$
\mathrm{L}\left(\underset{\sim}{\alpha}, \beta_{1}, \beta_{2}, \ldots, \beta_{J} \mid \mathcal{D}\right)=\prod_{i=1}^{n} \prod_{j=1}^{J} \Phi\left(\alpha_{i}+x_{i j} \beta_{j}\right)^{y_{i j}}\left[1-\Phi\left(\alpha_{i}+x_{i j} \beta_{j}\right)\right]^{1-y_{i j}}
$$

onde $\underset{\sim}{\alpha}=\left(\alpha_{1}, \alpha_{2}, \ldots, \alpha_{n}\right)$ e $\mathcal{D}$ representa o conjunto de dados $\left(x_{i j}, y_{i j}\right), i=1, \ldots, n$; $j=1, \ldots, J$.

\subsection{Análise Clássica do Modelo}

Inicialmente, faremos as inferências sobre os parâmetros do modelo a partir do método de verossimilhança. Considerando $\alpha_{i}(i=1, \ldots, n)$ igual à $\alpha$, ou seja, um parâmetro de efeito fixo, estamos assumindo que nāo há correlação entre os tempos.

Como nosso objetivo é encontrar estimadores para os parâmetros que maximizem a função de verossimilhança utilizaremos o logarítmo da funçāo de verossimilhança. Para simplificação da notação escrevemos $\Phi$ ao invés de $\Phi\left(\alpha+x_{i j} \beta_{j}\right)$. Assim, temos:

$$
\begin{aligned}
\ell\left(\alpha, \beta_{1}, \beta_{2}, \ldots, \beta_{J} \mid \mathcal{D}\right) & =\log \mathrm{L}\left(\alpha, \beta_{1}, \beta_{2}, \ldots, \beta_{J} \mid \mathcal{D}\right) \\
& =\sum_{i=1}^{n} \sum_{j=1}^{J}\left\{y_{i j} \log \Phi+\left(1-y_{i j}\right) \log (1-\Phi)\right\}
\end{aligned}
$$

As derivadas de primeira ordem do $\log$ da função de verossimilhança $(\ell)$ são dadas por,

$$
\begin{aligned}
& \frac{\partial \ell}{\partial \alpha}=\sum_{i=1}^{n} \sum_{j=1}^{J}\left\{\frac{\Phi_{\alpha}^{\prime}\left(y_{i j}-\Phi\right)}{\Phi(1-\Phi)}\right\} \\
& \frac{\partial \ell}{\partial \beta_{p_{j} j}}=\sum_{i=1}^{n}\left\{\frac{\Phi_{\beta_{p_{j}}}^{\prime}\left(y_{i j}-\Phi\right)}{\Phi(1-\Phi)}\right\} \\
& j=1,2, \ldots, J
\end{aligned}
$$


Fazendo $\frac{\partial \ell}{\partial \alpha}=0$ e $\frac{\partial \ell}{\partial \beta_{p^{l}}}=0,(j=1,2, \ldots, J)$ temos as equações de Escore, cujas soluções são os possíveis estimadores de máxima verossimilhança e podem ser encontrados utilizando o método numérico de Newton-Raphson.

As derivadas de segunda ordem para matriz de informação de Fisher, necessária para para o método de Newton-Raphson, são dadas por,

$\frac{\partial^{2} \ell}{\partial \alpha^{2}}=\sum_{i=1}^{n} \sum_{j=1}^{J} \frac{\left[\Phi_{\alpha}^{\prime}\left(y_{i j}-\Phi\right)-\Phi_{\alpha}^{\prime 2}\right] \Phi(1-\Phi)-\phi_{\alpha}^{2}(1-2 \Phi)\left(y_{i j}-\Phi\right)}{[\Phi(1-\Phi)]^{2}}$

$\frac{\partial^{2} \ell}{\partial \beta_{p_{j} j}^{2}}=\sum_{i=1}^{n} \frac{\left[\Phi_{\beta_{p_{j} j}}^{\prime}\left(y_{i j}-\Phi\right)-\Phi_{\beta_{p_{j} j}}^{\prime 2}\right] \Phi(1-\Phi)-{\phi^{\prime}}_{\beta_{p_{j} j}}^{2}(1-2 \Phi)\left(y_{i j}-\Phi\right)}{[\Phi(1-\Phi)]^{2}}$

$j=1,2, \ldots, J$

$\frac{\partial^{2} \ell}{\partial \alpha \partial \beta_{p_{j} j}}=\sum_{i=1}^{n} \frac{\left[\Phi^{\prime \prime}{ }_{\alpha \beta_{p_{j} j}}\left(y_{i j}-\Phi\right)-\Phi_{\beta_{p_{j} j}}^{\prime} \Phi_{\alpha}^{\prime}\right] \Phi(1-\Phi)-\Phi_{\beta_{p_{j} j}}^{\prime} \Phi_{\alpha}^{\prime}(1-2 \Phi)\left(y_{i j}-\Phi\right)}{[\Phi(1-\Phi)]^{2}}$

$j=1,2, \ldots, J$

$\frac{\partial^{2} \ell}{\partial \beta_{p_{j} j} \partial \beta_{p_{k} j}}=0 \quad$ para $j \neq k$ onde $j=1,2, \ldots, J ; \quad k=1,2, \ldots, J$

$\frac{\partial^{2} \ell}{\partial \beta_{p_{j} j} \partial \beta_{p_{k} k}}=0 \quad$ para $j \neq k$ onde $j=1,2, \ldots, J ; \quad k=1,2, \ldots, J$

$\frac{\partial^{2} \ell}{\partial \beta_{p_{j} j} \partial \beta_{p_{k} k}}=\sum_{i=1}^{n} \frac{\left[\Phi^{\prime \prime}{ }_{\beta_{p_{j} j} \beta_{p_{k} k}}\left(y_{i j}-\Phi\right)-\Phi_{\beta_{p_{k} k}}^{\prime} \Phi_{\beta_{p_{j} j}}^{\prime}\right] \Phi(1-\Phi)-\Phi_{\beta_{p_{k} k}}^{\prime} \Phi_{\beta_{p_{j} j}}^{\prime}(1-2 \Phi)\left(y_{i j}-\Phi\right)}{[\Phi(1-\Phi)]^{2}}$,

$j=k \quad$ onde $\quad j=1,2, \ldots, J ; \quad k=1,2, \ldots, J$.

Definir, 


$$
\begin{aligned}
& \Phi_{\alpha}^{\prime}=\frac{\partial \Phi\left(\alpha+x_{i j} \beta_{j}\right)}{\partial \alpha}=\frac{1}{\sqrt{2 \pi}} \exp \left\{-\frac{\left(\alpha+x_{i j} \beta_{j}\right)^{2}}{2}\right\} \\
& i=1, \ldots, n ; j=1,2, \ldots, J \\
& \Phi^{\prime \prime}{ }_{\alpha}=\frac{\partial^{2} \Phi\left(\alpha+x_{i j} \beta_{j}\right)}{\partial \alpha^{2}}=\frac{1}{\sqrt{2 \pi}} \exp \left\{-\frac{\left(\alpha+x_{i j} \beta_{j}\right)^{2}}{2}\right\}\left[-\left(\alpha+x_{i j} \beta_{j}\right)\right] \\
& i=1, \ldots, n ; \quad j=1,2, \ldots, J \\
& \Phi_{\beta_{p_{j} j}}^{\prime}=\frac{\partial \Phi\left(\alpha+x_{i j} \beta_{j}\right)}{\partial \beta_{p_{j} j}}=\frac{1}{\sqrt{2 \pi}} \exp \left\{-\frac{\left(\alpha+x_{i j} \beta_{j}\right)^{2}}{2}\right\} x_{i p_{j}} \\
& i=1, \ldots, n ; \quad j=1,2, \ldots, J \\
& \Phi_{\beta_{p_{j} j}}^{\prime \prime}=\frac{\partial^{2} \Phi\left(\alpha+x_{i j} \beta_{j}\right)}{\partial \beta_{p_{j} j}^{2}}=\frac{1}{\sqrt{2 \pi}} \exp \left\{-\frac{\left(\alpha+x_{i j} \beta_{j}\right)^{2}}{2}\right\}\left[-\left(\alpha+x_{i j} \beta_{j}\right)\right] x_{i j p_{j}}^{2} \\
& i=1, \ldots, n ; \quad j=1,2, \ldots, J \\
& \Phi_{\alpha \beta_{p_{j} j}}^{\prime \prime}=\frac{\partial^{2} \Phi\left(\alpha+x_{i j} \beta_{j}\right)}{\partial \alpha \beta_{p_{j} j}}=\frac{1}{\sqrt{2 \pi}} \exp \left\{-\frac{\left(\alpha+x_{i j} \beta_{j}\right)^{2}}{2}\right\}\left[-\left(\alpha+x_{i j} \beta_{j}\right)\right] x_{i j p_{j}} \\
& i=1, \ldots, n ; j=1,2, \ldots, J \\
& \Phi^{\prime \prime}{ }_{\beta_{p_{j}} \beta_{p_{k} k}}=\frac{\partial^{2} \Phi\left(\alpha+x_{i j} \beta_{j}\right)}{\partial \beta_{p_{j} j} \beta_{p_{k} k}}=\frac{1}{\sqrt{2 \pi}} \exp \left\{-\frac{\left(\alpha+x_{i j} \beta_{j}\right)^{2}}{2}\right\}\left[-\left(\alpha+x_{i j} \beta_{j}\right)\right] x_{i j p_{j}} x_{i j p_{k}} \\
& i=1, \ldots, n ; \quad j=k ; \quad j, k=1,2, \ldots, J
\end{aligned}
$$

\subsection{Análise Bayesiana do Modelo}

Modelo 4: Modelo probito com efeito fixo.

Assumir os efeitos fixos, $\alpha_{i}(i=1, \ldots, n)$ iguais à $\alpha$, com as seguintes distribuições 
à priori:

(i) $\alpha \sim N\left(\mu_{0}, \sigma_{0}^{2}\right) \quad$ com $\mu_{0}$ e $\sigma_{0}^{2}$ conhecidos;

(ii) $\beta_{k j} \sim N\left(b_{k j}, c_{k j}^{2}\right)$ com $b_{k j}$ e $c_{k j}^{2}$ conhecidos;

$k=1,2, \ldots, p_{j} ; \quad j=1,2, \ldots, J$.

Assumindo indepêndencia das distribuições a priori, temos a distribuição à priori conjunta dada por,

$$
\pi\left(\alpha, \beta_{1}, \beta_{2}, \ldots, \beta_{J}\right) \propto \exp \left\{-\frac{\left(\alpha-\mu_{0}\right)^{2}}{2 \sigma_{0}^{2}}\right\} \prod_{j=1}^{J} \prod_{k=1}^{p_{j}} \exp \left\{-\frac{\left(\beta_{k j}-\mu_{k j}\right)^{2}}{2 \sigma_{k j}^{2}}\right\}
$$

Combinando (3.2) com (3.5), temos a seguinte distribuição à posteriori conjunta:

$$
\begin{aligned}
\pi\left(\alpha, \beta_{1}, \beta_{2}, \ldots, \beta_{J} \mid \mathcal{D}\right) \propto \exp \left[-\frac{\left(\alpha-\mu_{0}\right)^{2}}{2 \sigma_{0}^{2}}\right]\left\{\prod_{j=1}^{J} \prod_{k=1}^{p_{j}} \exp \left[-\frac{\left(\beta_{k j}-\mu_{k j}\right)^{2}}{2 \sigma_{k j}^{2}}\right]\right\} \\
\cdot\left\{\prod_{i=1}^{n} \prod_{j=1}^{J} \Phi\left(\alpha+x_{i j} \beta_{j}\right)^{y_{i j}}\left[1-\Phi\left(\alpha+x_{i j} \beta_{j}\right)\right]^{1-y_{i j}}\right\}
\end{aligned}
$$

As distribuições condicionais para o algoritmo de Metropolis-Hastings são dadas por:

(i)

$$
\begin{aligned}
& \pi\left(\alpha \mid \beta_{1}, \beta_{2}, \ldots, \beta_{J}, \mathcal{D}\right) \propto \exp \left[-\frac{\left(\alpha-\mu_{0}\right)^{2}}{2 \sigma_{0}^{2}}\right] \prod_{i=1}^{n} \prod_{j=1}^{J} \Phi\left(\alpha+x_{i j} \beta_{j}\right)^{y_{i j}\left[1-\Phi\left(\alpha+x_{i j} \beta_{j}\right)\right]^{1-y_{i j}}} \\
& \pi\left(\alpha \mid \beta_{1}, \beta_{2}, \ldots, \beta_{J}, \mathcal{D}\right) \propto N\left(\mu_{0}, \sigma_{0}^{2}\right) \mathrm{L}\left(\alpha, \beta_{1}, \beta_{2}, \ldots, \beta_{J} \mid \mathcal{D}\right)
\end{aligned}
$$


(ii)

$$
\begin{aligned}
& \pi\left(\beta_{k l} \mid \alpha, \beta_{1}, \ldots, \beta_{j-1}, \underset{\sim_{k l}}{\beta}, \beta_{j+1}, \ldots, \beta_{J}, \mathcal{D}\right) \propto \exp \left[-\frac{\left(\beta_{k l}-\mu_{k l}\right)^{2}}{2 \sigma_{k l}^{2}}\right] \\
& \cdot\left\{\prod_{i=1}^{n} \Phi\left(\alpha+x_{i j} \beta_{j}\right)^{y_{i j}}\left[1-\Phi\left(\alpha+x_{i j} \beta_{j}\right)\right]^{1-y_{i j}}\right\}
\end{aligned}
$$$$
\pi\left(\beta_{k l} \mid \alpha, \beta_{1}, \ldots, \beta_{j-1}, \underset{\sim}{\beta}, \beta_{j l+1}, \ldots, \beta_{J}, \mathcal{D}\right) \propto N\left(b_{k l}, c_{k l}^{2}\right) \cdot \Psi_{1}\left(\alpha, \beta_{1}, \beta_{2}, \ldots, \beta_{J}\right)
$$

onde

$$
\begin{aligned}
& \Psi_{1}\left(\alpha, \beta_{1}, \beta_{2}, \ldots, \beta_{J}\right)=\left\{\prod_{i=1}^{n} \Phi\left(\alpha+x_{i j} \beta_{j}\right)^{y_{i j}}\left[1-\Phi\left(\alpha+x_{i j} \beta_{j}\right)\right]^{1-y_{i j}}\right\} \\
& k=1, \ldots, p_{j} ; \quad l, j=1, \ldots, J ; \quad \text { e }{\underset{\sim}{k l}}_{k l} \text { definido em }(2.12)
\end{aligned}
$$

\subsection{Análise Bayesiana Assumindo uma Distribuição Normal para os efeitos aleatórios $\alpha_{i}$}

Modelo 5: Modelo probito assumindo efeitos aleatórios normais $-\alpha_{i} \stackrel{i i d}{\sim} N\left(0, \sigma_{\alpha}^{2}\right)$;

$$
i=1, \ldots, n
$$

Como no Capítulo 2, assumir que $\alpha_{i}, \quad i=1, \ldots, n$ sejam efeitos aleatórios independentes com distribuição normal, $N\left(0, \sigma_{\alpha}^{2}\right)$ com as seguintes distribuições a priori,

(i) $\sigma_{\alpha}^{2} \sim \mathcal{I G}(a, b) \quad$ com $a$ e $b$ conhecidos;

(ii) $\beta_{l j} \sim N\left(b_{k j}, c_{k j}^{2}\right) \quad$ com $b_{l j}$ e $c_{l j}^{2}$ conhecidos; $l=1, \ldots, p_{j} ; j=1, \ldots, J$,

onde $\mathcal{I G}(a, b)$ denota a distribuição gama invertida com média $\frac{1}{b(a-1)}$ para $a>1$ e variância $\frac{1}{b^{2}(a-1)^{2}(a-2)}$ para $b>1$.

Assumindo independência das distribuições a priori e considerando a função de verossimilhança (3.2), a distribuição a posteriori conjunta para $\underset{\sim}{\alpha}=\left(\alpha_{1}, \ldots, \alpha_{n}\right), \quad \sigma_{\alpha}^{2} \mathrm{e}$ 
$\beta_{1}, \ldots, \beta_{J}$ é dada por,

$$
\begin{aligned}
\pi\left(\underset{\sim}{\alpha}, \sigma_{a}^{2}, \beta_{1}, \ldots, \beta_{J} \mid \mathcal{D}\right) \propto & \\
\propto & \left(\sigma_{\alpha}^{2}\right)^{-\left(\frac{n}{2}+a+1\right)} \exp \left\{-\frac{1}{2 \sigma_{\alpha}^{2}}\left[\frac{2}{b}+\sum_{i=1}^{n} \alpha_{i}^{2}\right]\right\} \\
& \cdot\left[\prod_{k=1}^{p_{j}} \prod_{j=1}^{J} \exp \left\{-\frac{1}{2 c_{k j}^{2}}\left(\beta_{k j}-b_{k j}\right)^{2}\right\}\right] \\
& \left.\cdot\left[\prod_{i=1}^{n} \prod_{j=1}^{J} \Phi\left(\alpha_{i}+x_{i j} \beta_{j}\right)^{y_{i j}}\left[1-\Phi\left(\alpha_{i}+x_{i j} \beta_{j}\right)\right]^{1-y_{i j}}\right\}\right] .
\end{aligned}
$$

Portanto as distribuiçōes condicionais para o algoritmo Metropolis-Hastings, são dadas por,

i)

$$
\pi\left(\alpha_{i} \mid \sigma_{\alpha}^{2}, \beta_{1}, \ldots, \beta_{J}, \mathcal{D}\right) \propto \exp \left\{-\frac{\alpha_{i}^{2}}{2 \sigma_{\alpha}^{2}}\right\} \prod_{j=1}^{J} \Phi\left(\alpha_{i}+x_{i j} \beta_{j}\right)^{y_{i j}}\left[1-\Phi\left(\alpha_{i}+x_{i j} \beta_{j}\right)\right]^{1-y_{i j}}
$$

para $i=1, \ldots, n$, isto é,

$$
\pi\left(\alpha_{i} \mid \sigma_{\alpha}^{2}, \beta_{1}, \ldots, \beta_{J}, \mathcal{D}\right) \propto N\left\{0, \sigma_{\alpha}^{2}\right\} \Psi_{2}\left(\alpha_{i}, \beta_{1}, \ldots, \beta_{J}\right)
$$

onde

$$
\Psi_{2}\left(\alpha_{i}, \beta_{1}, \ldots, \beta_{J}\right)=\prod_{j=1}^{J} \Phi\left(\alpha_{i}+x_{i j} \beta_{j}\right)^{y_{i j}}\left[1-\Phi\left(\alpha_{i}+x_{i j} \beta_{j}\right)\right]^{1-y_{i j}}
$$

ii)

$$
\begin{aligned}
\pi\left(\sigma_{\alpha}^{2} \mid \alpha_{i}, \beta_{1}, \ldots, \beta_{J}, \mathcal{D}\right) & \propto\left(\sigma_{\alpha}^{2}\right)^{-\left(\frac{n}{2}+a+1\right)} \exp \left\{-\frac{1}{\sigma_{\alpha}^{2}}\left[\frac{1}{b}+\frac{1}{2} \sum_{i=1}^{n} \alpha_{i}^{2}\right]\right\} \\
& \propto\left(\sigma_{\alpha}^{2}\right)^{-\left(\frac{n}{2}+a+1\right)} \exp \left\{-\frac{1}{\sigma_{\alpha}^{2}\left[\frac{1}{b}+\frac{1}{2} \sum_{i=1}^{n} \alpha_{i}^{2}\right]^{-1}}\right\}
\end{aligned}
$$

isto é,

$$
\sigma_{\alpha}^{2} \mid \alpha_{i}, \beta_{1}, \ldots, \beta_{J}, \mathcal{D} \sim \mathcal{I G}\left[\frac{n}{2}+a ;\left(\frac{1}{b}+\frac{1}{2} \sum_{i=1}^{n} \alpha_{i}^{2}\right)^{-1}\right]
$$


iii)

$\pi\left(\beta_{k l} \mid \alpha_{i}, \beta_{1}, \ldots, \beta_{j-1}, \underset{\sim}{\beta}, \beta_{j l+1}, \ldots, \beta_{J}, \mathcal{D}\right) \propto N\left(b_{k l}, c_{k l}^{2}\right) \cdot \Psi_{3}\left(\alpha_{i}, \beta_{1}, \beta_{2}, \ldots, \beta_{J}\right)$

onde

$$
\begin{aligned}
& \Psi_{3}\left(\alpha_{i}, \beta_{1}, \beta_{2}, \ldots, \beta_{J}\right)=\prod_{i=1}^{n} \Phi\left(\alpha_{i}+x_{i j} \beta_{j}\right)^{y_{i j}}\left[1-\Phi\left(\alpha_{i}+x_{i j} \beta_{j}\right)\right]^{1-y_{i j}} \\
& k=1, \ldots, p_{j} ; \quad l, j=1, \ldots, J ; \quad \text { e }{\underset{\sim}{k l}}_{k l}^{\beta} \text { definido em }(2.12) .
\end{aligned}
$$

Observamos que a amostra para $\sigma_{\alpha}^{2}$ é gerada diretamente pelo algoritmo Gibbs sampling.

\subsection{Análise Bayesiana Assumindo uma Mistura de Normais para $\alpha_{i}$}

Modelo 6: Modelo probito assumindo os efeitos aleatórios com uma mistura de distribuições Normais.

Consideramos nesta seção, uma mistura de duas distribuiçōes normais, para os efeitos aleatórios de $\alpha_{i}(i=1, \ldots, n)$, isto é,

$$
\pi\left(\alpha_{i}\right)=\sum_{k=1}^{2} p_{k} \Phi_{k}\left(\alpha_{i} \mid \mu_{k}, \sigma_{k}^{2}\right)
$$

onde $\sum_{k=1}^{2} p_{k}=1$ e $\Phi_{k}$ denota a densidade normal $N\left(\mu_{k}, \sigma_{k}^{2}\right)$.

Assumir independência à priori entre os parâmetros e considerar as seguintes densidades à priori para $\mu_{k}, \sigma_{k}^{2}, p_{1}$ e $\beta_{l j}, k=1,2 ; l=1, \ldots, p_{j} ; j=1, \ldots, J$ :

(i) $\mu_{k} \sim N\left(d_{k}, e_{k}^{2}\right) \quad$ com $d_{k}$ e $e_{k}$ conhecidos, $k=1,2$;

(ii) $\sigma_{k}^{2} \sim \mathcal{I G}\left(a_{k}, b_{k}\right) \quad$ com $a_{k}$ e $b_{k}$; conhecidos, $k=1,2$;

(iii) $\beta_{l j} \sim N\left(b_{l j}, c_{l j}^{2}\right) \quad \operatorname{com} b_{l j}$ e $c_{l j}^{2}$ conhecidos, $l=1, \ldots, p_{j} ; j=1, \ldots, J$

(iv) $p_{1} \sim \mathcal{B}(f, g)$ com $f$ e $g$ conhecidos 
onde $N\left(d_{k}, e_{k}\right)$ denota a distribuição normal com média $d_{k}$ e variância $e_{k} ; \mathcal{I G}\left(a_{k}, b_{k}\right)$ uma distribuição gama invertida com média $\frac{1}{b_{k}\left(a_{k}-1\right)}$ para $a_{k}>1$ e variância $\frac{1}{\bar{b}_{k}^{2}\left[\left(a_{k}-1\right)^{2}\left(a_{k}-2\right)\right]}$ para $a_{k}>2$ e $B(f, g)$ denota uma distribuição beta com média $\frac{f}{(f+g)}$ e variância $\frac{f g}{(f+g)^{2}(f+g+1)}$.

A densidade à posteriori conjunta para $\Theta=\left(\underset{\sim}{\alpha}, p_{1}, \mu_{1}, \mu_{2}, \sigma_{1}^{2}, \sigma_{2}^{2}, \beta_{1}, \ldots, \beta_{J}\right)$ é dada por,

$$
\pi(\Theta \mid \mathcal{D}) \propto \Psi(\Theta)\left\{\prod_{i=1}^{n} \sum_{k=1}^{2} p_{k} \Phi_{k}\left(\alpha_{i} \mid \mu_{k}, \sigma_{k}^{2}\right)\right\}
$$

onde

$$
\begin{aligned}
\Psi(\Theta)= & \left\{\prod_{k=1}^{2}\left(\sigma_{k}^{2}\right)^{-\left(a_{k}+1\right)} \exp \left(-\frac{b_{k}}{\sigma_{k}^{2}}\right)\right\} \cdot\left\{\prod_{k=1}^{2} \exp \left[-\frac{1}{2 e_{k}^{2}}\left(\mu_{k}-d_{k}\right)^{2}\right]\right\} . \\
& p_{I}^{f-1}\left(1-p_{1}\right)^{g-1} \cdot\left\{\prod_{l=1}^{p_{j}} \prod_{j=1}^{J} \exp \left[-\frac{1}{2 c_{l j}^{2}}\left(\beta_{l j}-b_{l j}\right)^{2}\right]\right\} \\
& \prod_{i=1}^{n} \prod_{j=1}^{J} \Phi\left(\alpha_{i}+x_{i j} \beta_{j}\right)^{y_{i j}}\left[1-\Phi\left(\alpha_{i}+x_{i j} \beta_{j}\right)\right]^{1-y_{i j}} .
\end{aligned}
$$

Considerando a introdução da variável artificial $\underset{\sim}{z}=\left(z_{i 1}, z_{i 2}\right), i=1, \ldots, n, \underset{\sim}{z}=$ $\left({\underset{\sim}{1}}_{1}, \ldots, \underset{\sim_{n}}{z}\right)$ (dada em $(2.21)$ ), a densidade à posteriori conjunta para $\Theta$ é dada por:

$$
\begin{aligned}
\pi(\Theta \mid \mathcal{D}, \underset{\sim}{z}) \propto \prod_{i=1}^{n} \prod_{k=1}^{2}\left[p_{k} \Phi_{k}\left(\alpha_{i} \mid \mu_{k}, \sigma_{k}^{2}\right)\right]^{z_{i k}} \cdot \\
\cdot\left\{\prod_{k=1}^{2}\left(\sigma_{k}^{2}\right)^{-\left(a_{k}+1\right)} \exp \left[\frac{-1}{b_{k} \sigma_{k}^{2}}\right]\right\} \cdot p_{1}^{f-1}\left(1-p_{1}\right)^{g-1} \\
\cdot\left\{\prod_{k=1}^{2} \exp \left[-\frac{1}{2 e_{k}^{2}}\left(\mu_{k}-d_{k}\right)^{2}\right]\right\} \cdot\left\{\prod_{l=1}^{p_{j}} \prod_{j=1}^{J} \exp \left[-\frac{1}{2 c_{l j}^{2}}\left(\beta_{l j}-b_{l j}\right)^{2}\right]\right\} \\
\cdot \prod_{i=1}^{n} \prod_{j=1}^{J} \Phi\left(\alpha_{i}+x_{i j} \beta_{j}\right)^{y_{i j}}\left[1-\Phi\left(\alpha_{i}+x_{i j} \beta_{j}\right)\right]^{1-y_{i j}}
\end{aligned}
$$

As distribuições condicionais para gerar amostras dos parâmetros através do algoritmo Metropolis-Hastings são dadas por: 
(i)

$\pi\left(\alpha_{i} \mid \beta_{1}, \ldots, \beta_{J} \underset{\sim}{\mu} \underset{\sim}{\sigma^{2}}, p_{1}, \underset{\sim}{z}, \mathcal{D}\right) \propto N\left\{\frac{Z_{i 1} \mu_{1} \sigma_{2}^{2}+Z_{i 2} \mu_{2} \sigma_{1}^{2}}{Z_{i 1} \sigma_{2}^{2}+Z_{i 2} \sigma_{1}^{2}}, \frac{\sigma_{1}^{2} \sigma_{2}^{2}}{Z_{i 1} \sigma_{2}^{2}+Z_{i 2} \sigma_{1}^{2}}\right\} \Psi_{4}\left(\alpha_{i}, \beta_{1}, \ldots, \beta_{J}\right)$, onde

$$
\begin{aligned}
& \Psi_{4}\left(\alpha_{i}, \beta_{1}, \ldots, \beta_{J}\right)=\prod_{j=1}^{J} \Phi\left(\alpha_{i}+x_{i j} \beta_{j}\right)^{y_{i j}}\left[1-\Phi\left(\alpha_{i}+x_{i j} \beta_{j}\right)\right]^{1-y_{i j}}, \\
& \underset{\sim}{\mu}=\left(\mu_{1}, \mu_{2}\right) ; \quad \underset{\sim}{\sigma}=\left(\sigma_{1}^{2}, \sigma_{2}^{2}\right) ; \quad i=1,2, \ldots, n ;
\end{aligned}
$$

(ii)

$$
\begin{array}{r}
\pi\left(\sigma_{k}^{2} \mid \alpha_{i}, \beta_{1}, \ldots, \beta_{J} \underset{\sim}{\mu}, \sigma_{\underline{k}}^{2}, p_{1}, \underset{\sim}{z}, \mathcal{D}\right) \propto\left(\sigma_{k}^{2}\right)^{-\left(a_{k}+1\right)} \exp \left\{-\frac{1}{b_{k} \sigma_{k}^{2}}\right\}\left(\sigma_{k}^{2}\right)^{-\frac{v_{k}}{2}} \\
\cdot \exp \left\{-\frac{1}{2 \sigma_{k}^{2}} \sum_{i=1}^{n} z_{i k}\left(\alpha_{i}-\mu_{k}\right)^{2}\right\},
\end{array}
$$

onde $v_{k}=\sum_{i=1}^{n} z_{i k}$

Isto é,

$$
\sigma_{k}^{2} \mid \alpha_{i}, \beta_{1}, \ldots, \beta_{J}, \underset{\sim}{\mu}, p_{1}, \underset{\sim}{z}, \mathcal{D} \sim \mathcal{I} \mathcal{G}\left[a_{k}+\frac{v_{k}}{2} ;\left(\frac{1}{b_{k}}+\frac{\sum_{i=1}^{n} z_{i k}\left(\alpha_{i}-\mu_{k}\right)^{2}}{2}\right)^{-1}\right]
$$

onde $k \neq \underline{k}, \quad k, \underline{k}=1,2$

(iii)

$$
p_{1} \mid \underset{\sim}{\alpha}, \beta_{1}, \ldots, \beta_{J} \underset{\sim}{\mu} .^{2}, \underset{\sim}{\sim}, \mathcal{D} \sim \mathcal{B}\left(f+v_{1}, g+v_{2}\right)
$$

(iv)

$\pi\left(\beta_{k l} \mid \underset{\sim}{\alpha}, \beta_{1}, \ldots, \beta_{j-1}, \underset{\sim}{\beta}, \beta_{j+1}, \ldots, \beta_{J}, \underset{\sim}{\mu} \underset{\sim}{\sigma^{2}}, p_{1}, \underset{\sim}{z}, \mathcal{D}\right) \propto N\left(b_{k l}, c_{k l}^{2}\right) \Psi_{5}\left(\underset{\sim}{\alpha}, \beta_{1}, \ldots, \beta_{J}\right)$,

onde

$$
\Psi_{5}\left(\underset{\sim}{\alpha}, \beta_{1}, \ldots, \beta_{J}\right)=\prod_{i=1}^{n} \Phi\left(\alpha_{i}+x_{i j} \beta_{j}\right)^{y_{i j}}\left[1-\Phi\left(\alpha_{i}+x_{i j} \beta_{j}\right)\right]^{1-y_{i j}},
$$

$\underset{\sim}{\beta}$ definido em (2.12)

$\tilde{k}_{k l}=1, \ldots, p_{j} ; \quad l, j=1, \ldots, J$ 
(v)

$$
\pi\left(\mu_{k} \mid \underset{\sim}{\alpha}, \beta_{1}, \ldots, \beta_{J}, \mu_{\underline{k}}, \underset{\sim}{\sigma^{2}}, p_{1}, \mathcal{D}, \underset{\sim}{z}\right) \sim N\left\{\frac{d_{k} \sigma_{k}^{2}+e_{k}^{2} \sum_{i=1}^{n} \alpha_{i} Z_{i k}}{\sigma_{k}^{2}+e_{k}^{2} \sum_{i=1}^{n} Z_{i k}}, \frac{e_{k}^{2} \sigma_{k}^{2}}{\sigma_{k}^{2}+e_{k}^{2} \sum_{i=1}^{n} Z_{i k}}\right\},
$$

onde $k \neq \underline{k}, \quad k, \underline{k}=1,2$

Para gerar as amostras usamos o mesmo procedimento descrito no cap. 2 seção 5. 
38 


\section{Capítulo 4}

\section{DADOS BINÁRIOS \\ CORRELACIONADOS COM RÉPLICAS}

Muitas vezes em um banco de dados de muitas unidades experimentais podemos verificar que alguns resultados repetidos ocorrem com uma dada frequência. Neste capítulo trataremos desse caso utilizando a mesma modelagem anterior inclúndo a frequência dessas ocorrências nos modelos.

Consideramos neste capítulo, um dado número de observações $N$, dentre as quais podemos ter repetições dos dados analisados (réplicas). As $N$ observações podem ser escritas como $n$ grupos considerando suas respectivas frequências, i.e., $N=\sum_{i=1}^{n} f_{i}$.

Assim $Y_{i j}(1$ ou 0$)$, a resposta para a $i$-ésima observação no $j$-ésimo tempo, segue distribuição de Bernoulli com parâmetro $\theta_{i j}$, ou seja,

$$
\begin{array}{r}
\operatorname{Pr}\left[Y_{i j}=y_{i j} \mid \alpha_{i}, \beta_{j}, x_{i j}\right]=\theta_{i j}^{y_{i j}}\left(1-\theta_{i j}\right)^{1-y_{i j}} \\
i=1, \ldots, N ; \quad j=1, \ldots, J .
\end{array}
$$

Assim temos duas possibilidades para modelar $\theta_{i j}$ :
a) $\theta_{i j}=\frac{\exp \left(\alpha_{i}+x_{i j} \beta_{j}\right)}{1+\exp \left(\alpha_{i}+x_{i j} \beta_{j}\right)}$ (Logístico)
b) $\theta_{i j}=\Phi\left(\alpha_{i}+x_{i j} \beta_{j}\right)$ (Probito)

Assumindo que as observaçōes são independentes, a função de verossimilhança é 
dada por,

$$
\mathrm{L}\left(\underset{\sim}{\alpha}, \beta_{1}, \beta_{2}, \ldots, \beta_{J} \mid \mathcal{D}\right)=\prod_{i=1}^{N} \prod_{j=1}^{J} \theta_{i j}^{y_{i j}}\left(1-\theta_{i j}\right)^{1-y_{i j}}
$$

onde $\underset{\sim}{\alpha}=\left(\alpha_{1}, \alpha_{2}, \ldots, \alpha_{N}\right)$ e $\mathcal{D}$ representa os dados $\left(y_{i j}, x_{i j}\right)$.

Como podemos escrever as $N$ observações em $n$ grupos de frequências $f_{i}, i=$ $1, \ldots, n$, a função de verossimilhança pode ser escrita na forma,

$$
E\left(\underset{\sim}{\alpha}, \beta_{1}, \beta_{2}, \ldots, \beta_{J} \mid \mathcal{D}\right)=\prod_{i=1}^{n} \prod_{j=1}^{J}\left[\theta_{i j}^{y_{i j}}\left(1-\theta_{i j}\right)^{1-y_{i j}}\right]^{f_{i}}
$$

onde $\underset{\sim}{\alpha}=\left(\alpha_{1}, \alpha_{2}, \ldots, \alpha_{n}\right)$

\subsection{Estimadores de Máxima Verossimilhança}

Considerando $\alpha_{i}(i=1,2, \ldots, n)$ iguais a $\alpha$, ou seja, efeitos fixos, e o modelo logístico, o logaritmo da função de verossimilhança é dada por,

$$
\begin{aligned}
\ell\left(\alpha, \beta_{1}, \beta_{2}, \ldots, \beta_{J} \mid \mathcal{D}\right) & =\log \mathrm{E}\left(\alpha, \beta_{1}, \beta_{2}, \ldots, \beta_{J} \mid \mathcal{D}\right) \\
& =\sum_{i=1}^{n} \sum_{j=1}^{J} \alpha y_{i j} f_{i}+\sum_{i=1}^{n} \sum_{j=1}^{J} x_{i j} \beta_{j} y_{i j} f_{i}-\sum_{i=1}^{n} \sum_{j=1}^{J} f_{i} \log \left[1+\exp \left(\alpha+x_{i j} \beta_{j}\right)\right] .
\end{aligned}
$$

Derivando o logaritmo da função verossimilhança em relação à $\alpha$ e $\beta$ e igualando à 0 (zero), temos as seguintes equações scores:

$$
\begin{aligned}
& \sum_{i=1}^{n} \sum_{j=1}^{J} y_{i j} f_{i}-\sum_{i=1}^{n} \sum_{j=1}^{J} f_{i} \frac{\exp \left\{\left(\widehat{\alpha}+x_{i j} \widehat{\beta}_{j}\right)\right\}}{1+\exp \left\{\widehat{\alpha}+x_{i j} \widehat{\beta}_{j}\right\}}=0 \\
& \sum_{i=1}^{n} x_{i j} y_{i j} f_{i}-\sum_{i=1}^{n} x_{i j p_{j}} f_{i} \frac{\exp \left\{\left(\widehat{\alpha}+x_{i j} \widehat{\beta}_{j}\right)\right\}}{1+\exp \left\{\widehat{\alpha}+x_{i j} \widehat{\beta}_{j}\right\}}=0 \\
& j=1,2, \ldots, J
\end{aligned}
$$


Para o caso do modelo probito $\left(\theta_{i j}=\Phi\left(\alpha+x_{i j} \beta_{j}\right)=\Phi\right)$ o logaritmo da função de verossimilhança é dado por,

$$
\begin{aligned}
\ell\left(\alpha, \beta_{1}, \beta_{2}, \ldots, \beta_{J} \mid \mathcal{D}\right) & =\log \mathrm{L}\left(\alpha, \beta_{1}, \beta_{2}, \ldots, \beta_{J} \mid \mathcal{D}\right) \\
& =\sum_{i=1}^{n} \sum_{j=1}^{J} f_{i}\left\{y_{i j} \log \Phi+\left(1-y_{i j}\right) \log (1-\Phi)\right\}
\end{aligned}
$$

As equações scores são dadas por,

$$
\begin{aligned}
& \sum_{i=1}^{n} \sum_{j=1}^{J} f_{i}\left\{\frac{\Phi_{\alpha}^{\prime}\left(y_{i j}-\Phi\right)}{\Phi(1-\Phi)}\right\}=0 \\
& \sum_{i=1}^{n} f_{i}\left\{\frac{\Phi_{\beta_{p_{j} j}}^{\prime}\left(y_{i j}-\Phi\right)}{\Phi(1-\Phi)}\right\}=0 \\
& j=1,2, \ldots, J
\end{aligned}
$$

$\operatorname{com} \Phi_{\alpha}^{\prime}$ e $\Phi_{\beta_{p_{j} j}}^{\prime}$ apresentadas em (3.3).

\subsection{Análise Bayesiana Assumindo $\alpha_{i}=\alpha$}

Para uma análise Bayesiana considerando $\alpha_{i}=\alpha$, assumir as seguintes densidades a priori informativas:

(i) $\alpha \sim N\left(\mu_{0}, \sigma_{0}^{2}\right)$ com $\mu_{0}$ e $\sigma_{0}^{2} \quad$ conhecidos;

(i) $\beta_{k j} \sim N\left(\mu_{k j}, \sigma_{k j}^{2}\right) \quad$ com $\mu_{k j}$ e $\sigma_{k j}^{2} \quad$ conhecidos;

$$
j=1,2, \ldots, J \quad k=1,2, \ldots, p_{j} .
$$

Com independência das distribuiçōes a priori obtemos a seguinte densidade a posteriori conjunta para os parâmetros:

$$
\begin{aligned}
\pi\left(\alpha, \beta_{1}, \beta_{2}, \ldots, \beta_{J} \mid \mathcal{D}\right) \propto & {\left[\exp \left\{-\frac{\left(\alpha-\mu_{0}\right)^{2}}{2 \sigma_{0}^{2}}\right\} \prod_{j=1}^{J} \prod_{k=1}^{p_{j}} \exp \left\{-\frac{\left(\beta_{k j}-\mu_{k j}\right)^{2}}{2 \sigma_{k j}^{2}}\right\}\right] } \\
& \cdot\left\{\prod_{i=1}^{n} \prod_{j=1}^{J}\left[\theta_{i j}^{y_{i j}}\left(1-\theta_{i j}\right)^{1-y_{i j}}\right]^{f_{i}}\right\}
\end{aligned}
$$

Com a ligação logística, as distribuiçōes condicionais para o algoritimo de Gibbs sampling são dadas por, 
(i)

$$
\pi\left(\alpha \mid \beta_{1}, \beta_{2}, \ldots, \beta_{J}, \mathcal{D}\right) \propto N\left(\mu_{0}, \sigma_{0}^{2}\right) \cdot \Psi_{1}\left(\alpha, \beta_{1}, \beta_{2}, \ldots, \beta_{J}\right)
$$

onde

$$
\Psi_{1}\left(\alpha, \beta_{1}, \beta_{2}, \ldots, \beta_{J}\right)=\exp \left\{\alpha \sum_{i=1}^{n} \sum_{j=1}^{J} f_{i} y_{i j}-\sum_{i=1}^{n} \sum_{j=1}^{J} f_{i} \log \left[1+\exp \left(\alpha+x_{i j} \beta_{j}\right)\right]\right\}
$$

(ii)

$$
\begin{aligned}
& \pi\left(\beta_{k l} \mid \alpha, \beta_{1}, \ldots, \beta_{j-1},{\underset{\sim}{\beta k l}}_{k j+1}^{\beta}, \beta_{j+1}, \ldots, \beta_{J}, \mathcal{D}\right) \propto \\
& \propto N\left(\mu_{k l}, \sigma_{k l}^{2}\right) \cdot \Psi_{2}\left(\alpha, \beta_{1}, \beta_{2} \ldots, \beta_{J}, \mathcal{D}\right)
\end{aligned}
$$

onde

$$
\Psi_{2}\left(\alpha, \beta_{1}, \beta_{2} \ldots, \beta_{J}, \mathcal{D}\right)=\exp \left\{a_{k l} \beta_{k l}-\sum_{i=1}^{n} f_{i} \log \left[1+\exp \left(\alpha+x_{i j} \beta_{j}\right)\right]\right\} ;
$$

$a_{k l}=\sum_{i=1}^{n} x_{i l k} y_{i l} f_{i} ; \quad k=1, \ldots, p_{j} ; \quad l, j=1, \ldots, J$

e $\sim_{\sim k l}^{\beta}$ definido em (2.12).

Com a ligação probito, temos,

$$
\Psi_{1}\left(\alpha, \beta_{1}, \beta_{2}, \ldots, \beta_{J}\right)=\prod_{i=1}^{n} \prod_{j=1}^{J}\left[\Phi\left(\alpha+x_{i j} \beta_{j}\right)^{y_{i j}}\left[1-\Phi\left(\alpha+x_{i j} \beta_{j}\right)\right]^{1-y_{i j}}\right]^{f_{i}},
$$

e

$$
\begin{aligned}
\Psi_{2}\left(\alpha, \beta_{1}, \beta_{2}, \ldots, \beta_{J}\right)= & \prod_{i=1}^{n}\left[\Phi\left(\alpha+x_{i j} \beta_{j}\right)^{y_{i j}}\left[1-\Phi\left(\alpha+x_{i j} \beta_{j}\right)\right]^{1-y_{i j}}\right]^{f_{i}}, \\
& j=1,2, \ldots, J .
\end{aligned}
$$

\subsection{Análise Bayesiana Assumindo $\alpha_{i} \sim N\left(0, \sigma_{\alpha}^{2}\right)$}

Para esta seção assumimos efeitos aleatórios para $\alpha_{i}(i=1, \ldots, n)$, com distribuição normal $N\left(0, \sigma_{\alpha}^{2}\right)$ e consideramos as seguintes distribuiçōes a priori,

(i) $\sigma_{\alpha}^{2} \sim \mathcal{I G}(a, b)$ com $a$ e $b$ conhecidos;

(ii) $\beta_{l j} \sim N\left(b_{l j}, c_{l j}^{2}\right) \quad \operatorname{com} b_{l j}$ e $c_{l j}^{2} \quad$ conhecidos;

$$
l=1, \ldots, p_{j} ; j=1, \ldots, J \text {. }
$$


Consideramos independencia das distribuiçōes a priori, e com (4.3) as distribuições condicionais para o algoritimo Gibbs sampling, considerando a ligação logística, são dadas por,

i)

$$
\pi\left(\alpha_{i} \mid \sigma_{\alpha}^{2}, \beta_{1}, \ldots, \beta_{J}, \mathcal{D}\right) \propto N\left\{0, \sigma_{\alpha}^{2}\right\} \Psi_{3}\left(\alpha_{i}, \beta_{1}, \ldots, \beta_{J}\right)
$$

onde

$$
\Psi_{3}\left(\alpha_{i}, \beta_{1}, \ldots, \beta_{J}\right)=\exp \left\{\alpha_{i} f_{i} \sum_{j=1}^{J} y_{i j}-\sum_{j=1}^{J} f_{i} \log \left[1+\exp \left(\alpha_{i}+x_{i j} \beta_{j}\right)\right]\right\}
$$

ii)

$$
\sigma_{\alpha}^{2} \mid \underset{\sim}{\alpha}, \beta_{1}, \ldots, \beta_{J}, \mathcal{D} \sim \mathcal{I} \mathcal{G}\left[\frac{n}{2}+a ;\left(\frac{1}{b}+\frac{1}{2} \sum_{i=1}^{n} \alpha_{i}^{2}\right)^{-1}\right]
$$

iii)

$$
\pi\left(\beta_{k l} \mid \underset{\sim}{\alpha}, \beta_{1}, \ldots, \beta_{j-1}, \underset{\sim}{\beta}, \beta_{j+1}, \ldots, \beta_{J}, \mathcal{D}\right) \propto N\left(b_{k l}, c_{k l}^{2}\right) \Psi_{4}\left(\underset{\sim}{\alpha}, \beta_{1}, \ldots, \beta_{J}\right)
$$

onde

$$
\Psi_{4}\left(\underset{\sim}{\alpha}, \beta_{1}, \beta_{2} \ldots, \beta_{J}, \mathcal{D}\right)=\exp \left\{a_{k l} \beta_{k l}-\sum_{i=1}^{n} f_{i} \log \left[1+\exp \left(\alpha+x_{i j} \beta_{j}\right)\right]\right\}
$$

$a_{k l}=\sum_{i=1}^{n} x_{i l k} y_{i l} f_{i} ; \quad k=1, \ldots, p_{j} ; \quad l, j=1, \ldots, J$

e $\underset{\sim}{\beta}$ definido em (2.12)

Considerando a ligação probito, as distribuições condicionais para o algoritimo Gibbs sampling serão as mesmas da ligação logistica com,

$$
\begin{aligned}
\Psi_{3}\left(\alpha_{i}, \beta_{1}, \beta_{2}, \ldots, \beta_{J}\right)= & \prod_{j=1}^{J}\left[\Phi\left(\alpha+x_{i j} \beta_{j}\right)^{y_{i j}}\left[1-\Phi\left(\alpha+x_{i j} \beta_{j}\right)\right]^{1-y_{i j}}\right]^{f_{i}} \\
& i=1,2, \ldots, n
\end{aligned}
$$

e

$$
\begin{aligned}
\Psi_{4}\left(\underset{\sim}{\alpha}, \beta_{1}, \beta_{2}, \ldots, \beta_{J}\right)= & \prod_{i=1}^{n}\left[\Phi\left(\alpha+x_{i j} \beta_{j}\right)^{y_{i j}}\left[1-\Phi\left(\alpha+x_{i j} \beta_{j}\right)\right]^{1-y_{i j}}\right]^{f_{i}} \\
& j=1,2, \ldots, J
\end{aligned}
$$




\subsection{Análise Bayesiana Assumindo uma Mistura de Normais para $\alpha_{i}$}

Novamente assumimos um modelo de regressão de efeitos aleatórios para dados binários correlacionados onde $\alpha_{i}(i=1,2, \ldots, n)$ é uma mistura de duas distribuições normais, dada por,

$$
\begin{aligned}
\pi\left(\alpha_{i}\right) & =\sum_{k=1}^{2} p_{k} \phi_{k}\left(\alpha_{i} \mid \mu_{k}, \sigma_{k}^{2}\right) \\
& =p_{1} \Phi_{1}\left(\alpha_{i} \mid \mu_{\mathrm{I}}, \sigma_{\mathrm{I}}^{2}\right)+\left(1-p_{\mathrm{I}}\right) \Phi_{2}\left(\alpha_{i} \mid \mu_{2}, \sigma_{2}^{2}\right)
\end{aligned}
$$

com a restrição $p_{1}+p_{2}=1 ; \phi_{k}$ denota uma densidade normal.

Consideramos as seguintes densidades a priori para os parâmetros, $\mu_{k}, \sigma_{k}^{2}, p_{1}, \beta_{l j}$, $k=1,2 ; l=1, \ldots, p_{j} ; j=1, \ldots, J:$

(i) $\quad \mu_{k} \sim N\left(d_{k}, e_{k}^{2}\right) \quad$ com $d_{k}$ e $e_{k}$ conhecidos, $k=1,2$;

(ii) $\sigma_{k}^{2} \sim \mathcal{I G}\left(a_{k}, b_{k}\right) \quad$ com $a_{k}$ e $b_{k}$; conhecidos, $k=1,2$;

(iii) $\beta_{l j} \sim N\left(b_{l j}, c_{l j}^{2}\right) \quad$ com $b_{l j}$ e $c_{l j}$; conhecidos,

$$
l=1, \ldots, p_{j} ; \quad j=1, \ldots, J
$$

(iv) $p_{1} \sim B(f, g)$ com $f$ e $g$; conhecidos;

A distribuição a posteriori conjunta para $\Theta=\left(\alpha_{1}, \ldots, a_{n}, \mu_{1}, \mu_{2}, p_{1}, \sigma_{1}^{2}, \sigma_{2}^{2}, \beta_{1}, \ldots, \beta_{J}\right)$ é dada por

$$
\pi(\Theta \mid \mathcal{D}) \propto \Psi(\Theta)\left\{\prod_{i=1}^{n} \sum_{k=1}^{2} p_{k} \phi_{k}\left(\alpha_{i} \mid \mu_{k}, \sigma_{k}^{2}\right)\right\}
$$

onde 


$$
\begin{aligned}
\Psi(\Theta)= & \left\{\prod_{k=1}^{2}\left(\sigma_{k}^{2}\right)^{-\left(a_{k}+1\right)} \exp \left(-\frac{1}{b_{k} \sigma_{k}^{2}}\right)\right\} \cdot\left\{\prod_{k=1}^{2} \exp \left[-\frac{1}{2 e_{k}^{2}}\left(\mu_{k}-d_{k}\right)^{2}\right]\right\} \\
& p_{1}^{f-1}\left(1-p_{1}\right)^{g-1} \cdot\left\{\prod_{l=1}^{p_{j}} \prod_{j=1}^{J} \exp \left[-\frac{1}{2 c_{l j}^{2}}\left(\beta_{l j}-b_{l j}\right)^{2}\right]\right\} \\
& \prod_{i=1}^{n} \prod_{j=1}^{J}\left[p_{i j}^{y_{i j}}\left(1-p_{i j}\right)^{1-y_{i j}}\right]^{f_{i}}
\end{aligned}
$$

Como anteriormente para simplificar as distribuições condicionais necessárias para o algoritmo de Gibbs sampling, introduzimos uma variável latente, $\underset{\sim}{z}=\left(z_{i 1}, z_{i 2}\right), \quad\left(z_{i 1}+\right.$ $\left.z_{i 2}=1\right), \quad i=1, \ldots, n$, com distribuição conjunta

$$
\pi\left({\underset{\sim}{1}}_{1}^{z}, \ldots, \underset{\sim_{n}}{z}\right) \propto \frac{\prod_{i=1}^{n} \prod_{k=1}^{2}\left[p_{k} \phi_{k}\left(\alpha_{i} \mid \mu_{k}, \sigma_{k}^{2}\right)\right]^{z_{i k}}}{\prod_{i=1}^{n}\left\{\sum_{k=1}^{2} p_{k} \Phi_{k}\left(\alpha_{i} \mid \mu_{k}, \sigma_{k}^{2}\right)\right\}}
$$

Combinando a equação (4.12) com (4.13), obtemos,

$$
\pi\left(\Theta,{\underset{\sim}{z}}_{1}^{z}, \ldots, \underset{\sim_{n}}{z} \mid \mathcal{D}\right) \propto \Psi(\Theta)\left\{\prod_{i=1}^{n} \prod_{k=1}^{2}\left[p_{k} \phi_{k}\left(\alpha_{i} \mid \mu_{k}, \sigma_{k}^{2}\right)\right]^{z_{i k}}\right\}
$$

Considerando a ligação logistica $\theta_{i j}=\frac{\exp \left(\alpha_{i}+x_{i j} \beta_{j}\right)}{1+\exp \left(\alpha_{i}+x_{i j} \beta_{j}\right)}$ as distribuições condicionais para o algoritmo Gibbs sampling, são dadas por,

(i)

$$
\begin{gathered}
\pi\left(\alpha_{i} \mid \beta_{1}, \ldots, \beta_{J}, \underset{\sim}{\mu}{\underset{\sigma}{\sim}}^{2}, p_{1}, \underset{\sim}{z}, \mathcal{D}\right) \propto N\left\{\frac{z_{i 1} \mu_{1} \sigma_{2}^{2}+z_{i 2} \mu_{2} \sigma_{1}^{2}}{z_{i 1} \sigma_{2}^{2}+z_{i 2} \sigma_{1}^{2}}, \frac{\sigma_{1}^{2} \sigma_{2}^{2}}{z_{i 1} \sigma_{2}^{2}+z_{i 2} \sigma_{1}^{2}}\right\} \\
\cdot \Psi_{5}\left(\alpha_{i}, \beta_{1}, \ldots, \beta_{J}\right),
\end{gathered}
$$

onde,

$$
\Psi_{5}\left(\alpha_{i}, \beta_{1}, \ldots, \beta_{J}\right)=\exp \left\{\alpha_{i} y_{i}, f_{i}-\sum_{j=1}^{J} f_{i} \log \left[1+\exp \left(\alpha_{i}+x_{i j} \beta_{j}\right)\right]\right\}
$$

para $i=1, \ldots, n$. 
(ii)

$\sigma_{k}^{2} \mid \alpha_{i}, \beta_{1}, \ldots, \beta_{J}, \underset{\sim}{\mu}, \sigma_{\underline{k}}^{2}, p_{1}, \underset{\sim}{z}, \mathcal{D} \sim \mathcal{I G}\left[a_{k}+\frac{v_{k}}{2} ;\left(\frac{1}{b_{k}}+\frac{\sum_{i=1}^{n} z_{i k}\left(\alpha_{i}-\mu_{k}\right)^{2}}{2}\right)^{-1}\right]$

onde $v_{k}=\sum_{i=1}^{n} z_{i k} ; \quad k \neq \underline{k}, \quad k, \underline{k}=1,2$

(iii)

$$
p_{1} \mid \underset{\sim}{\alpha}, \beta_{1}, \ldots, \beta_{J}, \underset{\sim}{\mu} \underset{\sim}{\sigma^{2}}, \underset{\sim}{z}, \mathcal{D} \sim \mathcal{B}\left(f+v_{1}, g+v_{2}\right)
$$

(iv)

$\pi\left(\beta_{k l} \mid \underset{\sim}{\alpha}, \beta_{1}, \ldots, \beta_{j-1}, \underset{\sim}{\beta}, \beta_{j+1}, \ldots, \beta_{J}, \underset{\sim}{\mu} \underset{\sim}{\sigma^{2}}, p_{1}, \underset{\sim}{z}, \mathcal{D}\right) \propto N\left(b_{k l}, c_{k l}^{2}\right) \Psi_{6}\left(\underset{\sim}{\alpha}, \beta_{1}, \ldots, \beta_{J}\right)$

onde

$$
\Psi_{6}\left(\underset{\sim}{\alpha}, \beta_{1}, \ldots, \beta_{J}\right)=\exp \left\{a_{k l} \beta_{k l}-\sum_{i=1}^{n} f_{i} \log \left[1+\exp \left(\alpha+x_{i j} \beta_{j}\right)\right]\right\}
$$

$a_{k l}=\sum_{i=1}^{n} x_{i l k} y_{i l} f_{i} ; \quad k=1, \ldots, p_{j} ; \quad l, j=1, \ldots, J$

e $\underset{\sim}{\beta}$ definido em (2.12)

(v)

$\pi\left(\mu_{k} \mid \underset{\sim}{\alpha}, \beta_{1}, \ldots, \beta_{J}, \mu_{\underline{k}}, \underset{\sim}{\sigma^{2}}, p_{1}, \mathcal{D}, \underset{\sim}{z}\right) \sim N\left\{\frac{d_{k} \sigma_{k}^{2}+e_{k}^{2} \sum_{i=1}^{n} \alpha_{i} Z_{i k}}{\sigma_{k}^{2}+e_{k}^{2} \sum_{i=1}^{n} Z_{i k}}, \frac{e_{k}^{2} \sigma_{k}^{2}}{\sigma_{k}^{2}+e_{k}^{2} \sum_{i=1}^{n} Z_{i k}}\right\}$

onde $k \neq \underline{k}, \quad k, \underline{k}=1,2$ 


\section{Capítulo 5}

\section{SELEÇÃO DE MODELOS}

Nos capítulos anteriores apresentamos alguns modelos para modelagem de dados binários correlacionados, e usualmente temos que decidir qual se adequa melhor aos dados.

Para selecionar um modelo, descrevemos dois métodos para discriminação dos mesmos.

O primeiro método baseia-se no erro de previsão do modelo, onde observamos a distância entre os dados observados e dados esperados através do modelo, onde de uma forma empírica, quanto menor for a distância, melhor será o modelo. Para isso fazemos uso dos resíduos de Pearson (ver por exemplo, Dey \& Chen, 1996).

No segundo método a avaliação do modelo passa pela verossimilhança, e como ela provém da distribuição dos dados baseada no modelo é chamada de verossimilhança preditiva ou marginal. Este método considera a razão das verossimilhanças preditivas, que é chamado de fator de Bayes, fornecendo uma medida relativa de avaliação do modelo.

\subsection{Resíduos de Pearson}

$\mathrm{Na}$ estatística clássica é empregado o teste goodness of fit, para verificar a plausividade do modelo ajustado aos dados. O teste clássico goodness of fit quantifica a extremidade de uma medida de discrepância particular calculando uma probabilidade da área sob as extremidades da curva do modelo sob específica hipótese de nulidade.

Como alternativa à estatística clássica goodness of fit, Dey e Chen (1996), desenvolvem um método Bayesiano de checagem de modelos para dados binários correlacionados. 
Para incorporar a natural correlação das respostas binárias, eles introduzem um resíduo de Pearson multivariado para medir a discrepância entre os dados e o modelo, que é definido como

$$
\left(d_{i 1}(\theta), d_{i 2}(\theta), \ldots, d_{i J}(\theta)\right)^{\prime}=\left(\Sigma_{i}^{*}(\theta)\right)^{-1 / 2}\left(y_{i}-p_{i}(\theta)\right)
$$

onde $p_{i}(\theta)=\left(p_{i 1}(\theta), p_{i 2}(\theta), \ldots, p_{i J}(\theta)\right)^{\prime}$ e $\Sigma_{i}^{*}(\theta)$ é a matriz de covariância de $Y_{i}$.

No caso de interesse nas observações individuais, por exemplo, identificar se determinada observação é um possível outlier, o resíduo de Pearson a nível de observação é definido por:

$$
d_{i j}=\frac{\left(y_{i j}-p_{i j}\right)}{\sqrt{p_{i j}\left(1-p_{i j}\right)}} \quad i=1,2, \ldots, n ; j=1,2, \ldots, J .
$$

Além disso para verificar o desempenho global do modelo de interesse, define-se a medida total de discrepância do resíduo de Pearson como (total de Pearson),

$$
D=\sum_{i=1}^{n} \sum_{j=1}^{J} \frac{\left(y_{i j}-p_{i j}\right)^{2}}{p_{i j}\left(1-p_{i j}\right)}
$$

Assim, de forma empírica, quanto menor o valor de $D$ mais apropriado é o modelo.

\subsection{Um Procedimento Bayesiano para Verificação da adequação de Modelos - Fator de Bayes}

O fator de Bayes é uma ferramenta Bayesiana para inferência estatística, e pode ser visto como uma analogia da estatística da razão de verossimilhança.

O fator de Bayes é a razão das verossimilhanças marginais dos dados $y$ sob dois modelos concorrentes $M_{1}$ e $M_{0}$, permitindo assim que se faça uma comparação dos dois modelos. O fator de Bayes $B_{10}$ para o modelo $M_{1}$ contra o modelo $M_{0}$ é a razão dada por,

$$
B_{10}=\frac{p\left(y \mid M_{1}\right)}{p\left(y \mid M_{0}\right)}
$$

Considerando o $k$-ésimo modelo $\left(M_{k}\right)$, a verossimilhança marginal é obtida através da seguinte integral:

$$
p\left(y \mid M_{k}\right)=\int \mathrm{L}\left(y \mid \underset{\sim}{\stackrel{\theta}{\sim}}, M_{k}\right) \pi\left(\underset{\sim}{\theta} \mid M_{k}\right) d \underset{\sim_{k}}{\theta} .
$$

onde 
$\mathrm{E}\left(y \mid \underset{\sim}{\theta}, M_{k}\right)$ é a função de verossimilhança sob o modelo $M_{k}$

$\pi\left(\underset{\sim}{\theta} \mid M_{k}\right)$ é a distribuição a priori para os parâmetros do modelo $M_{k}$

$\underset{\sim}{\theta}$ é o vetor de parâmetros do modelo $M_{k}$.

Indiferente da densidade à priori usada para o modelo em questão, o problema reside no cálculo das integrais que geralmente são intratáveis matematicamente. Assim a solução é fazermos uma aproximação do fator de Bayes através de aproximações das integrais do numerador e do denominador do fator de Bayes. A seguir temos algumas das possíveis aproximações, e como estamos interessados em estimar a integral independente do modelo omitiremos as referências para o modelo $M_{k}$, assim como os $k$ 's subscritos dos $\stackrel{\theta}{\sim}$

\subsubsection{Aproximação de Monte Carlo - (MC)}

Como uma primeira forma de aproximação para integral do tipo

$$
p(y)=\int \mathrm{L}(y \mid \underset{\sim}{\theta}) \pi(\underset{\sim}{\theta}) d \underset{\sim}{\theta},
$$

é a integração de Monte Carlo, que fornece o estimador

$$
\widehat{p}_{M C}(y)=\frac{1}{m} \sum_{t=1}^{m} \mathrm{~L}\left(y \mid \underset{\sim}{\theta^{t}}\right)
$$

onde $\mathrm{E}\left(y \mid \underset{\sim}{\theta^{t}}\right)$ é a verossimilhança de $y,\left\{\underset{\sim}{\theta^{t}}: t=1, \ldots, m\right\}$ são as amostras da densidade $\pi(y)$ e $\pi(\underset{\sim}{\theta})$ é a distribuição a priori. A integração de MC garante a convergência de seu estimador mas esta convergência pode ser lenta. Este resultado se deve a possíveis discrepâncias entre $\mathrm{E}\left(y \mid \underset{\sim}{\theta^{t}}\right)$ e $\pi(\underset{\sim}{\theta})$.

\subsubsection{Aproximações de Monte Carlo em Cadeias de Markov - (MCMC)}

A potencial ineficiência da integração de MC conduz ao desenvolvimento de uma amostragem por importância, na qual a integral (5.4) é escrita por:

$$
p(y)=\int \mathrm{L}(y \mid \underset{\sim}{\theta})\left[\frac{\pi\left(\underset{\sim_{k}}{\theta}\right)}{\left.k \pi *{\underset{\sim}{\theta}}_{k}\right)}\right] k \pi^{*}\left(\underset{\sim_{k}}{\theta}\right) d \underset{\sim_{k}}{\theta},
$$


onde $\mathrm{L}(y \mid \underset{\sim}{\theta})$ e $\pi(\underset{\sim}{\theta})$ novamente, representam a verossimilhança e a distribuição a priori, respectivamente, mas agora a amostra ${\underset{\sim}{t}}^{t}$ é tirada da distribuição $k \pi^{*}(\underset{\sim}{\theta})$, chamada de densidade de importância sendo $k$ a constante normalizadora. Portanto

$$
p(y)=\mathbb{E}\left[\frac{\mathrm{E}(y \mid \underset{\sim}{\operatorname{\theta }}) \pi\left(\underset{\sim_{k}}{\theta}\right)}{k \pi^{*}\left({\underset{\sim}{\sim}}_{k}^{\theta}\right)}\right]
$$

assim,

$$
\widehat{p}_{I S}(y)=\frac{1}{m} \sum_{t=1}^{m} \frac{\pi\left(\stackrel{\theta}{\sim}^{t}\right)}{k \pi^{*}\left({\underset{\sim}{\theta}}^{t}\right)} \mathrm{E}\left(y \mid{\underset{\sim}{\sim}}^{t}\right)
$$

Como $k$ é a constante normalizadora da densidade de importância $k \pi^{*}(\underset{\sim}{\theta}), k$ pode ser escrita como:

$$
k=\int k \pi(\underset{\sim}{\theta}) d \underset{\sim}{\theta}=\int \frac{\pi(\underset{\sim}{\sim})}{\pi^{*}(\underset{\sim}{\theta})} k \pi(\underset{\sim}{\theta}) d \underset{\sim}{\theta}
$$

e um estimador pelo método MCMC amostrando os $\underset{\sim}{\theta}$ da densidade $k \pi^{*}$ é dado por

$$
\widehat{k}=\frac{1}{m} \sum_{t=1}^{m} \frac{\pi\left(\stackrel{(}{\sim}^{t}\right)}{\pi^{*}\left(\stackrel{\theta}{\sim}^{t}\right)}
$$

$\log$,

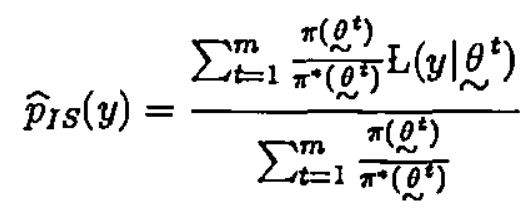

Este estimador para a integral é um peso médio, onde o peso $\pi(\underset{\sim}{\theta}) / \pi^{*}(\underset{\sim}{\theta})$ compensa as os vícios das tiragens incorretas da distribuição, mas a eficiência pode ser ganha amostrando de uma distribuição com uma densidade fechada para o integrando.

Newton e Raftery (1994) propuseram vários estimadores para a verossimilhança marginal baseados em diferentes escolhas de $\pi^{*}(\underset{\sim}{\theta})$.

Algumas formas alternativas:

- O uso da priori $\pi(\underset{\sim}{\theta})$ como função de amostragem de importância $\left(\pi^{*}(\underset{\sim}{\theta})=\pi(\underset{\sim}{\theta})\right)$, leva $\widehat{p}_{I S}(y)$ ao estimador de Monte Carlo, pois $\sum_{t=1}^{m} \frac{\pi(\underbrace{t})}{\pi^{*}\left(\sim^{t}\right)}=m$, assim

$$
\widehat{p}_{1}(y)=\frac{1}{m} \sum_{t=1}^{m} \mathrm{~L}\left(y \mid{\underset{\sim}{\theta^{t}}}^{t}\right)
$$


- $O$ uso da posteriori $\pi(\underset{\sim}{\theta} \mid y)$ como função de amostragem de importância $\left(\pi^{*}(\underset{\sim}{\theta})=\right.$ $\pi(\underset{\sim}{\theta} \mid y))$ leva ao estimador de média harmônica,

$$
\widehat{p}_{2}(y)=\left\{\frac{1}{m} \sum_{t=1}^{m} \Sigma\left(y \mid \underset{\sim}{\theta^{t}}\right)^{-1}\right\}^{-1} .
$$

Este estimador, como $\widehat{p}_{M C}(y)$, é consistente em simulação e é potencialmente mais eficiente porque é principalmente baseado em valores de ${\underset{\sim}{t}}^{t}$ com grande verossimilhança. Entretanto, não satisfaz o teorema do limite central Gaussiano e pode ser instável. Isto porque amostras ocasionais de $\underset{\sim}{\theta^{t}}$ pode ser altamente inconsistentes $\operatorname{com} \mathrm{L}(y \mid \underset{\sim}{\theta})$, e alguns resultados somados $\left(\mathrm{L}\left(y \mid \underset{\sim}{\theta^{t}}\right)\right)^{-1}$ pode dominar o estimador.

- Uma função de importance sampling que é uma mistura das densidades priori e posteriori: $\pi^{*}(\underset{\sim}{\theta})=\delta \pi(\underset{\sim}{\theta})+(1-\delta) \pi(\underset{\sim}{\theta} \mid y)$, onde $0<\delta<1$. Este $\pi^{*}(\underset{\sim}{\theta})$ dá lugar para 0 estimador iterativo

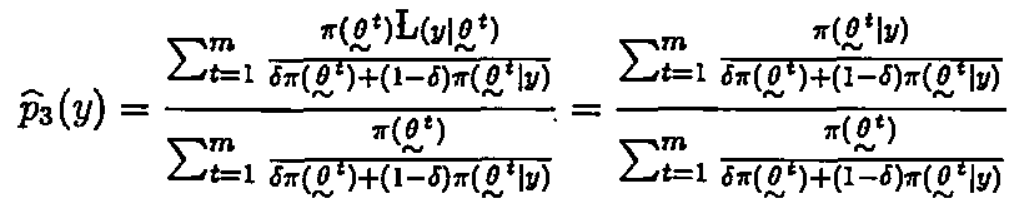

$$
\begin{aligned}
& \widehat{p}_{3}(y)=\frac{\sum_{t=1}^{m} \frac{\mathrm{L}\left(y \mid \underset{\sim}{\left.\right|^{t}}\right)}{\delta \widehat{p}_{3}(y)+(1-\delta) \mathrm{L}\left(y \mid \underset{\sim}{\theta^{t}}\right)}}{\sum_{t=1}^{m} \frac{1}{\delta \widehat{p}_{3}(y)+(1-\delta) \mathrm{L}\left(y \mid \underset{\sim}{\theta^{t}}\right)}}
\end{aligned}
$$

- Newton e Raftery (1994) também consideram uma variação em $\widehat{p}_{2}$ no qual $m$ amostras de $\stackrel{\theta}{\sim}^{t}$ são tiradas da posteriori e $(\delta /(1-\delta) m)$ adicionais amostras imaginárias são tiradas da priori, para qual eles assumiram que a verossimilhança resultante $\mathrm{E}(y \mid \underset{\sim}{\boldsymbol{\theta}})$ é igual a sua expectativa $\pi(y)$. Substituindo o estimador próprio para $\pi(y)$ resulta outro estimador iterativo:

$$
\widehat{p}_{3}(y)=\frac{\frac{\delta m}{1-\delta}+\sum_{t=1}^{m} \frac{\mathrm{L}\left(y \mid \underset{\sim}{\theta^{t}}\right)}{\delta \widehat{p}_{3}(y)+(1-\delta) !\left(y||_{\sim}^{\theta^{t}}\right)}}{\frac{\delta m}{(1-\delta) \widehat{p}_{3}(y)}+\sum_{t=1}^{m} \frac{1}{\delta \widehat{p}_{3}(y)+(1-\delta) \mathrm{L}\left(y \mid \stackrel{\sim}{\sim}^{t}\right)}} .
$$

\subsubsection{A Escolha do Modelo}

Considerando dois modelos, $M_{0}$ e $M_{1}$, temos que o fator de Bayes é dado por:

$$
B_{10}=\frac{p\left(y \mid M_{1}\right)}{p\left(y \mid M_{0}\right)} \text {. }
$$


Para avaliar a evidência de que o modelo $M_{1}$ representa melhor os dados que $M_{0}$, Raftery (1996) apresenta uma calibração do fator de Bayes, considerando a estatística do teste da razão de verossimilhança, que é dada na tabela abaixo.

\begin{tabular}{ccl}
\multicolumn{2}{c}{ Tabela 5.1: Calibração do fator de Bayes } \\
\hline$B_{10}$ & $2 \log B_{10}$ & evidência de $M_{1}$ \\
\hline$<1$ & $<0$ & negativo (apóia $M_{0}$ ) \\
$1 \mapsto 3$ & $0 \mapsto 2$ & vale ser mencionado \\
$3 \mapsto 12$ & $2 \mapsto 5$ & positivo \\
$12 \mapsto 150$ & $5 \mapsto 10$ & forte \\
$>150$ & $>10$ & muito forte \\
\hline \hline
\end{tabular}

Da qual tiramos, que dados dois modelos $M_{i}$ e $M_{j}, i \neq j$, se $p\left(y \mid M_{i}\right)>p\left(y \mid M_{j}\right)$ decidimos pelo modelo $M_{i}$.

Para o caso de $k$ modelos, $M_{1}, M_{2}, \ldots, M_{k}$ decidiremos pelo modelo de maior verossimilhança marginal. 


\section{Capítulo 6}

\section{APLICAÇÕES NUMÉRICAS}

Nos capítulos anteriores descrevemos algumas modelagens para serem aplicadas para dados binários correlacionados. Neste capítulo apresentaremos um exemplo numérico para cada um dos modelos apresentados. Aqui o nosso objetivo é a comparação das diferentes modelagens, utilizando MCMC. Para execução dos programas computacionais utilizamos o software MATLAB 4.2.

\subsection{Ilustração Numérica para o Capítulo 2}

Para ilustrarmos as modelagens do capítulo 2 consideraremos os dados analisados por Huggins (1991), os quais são apresentados abaixo (tabela 1.6), onde as colunas representam sexo (m ou f), a idade (y: jovem, sa: semi-adulto, a: adulto), o peso em gramas, e as observações de captura de 36 animais apanhados em 6 ocasiōes. 
Tabela 6.1: Dados de Huggins

Captura de peromyscus maniculatus coletados por

V. Reid em East Stuart Gulch, Colorado

\begin{tabular}{ccccccccc|ccccccccc}
\hline \hline $\mathrm{m}$ & $\mathrm{y}$ & 12 & 1 & 1 & 1 & 1 & 1 & 1 & $\mathrm{f}$ & $\mathrm{y}$ & 10 & 1 & 0 & 0 & 1 & 0 & 0 \\
$\mathrm{f}$ & $\mathrm{y}$ & 15 & 1 & 0 & 0 & 1 & 1 & 1 & $\mathrm{f}$ & $\mathrm{a}$ & 23 & 0 & 1 & 0 & 0 & 1 & 0 \\
$\mathrm{~m}$ & $\mathrm{y}$ & 15 & 1 & 1 & 0 & 0 & 1 & 1 & $\mathrm{f}$ & $\mathrm{y}$ & 7 & 0 & 1 & 1 & 0 & 0 & 1 \\
$\mathrm{~m}$ & $\mathrm{y}$ & 15 & 1 & 1 & 0 & 1 & 1 & 1 & $\mathrm{~m}$ & $\mathrm{y}$ & 8 & 0 & 1 & 0 & 0 & 0 & 1 \\
$\mathrm{~m}$ & $\mathrm{y}$ & 13 & 1 & 1 & 1 & 1 & 1 & 1 & $\mathrm{~m}$ & $\mathrm{a}$ & 19 & 0 & 1 & 0 & 1 & 0 & 1 \\
$\mathrm{~m}$ & $\mathrm{y}$ & 13 & 0 & 1 & 1 & 0 & 1 & 0 & $\mathrm{f}$ & $\mathrm{a}$ & 22 & 0 & 0 & 1 & 1 & 1 & 1 \\
$\mathrm{f}$ & $\mathrm{y}$ & 5 & 0 & 1 & 0 & 1 & 0 & 1 & $\mathrm{f}$ & $\mathrm{y}$ & 10 & 0 & 0 & 1 & 0 & 1 & 1 \\
$\mathrm{~m}$ & $\mathrm{a}$ & 20 & 0 & 1 & 0 & 0 & 0 & 1 & $\mathrm{f}$ & $\mathrm{y}$ & 14 & 0 & 0 & 1 & 1 & 1 & 1 \\
$\mathrm{~m}$ & $\mathrm{y}$ & 12 & 0 & 1 & 0 & 0 & 1 & 1 & $\mathrm{f}$ & $\mathrm{a}$ & 19 & 0 & 0 & 1 & 0 & 0 & 0 \\
$\mathrm{f}$ & $\mathrm{y}$ & 6 & 0 & 0 & 1 & 0 & 0 & 0 & $\mathrm{f}$ & $\mathrm{a}$ & 20 & 0 & 0 & 0 & 1 & 0 & 0 \\
$\mathrm{~m}$ & $\mathrm{a}$ & 21 & 1 & 1 & 0 & 1 & 1 & 1 & $\mathrm{~m}$ & $\mathrm{sa}$ & 16 & 0 & 0 & 0 & 1 & 1 & 1 \\
$\mathrm{~m}$ & $\mathrm{y}$ & 11 & 1 & 1 & 1 & 1 & 1 & 0 & $\mathrm{f}$ & $\mathrm{y}$ & 11 & 0 & 0 & 0 & 1 & 1 & 0 \\
$\mathrm{~m}$ & $\mathrm{sa}$ & 15 & 1 & 1 & 1 & 0 & 0 & 1 & $\mathrm{~m}$ & $\mathrm{y}$ & 14 & 0 & 0 & 0 & 0 & 1 & 0 \\
$\mathrm{~m}$ & $\mathrm{y}$ & 14 & 1 & 1 & 1 & 1 & 1 & 1 & $\mathrm{f}$ & $\mathrm{y}$ & 11 & 0 & 0 & 0 & 0 & 1 & 0 \\
$\mathrm{~m}$ & $\mathrm{y}$ & 13 & 1 & 1 & 0 & 1 & 1 & 1 & $\mathrm{~m}$ & $\mathrm{a}$ & 24 & 0 & 0 & 0 & 0 & 1 & 0 \\
$\mathrm{f}$ & $\mathrm{a}$ & 22 & 1 & 1 & 1 & 0 & 1 & 1 & $\mathrm{~m}$ & $\mathrm{y}$ & 9 & 0 & 0 & 0 & 0 & 0 & 1 \\
$\mathrm{~m}$ & $\mathrm{y}$ & 14 & 1 & 1 & 1 & 1 & 1 & 1 & $\mathrm{~m}$ & $\mathrm{sa}$ & 16 & 0 & 0 & 0 & 0 & 0 & 1 \\
$\mathrm{~m}$ & $\mathrm{y}$ & 11 & 1 & 0 & 1 & 1 & 1 & 0 & $\mathrm{f}$ & $\mathrm{a}$ & 19 & 0 & 0 & 0 & 0 & 0 & 1 \\
\hline
\end{tabular}

Temos então:

$n=36$ : observaçōes - animais capturados;

$J=6:$ tempos - ocasiões de captura;

$y_{i j}$ : a resposta para a $i$-ésima observação $(i=1,2, \ldots, 36)$ no $j$-ésimo tempo $(j=$ $1,2, \ldots, 6)$, onde:

$y_{i j}= \begin{cases}1 & \text { capturado } \\ 0 & \text { não capturado. }\end{cases}$

$x_{i j}=\left(x_{i j 1}, x_{i j 2}, x_{i j 3}\right):$ o vetor de regressão (covariadas)

Portanto o modelo terá três covariadas, sendo:

$x_{i j 1}= \begin{cases}1 & \text { se é do sexo masculino, } \\ 0 & \text { se é do sexo feminino. }\end{cases}$ 
$x_{i j 2}= \begin{cases}1 & \text { se é jovem, } \\ 2 & \text { se é semi-adulto, } \\ 3 & \text { se é adulto. }\end{cases}$
$x_{i j 3}=($ peso em gramas $)-14$.

Logo o modelo de Prentice(1988) é dado por,

$$
\begin{aligned}
& \theta_{i j}=\frac{\exp \left\{\alpha_{i}+x_{i j 1} \beta_{1 j}+x_{i j 2} \beta_{2 j}+x_{i j 3} \beta_{3 j}\right\}}{1+\exp \left\{\alpha_{i}+x_{i j 1} \beta_{1 j}+x_{i j 2} \beta_{2 j}+x_{i j 3} \beta_{3 j}\right\}} \\
& i=1,2, \ldots, 36 \\
& j=1,2, \ldots, 6
\end{aligned}
$$

onde:

$\alpha_{i}$ : mede a correlação entre as observações ;

$\beta_{1 j}$ : é o efeito da covariável $x_{i j 1}$;

$\beta_{2 j}$ : é o efeito da covariável $x_{i j 2}$;

$\beta_{3 j}$ : é o efeito da covariável $x_{i j 3}$.

De (6.1) temos que a função de verossimilhança (2.5) é dada por,

$$
\mathrm{L}\left(\underset{\sim}{\alpha} \underset{\sim_{1}}{\beta} \underset{\sim_{2}}{\beta}, \underset{\sim_{3}}{\beta} \mid \mathcal{D}\right)=\prod_{i=1}^{36} \prod_{j=1}^{6} \frac{\exp \left\{\left(\alpha_{i}+x_{i j 1} \beta_{1 j}+x_{i j 2} \beta_{2 j}+x_{i j 3} \beta_{3 j}\right) y_{i j}\right\}}{1+\exp \left\{\alpha_{i}+x_{i j 1} \beta_{1 j}+x_{i j 2} \beta_{2 j}+x_{i j 3} \beta_{3 j}\right\}}
$$

onde $\underset{\sim}{\alpha}=\left(\alpha_{1}, \alpha_{2}, \ldots, \alpha_{36}\right)$ e $\mathcal{D}$ representa o conjunto de dados $\left(x_{i j}, y_{i j}\right), i=1, \ldots, 36$; $j=1, \ldots, 6$.

\subsubsection{EMV para os Parâmetros do Modelo com Ligação Logística}

Usando o método da máxima verossimilhança descrito na seçāo 2.1. Temos na tabela 6.2 os estimadores de máxima verossimilhança para os parâmetros do modelo de Prentice (1988), obtidos via método iterativo de Newton-Raphson, e seus respectivos intervalos de confiança (95\%) obtidos por aproximação assintótica. Lembrando que para este caso consideramos efeitos fixos, ou seja, $\alpha_{i}(i=1, \ldots, 36)$ iguais a $\alpha$. 
Tabela 6.2: Estimadores de Máxima Verossimilhança

\begin{tabular}{ccc}
\hline Parâmetro & EMV & Int. Conf. 95\% \\
\hline \hline$\alpha$ & 1.6929 & $(1.4001 ; 1.9858)$ \\
$\beta_{11}$ & 1.3060 & $(0.3655 ; 2.2464)$ \\
$\beta_{12}$ & 0.9334 & $(-0.0468 ; 1.9136)$ \\
$\beta_{13}$ & -0.6831 & $(-1.6009 ; 0.2348)$ \\
$\beta_{14}$ & 0.0606 & $(-0.8408 ; 0.9619)$ \\
$\beta_{15}$ & 1.3575 & $(0.3091 ; 2.4058)$ \\
$\beta_{16}$ & 0.4252 & $(-0.5944 ; 1.4447)$ \\
$\beta_{21}$ & -1.9722 & $(-2.4153 ;-1.5292)$ \\
$\beta_{22}$ & -1.2288 & $(-1.6031 ;-0.8544)$ \\
$\beta_{23}$ & -1.0135 & $(-1.3974 ;-0.6297)$ \\
$\beta_{24}$ & -1.0931 & $(-1.4556 ;-0.7306)$ \\
$\beta_{25}$ & -1.3414 & $(-1.7279 ;-0.9548)$ \\
$\beta_{26}$ & -0.7094 & $(-1.0800 ;-0.3387)$ \\
$\beta_{31}$ & 0.3256 & $(0.1469 ; 0.5043)$ \\
$\beta_{32}$ & 0.1863 & $(0.0428 ; 0.3297)$ \\
$\beta_{33}$ & 0.0876 & $(-0.0556 ; 0.2309)$ \\
$\beta_{34}$ & 0.1560 & $(0.0178 ; 0.2943)$ \\
$\beta_{35}$ & 0.3142 & $(0.1561 ; 0.4723)$ \\
$\beta_{36}$ & 0.1114 & $(-0.0289 ; 0.2518)$ \\
\hline \hline
\end{tabular}

\subsubsection{Aplicação do Modelo 1}

Assumimos efeitos fixos $\left(\alpha_{i}=\alpha ; i=1, \ldots, 36\right)$, para o modelo de Prentice (1988) com as densidades a priori (2.9) e $\mu_{0}=1.6, \sigma_{0}=0.2, b_{11}=1.3, b_{12}=0.9, b_{13}=-0.6$, $b_{14}=0.06, b_{15}=1.3, b_{16}=0.4, b_{21}=-1.9, b_{22}=-1.2, b_{23}=-1.0, b_{24}=-1.0$, $b_{25}=-1.3, b_{26}=-0.7, b_{31}=0.3, b_{32}=0.1, b_{33}=0.08, b_{34}=0.1, b_{35}=0.3, b_{36}=0.1 \mathrm{e}$ $c_{l j}=0.2, l=1,2,3 ; j=1,2, \ldots, 6$.

Para o método de Monte Carlo em Cadeias de Markov, construímos as distribuições condicionadas a posteriori, como na seção (2.3), e a partir delas geramos amostras dos parâmetros.

Como as distribuições condicionadas dos parâmetros não possuem forma padroniza$\mathrm{da}$, as amostras foram geradas através do algoritmo de Metropolis-Hastings, no caso deste algoritmo as amostras geradas podem ter autocorrelação alta. Para diminuir a autocorrelação geramos várias cadeias, com uma grande quantidade de pontos e selecionamos sistematicamente valores de cada cadeia para formar a amostra, ou seja, foram geradas 
5 cadeias de 2000 valores cada, onde cada cadeia inicia com um valor diferente, para a amostra desprezamos os 500 primeiros valores de cada cadeia e selecionamos uma amostra final de 750 valores, tomados de 10 em 10 dos 1500 valores restantes de cada cadeia, isto é, a amostra final é formada pelos valores das iterações : $510^{a}, 520^{a}, \ldots, 2000^{\underline{a}}$ de cada uma das 5 cadeias.

Para o início de cada cadeia foram considerados os seguintes valores: $-5.00 ;-2.75$; $-0.50 ; 1.75 ; 4.00$.

$\mathrm{Na}$ tabela 6.3, apresentamos resumos a posteriori correspondentes aos parâmetros do modelo. Onde encontram-se a média a posteriori, DP o desvio padrão, IC $95 \%$ o intervalo de credibilidade, $\widehat{R}$ o fator de redução de escala do critério de convergência Gelmam e Rubin (ver Apêndice C) e TAc a taxa de aceitação do algoritmo de MetropolisHastings.

Tabela 6.3: Sumário à Posteriori considerando $\alpha_{i}=\alpha$

\begin{tabular}{cccccc}
\hline Parâmetro & Média & DP & IC $95 \%$ & $\sqrt{\widehat{R}}$ & TAc \\
\hline \hline$\alpha$ & 1.6255 & 0.1762 & $(1.2708 ; 1.9756)$ & 1.0011 & 0.6238 \\
$\beta_{11}$ & 1.3177 & 0.2022 & $(0.9157 ; 1.7084)$ & 0.9984 & 0.8971 \\
$\beta_{12}$ & 0.9018 & 0.1942 & $(0.5086 ; 1.2800)$ & 1.0056 & 0.8880 \\
$\beta_{13}$ & -0.5956 & 0.1955 & $(-0.9871 ;-0.2010)$ & 1.0043 & 0.9070 \\
$\beta_{14}$ & 0.0629 & 0.2019 & $(-0.3725 ; 0.4427)$ & 1.0034 & 0.8985 \\
$\beta_{15}$ & 1.3072 & 0.1937 & $(0.8925 ; 1.7308)$ & 1.0004 & 0.9149 \\
$\beta_{16}$ & 0.3999 & 0.2035 & $(0.0150 ; 0.7840)$ & 1.0051 & 0.9100 \\
$\beta_{21}$ & -1.9060 & 0.2082 & $(-2.3076 ;-1.4975)$ & 1.0062 & 0.7535 \\
$\beta_{22}$ & -1.2081 & 0.1968 & $(-1.5937 ;-0.8167)$ & 1.0000 & 0.6974 \\
$\beta_{23}$ & -0.9963 & 0.2028 & $(-1.3845 ;-0.5993)$ & 1.0061 & 0.7254 \\
$\beta_{24}$ & -1.0933 & 0.2083 & $(-1.4968 ;-0.6724)$ & 1.0008 & 0.7062 \\
$\beta_{25}$ & -1.3101 & 0.2044 & $(-1.7068 ;-0.9329)$ & 1.0018 & 0.7330 \\
$\beta_{26}$ & -0.7078 & 0.1965 & $(-1.0747 ;-0.3024)$ & 1.0025 & 0.7135 \\
$\beta_{31}$ & 0.3057 & 0.1993 & $(-0.1013 ; 0.6896)$ & 1.0034 & 0.5000 \\
$\beta_{32}$ & 0.1807 & 0.1992 & $(-0.2258 ; 0.5911)$ & 1.0040 & 0.4162 \\
$\beta_{33}$ & 0.0766 & 0.2089 & $(-0.3093 ; 0.4718)$ & 1.0027 & 0.4365 \\
$\beta_{34}$ & 0.1471 & 0.1922 & $(-0.2292 ; 0.5129)$ & 1.0042 & 0.4165 \\
$\beta_{35}$ & 0.3076 & 0.0965 & $(0.0973 ; 0.5050)$ & 1.0014 & 0.7040 \\
$\beta_{36}$ & 0.1057 & 0.1992 & $(-0.3019 ; 0.4957)$ & 1.0070 & 0.4310 \\
\hline \hline
\end{tabular}

Pela tabela (6.3) verificamos que a taxa de aceitação dos candidatos a parâmetros, gerados pela densidade normal como sendo valores da distribuição a posteriori desejada, estão entre $41.62 \%$ e $91.49 \%$, o que pode ser considerado como alto, indicando que o núcleo 
de transição foi bem escolhido. Pelos valores de $\sqrt{\widehat{R}}$ verificamos que a convergência foi atingida e as amostras selecionadas das iterações são i.i.d., pois $\sqrt{\widehat{R}}<1.1$, portanto o número de iterações é suficiente.

Constatamos que houve a convergência dos valores para uma distribuição de equilíbrio também através dos gráficos que mostram as trajetórias das cadeias geradas ao longo das iterações para cada parâmetro, A seguir damos dois exemplos destes gráficos, e omitimos os demais por conveniência:

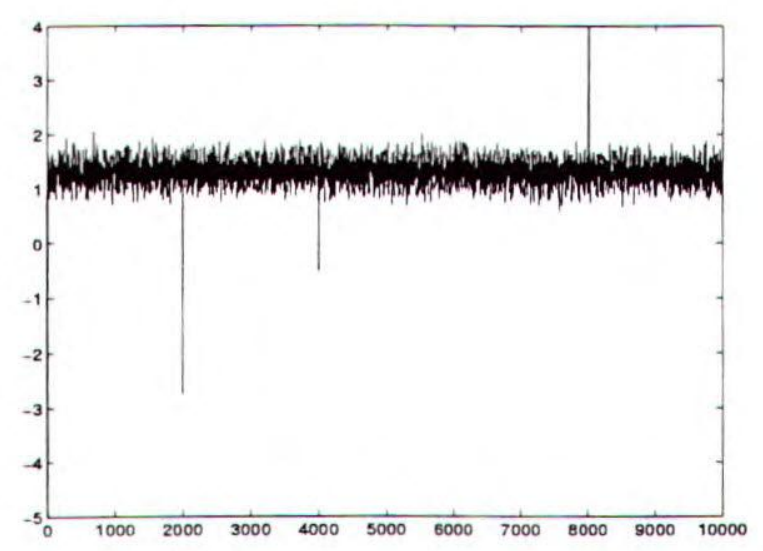

Figura 6.1: Iterações para $\beta_{15}$

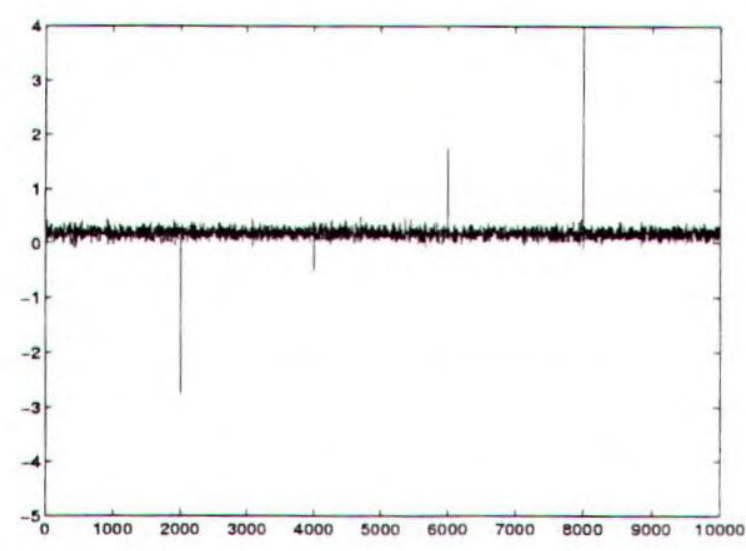

Figura 6.2: Iteraçōes para $\beta_{32}$

Através das figuras de (6.1) e (6.2) podemos observar que após um período inicial, os gráficos apresentam o mesmo comportamento, o que mostra a convergência do algoritmo de Metropolis-Hastings com 5 condições iniciais diferentes para os 19 parâmetros.
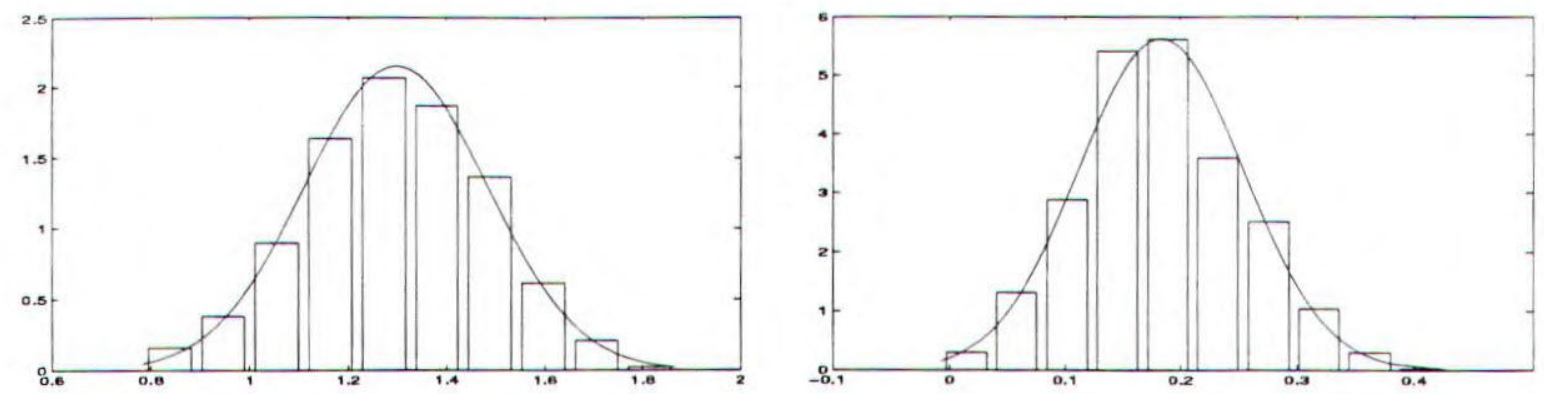

Figura 6.3: Aproximação da densidade Figura 6.4: Aproximação da densidade a a posteriori marginal de $\beta_{15}$ posteriori marginal de $\beta_{32}$

As figuras (6.3) a (6.4) são aproximações das densidades a posteriori marginais dos parâmetros do modelos, obtidas a partir das amostras selecionadas, geradas pelo algoritmo de Metropolis-Hastings. 
Na tabela 6.4 e na figura 6.5 apresentamos os resíduos de Pearson $d_{i j}$ (5.2), que expressa a medida de discrepância entre os dados e o modelo, dado por,

$$
d_{i j}=\frac{y_{i j}-\tilde{p}_{i j}}{\sqrt{\widetilde{p}_{i j} \widetilde{q}_{i j}}},
$$

onde $\widetilde{p}_{i j}$ é $t_{i j}$ dado em (6.1) com os parâmetros estimados por Bayes (tabela 6.3) e $\widetilde{q}_{i j}=$ $1-\tilde{p}_{i j}, i=1,2, \ldots, 36 ; j=1,2, \ldots, 6$.

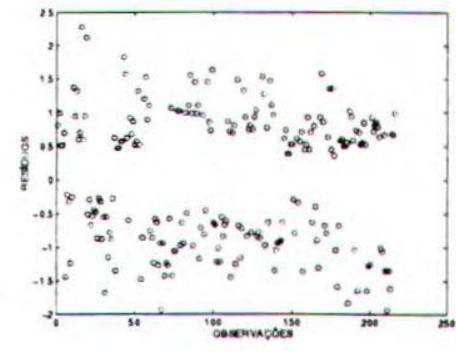

$j=1, \ldots, 6$

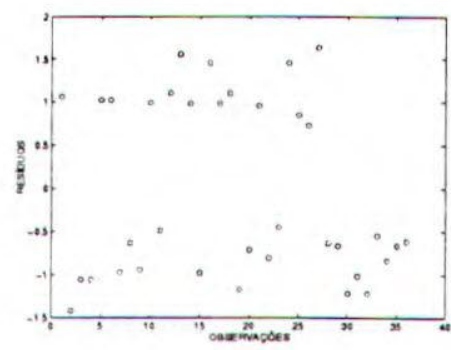

$j=3$

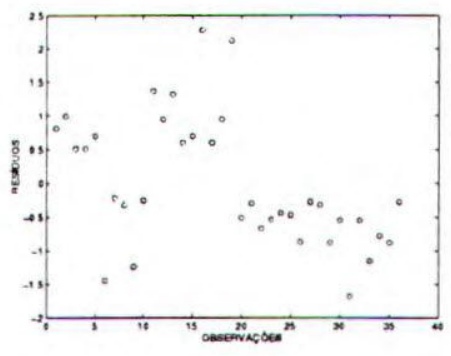

$j=1$

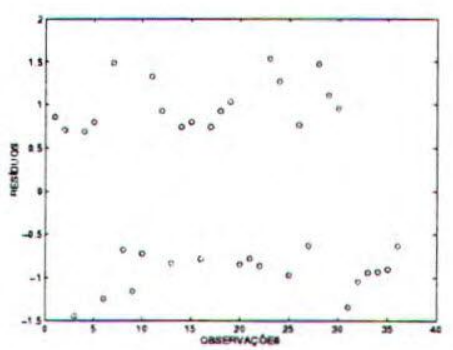

$j=4$

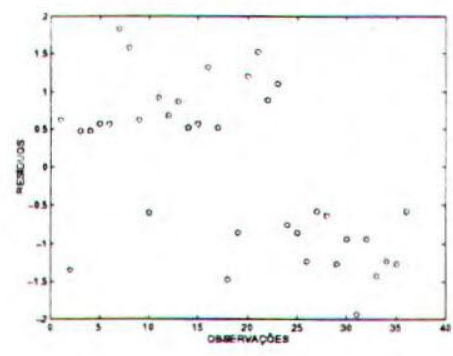

$j=2$

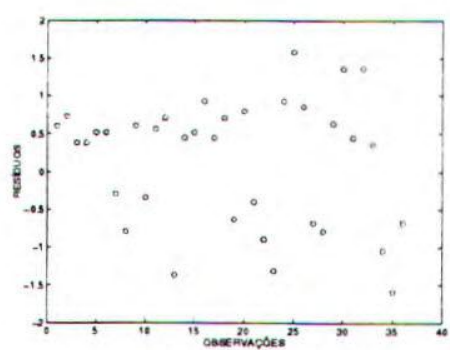

$j=5$

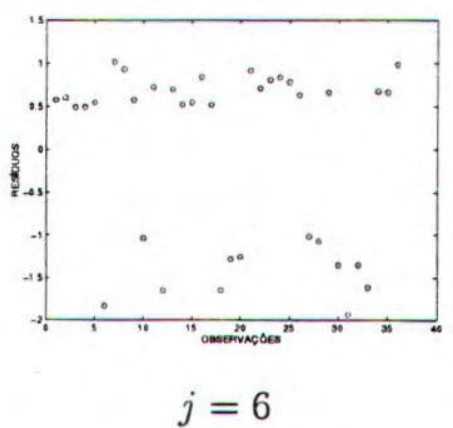

Figura 6.5: Resíduos de Pearson considerando $\alpha_{i}=\alpha(i=1,2, \ldots, 36)$ 
Tabela 6.4: Resíduos de Pearson considerando

$$
\alpha_{i}=\alpha(i=1,2, \ldots, 36)
$$

\begin{tabular}{|c|c|c|c|c|c|}
\hline & & & & & \\
\hline 1 & 2 & 3 & 4 & 5 & 6 \\
\hline 0.8082 & 0.6195 & 1.0616 & 0.8603 & 0.6043 & 0.5752 \\
\hline 0.9875 & -1.3486 & -1.4232 & 0.7120 & 0.7323 & 0.5995 \\
\hline 0.5110 & 0.4724 & -1.0566 & -1.4493 & 0.3809 & 0.4908 \\
\hline 0.5110 & 0.4724 & -1.0566 & 0.6900 & 0.3809 & 0.4908 \\
\hline 0.6937 & 0.5659 & 1.0217 & 0.7993 & 0.5181 & 0.5456 \\
\hline-1.4416 & 0.5659 & 1.0217 & -1.2511 & 0.5181 & -1.8330 \\
\hline-0.2196 & 1.8302 & -0.9703 & 1.4856 & -0.2933 & 1.0169 \\
\hline-0.3233 & 1.5798 & -0.6364 & -0.6798 & -0.7949 & 0.9341 \\
\hline-1.2373 & 0.6195 & -0.9420 & -1.1624 & 0.6043 & 0.5752 \\
\hline-0.2559 & -0.5980 & 0.9918 & -0.72 & & -1.0367 \\
\hline 1.3736 & 0.9195 & -0.4910 & 1.3243 & 0.5611 & 0.7255 \\
\hline 0.9417 & 0.6780 & 1.1031 & 0.9260 & 0.7048 & -1.6491 \\
\hline 1.3252 & 0.8642 & 1.5575 & -0.8 & -1 & 0.6992 \\
\hline 0.5954 & 0. & 0 . & 6 & & \\
\hline 0.6937 & 0.5659 & -0.9787 & 0.7 & 0.5 & 0.5456 \\
\hline 2.2783 & 1.3186 & 1.4554 & -0.7876 & 0.9250 & 0.8404 \\
\hline 0.5954 & 0.5171 & 0.9833 & 0.7426 & 0.4443 & 0.5175 \\
\hline 0.9417 & -1.4748 & 1.1031 & 0.9260 & 0.7 & -1.6491 \\
\hline & & & & & \\
\hline-0.5114 & 1.2047 & -0.7139 & -0.8477 & 31 & -1.2545 \\
\hline-0.2981 & 1.5277 & 0.9546 & -0.7798 & -0.3990 & 0.9149 \\
\hline-0.6713 & 0.8891 & -0.8082 & -0.8661 & -0.8945 & 0.7106 \\
\hline-0.5363 & & -0.4 & & & \\
\hline-0.4389 & -0.7584 & 1.4554 & 1.26 & 0.9250 & 0.8404 \\
\hline-0.4716 & -0.8584 & 0.8510 & -0.9723 & 1.5801 & 0.7808 \\
\hline-0.8691 & -1.2321 & 0.7301 & 0.7664 & 0.8541 & 0.6320 \\
\hline-0.2775 & -0.5783 & 1.6326 & -0.6316 & -0.6815 & -1.0154 \\
\hline-0.3233 & -0.6330 & -0.6364 & 1.4709 & -0.7949 & -1.0705 \\
\hline-0.8792 & -1.2665 & -0.6671 & 1.1074 & 0.6289 & 0.6633 \\
\hline-0.5495 & -0.9396 & -1.2210 & 0.9556 & 1.3549 & -1.3503 \\
\hline-1.6797 & -1.9340 & -1.0169 & -1.3466 & 0.4443 & -1.9325 \\
\hline-0.5495 & -0.9396 & -1.2210 & -1.0465 & 1.3549 & -1.3503 \\
\hline-1.1515 & -1.4262 & -0.5507 & -0.9415 & 0.3537 & -1.6153 \\
\hline-0.7822 & -1.2310 & -0.8397 & -0.9322 & -1.0432 & 0.6740 \\
\hline-0.8792 & -1.2665 & -0.6671 & -0.9030 & -1.5902 & 0.6633 \\
\hline-0.2775 & -0.5783 & -0.6125 & -0.6316 & -0.6815 & 0.9848 \\
\hline
\end{tabular}




\subsubsection{Aplicação do Modelo 2}

Para uma análise Bayesiana assumindo efeitos aleatórios $\alpha_{i}, i=1,2, \ldots, 36$ com uma distribuição $N\left(0, \sigma_{\alpha}^{2}\right)$ com as densidades a priori (2.14) onde $a=4, b=3$ e os demais valores $b_{l j}$ e $c_{l j}$ como sendo os mesmos usados no modelo 1 .

A partir das distribuiçōes condicionais obtidas da distribuição a posteriori (2.11), geramos 5 cadeias de Gibbs, com 6000 iterações cada, descartamos as 3000 primeiras iteraçōes e a partir das 3000 restantes selecionamos com saltos de 10 iteraçōes obtendo uma amostra de 1500 pontos para cada parâmetro.

As quantidades à posteriori de interesse obtidas a partir das amostras selecionadas encontram-se na tabela 6.5, onde observamos que o fator de redução de escala potencial estão bem próxioms de $1.1(\sqrt{\widehat{R}}<1.1)$, indicando a possível convergência das amostras geradas.

Tabela 6.5: Sumário à Posteriori considerando $\alpha_{i} \sim N\left(0, \sigma_{\alpha}^{2}\right)$

\begin{tabular}{crcccc}
\hline Parâmetro & Média & DP & IC 95\% & $\sqrt{\hat{R}}$ & TAc \\
\hline \hline$\sigma_{\alpha}^{2}$ & 0.3267 & 0.2762 & $(0.0568 ; 1.0388)$ & 1.0477 & - \\
$\beta_{11}$ & 1.3715 & 0.1859 & $(0.9998 ; 1.7310)$ & 1.0040 & 0.7899 \\
$\beta_{12}$ & 0.9660 & 0.1874 & $(0.5949 ; 1.3310)$ & 1.0023 & 0.8031 \\
$\beta_{13}$ & -0.5516 & 0.1914 & $(-0.9291 ;-0.1778)$ & 0.9999 & 0.8505 \\
$\beta_{14}$ & 0.1321 & 0.1857 & $(-0.2251 ; 0.4993)$ & 1.0021 & 0.7943 \\
$\beta_{15}$ & 1.3635 & 0.1885 & $(0.9837 ; 1.7359)$ & 1.0015 & 0.8306 \\
$\beta_{16}$ & 0.4574 & 0.1845 & $(0.0845 ; 0.7912)$ & 1.0038 & 0.8183 \\
$\beta_{21}$ & -1.2067 & 0.1614 & $(-1.5263 ;-0.8966)$ & 1.0002 & 0.6757 \\
$\beta_{22}$ & -0.4186 & 0.1560 & $(-0.7417 ;-0.1178)$ & 1.0031 & 0.6549 \\
$\beta_{23}$ & -0.2174 & 0.1552 & $(-0.5212 ; 0.0865)$ & 1.0021 & 0.6697 \\
$\beta_{24}$ & -0.3053 & 0.1529 & $(-0.6073 ;-0.0029)$ & 1.0001 & 0.6253 \\
$\beta_{25}$ & -0.4614 & 0.1603 & $(-0.7772 ;-0.1434)$ & 1.0011 & 0.7206 \\
$\beta_{26}$ & 0.1390 & 0.1558 & $(-0.1690 ; 0.4450)$ & 1.0014 & 0.6733 \\
$\beta_{31}$ & 0.2272 & 0.0926 & $(0.0501 ; 0.4208)$ & 0.9992 & 0.4773 \\
$\beta_{32}$ & 0.0785 & 0.0760 & $(-0.0657 ; 0.2384)$ & 1.0030 & 0.3773 \\
$\beta_{33}$ & -0.0480 & 0.0764 & $(-0.2071 ; 0.0993)$ & 1.0001 & 0.4341 \\
$\beta_{34}$ & 0.0550 & 0.0763 & $(-0.0908 ; 0.2042)$ & 1.0020 & 0.3764 \\
$\beta_{35}$ & 0.2052 & 0.0859 & $(0.0437 ; 0.3867)$ & 0.9998 & 0.3955 \\
$\beta_{36}$ & 0.0056 & 0.0761 & $(-0.1498 ; 0.1538)$ & 0.9993 & 0.3699 \\
\hline \hline
\end{tabular}

A seguir apresentamos os valores (tabela 6.6) e os gráficos (figura 6.6) do resíduo de Pearson para os dados. 
Tabela 6.6: Resíduos de Pearson $\left(\alpha_{i} \sim N\left(0, \sigma_{\alpha}^{2}\right)\right)$

\begin{tabular}{rrrrrr}
\multicolumn{5}{c}{$i=1,2, \ldots, 36$} \\
\hline & 2 & 3 & 4 & 5 & 6 \\
\hline \hline 0.8437 & 0.6005 & 1.0220 & 0.8410 & 0.5709 & 0.5448 \\
1.4171 & -0.9715 & -1.0085 & 0.9841 & 0.9870 & 0.8078 \\
0.7723 & 0.6870 & -0.7074 & -1.0033 & 0.5401 & 0.6953 \\
0.6959 & 0.6191 & -0.7851 & 0.8981 & 0.4867 & 0.6265 \\
0.7601 & 0.5828 & 1.0566 & 0.8258 & 0.5200 & 0.5483 \\
-0.9550 & 0.8028 & 1.4554 & -0.8791 & 0.7163 & -1.3239 \\
-0.2234 & 1.5459 & -1.2640 & 1.3142 & -0.3580 & 0.8426 \\
-0.3214 & 1.4904 & -0.6208 & -0.7411 & -0.9202 & 0.8036 \\
-0.8587 & 0.8289 & -0.7089 & -0.8614 & 0.7880 & 0.7520 \\
-0.2002 & -0.5381 & 1.0132 & -0.6256 & -0.3173 & -0.9519 \\
1.1281 & 0.7129 & -0.5704 & 0.9909 & 0.4000 & 0.5141 \\
1.0574 & 0.6987 & 1.1162 & 0.9671 & 0.7077 & -1.6362 \\
1.3368 & 0.8019 & 1.4920 & -0.9097 & -1.5528 & 0.6141 \\
0.6972 & 0.5758 & 1.1121 & 0.8256 & 0.4822 & 0.5619 \\
0.8517 & 0.6530 & -0.8447 & 0.9253 & 0.5827 & 0.6144 \\
1.8934 & 1.0524 & 1.2908 & -1.0252 & 0.6760 & 0.6103 \\
0.6851 & 0.5658 & 1.0927 & 0.8112 & 0.4738 & 0.5521 \\
1.1602 & -1.3044 & 1.2247 & 1.0611 & 0.7764 & -1.4912 \\
2.9253 & -0.6825 & -0.9720 & 1.3209 & -0.5185 & -1.0436 \\
-0.4263 & 1.4043 & -0.5450 & -0.7593 & 0.8467 & -1.1839 \\
-0.2798 & 1.4323 & 0.8319 & -0.8022 & -0.4386 & 0.8397 \\
-0.5160 & 1.0245 & -0.7386 & -0.7305 & -0.7969 & 0.8034 \\
-0.5857 & 0.9299 & -0.4963 & 1.2625 & -1.6887 & 0.6234 \\
-0.4816 & -0.8666 & 1.4154 & 1.0696 & 0.7413 & 0.6692 \\
-0.3795 & -0.7578 & 0.9266 & -0.8405 & 1.7371 & 0.8631 \\
-0.6442 & -0.9554 & 0.9465 & 0.9891 & 1.0693 & 0.7920 \\
-0.2558 & -0.5753 & 1.7635 & -0.6430 & -0.7406 & -1.1066 \\
-0.2860 & -0.5971 & -0.5525 & 1.5161 & -0.8190 & -1.1075 \\
-0.7515 & -1.1629 & -0.5868 & 1.1927 & 0.6482 & 0.6829 \\
-0.3827 & -0.7093 & -0.9482 & 1.2861 & 1.7419 & -1.0457 \\
-0.8653 & -1.0478 & -0.5425 & -0.7308 & 0.7993 & -1.0737 \\
-0.3432 & -0.6362 & -0.8505 & -0.6974 & 1.9421 & -0.9379 \\
-0.7825 & -0.9907 & -0.3333 & -0.6881 & 0.4683 & -1.2315 \\
-0.5165 & -0.9071 & -0.6444 & -0.6709 & -0.7890 & 0.8966 \\
-0.9441 & -0.4764 & -0.6807 & -1.2525 & 0.8412 \\
& -0.5745 & -0.5662 & -0.6420 & -0.7395 & 0.9050 \\
\hline & & & & &
\end{tabular}




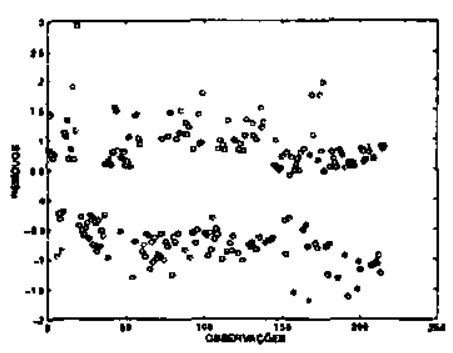

$j=1, \ldots, 6$

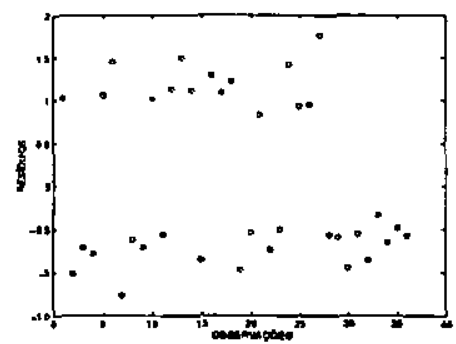

$j=3$

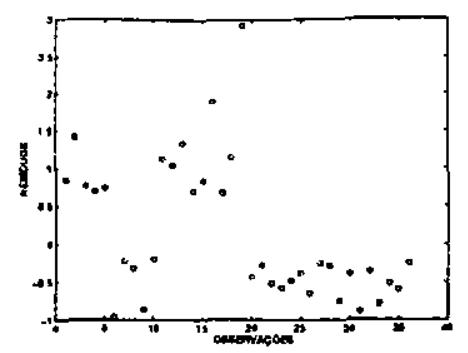

$j=1$

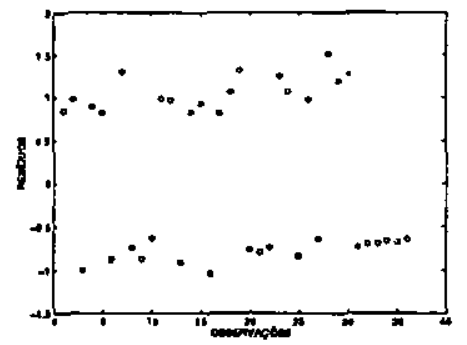

$j=4$

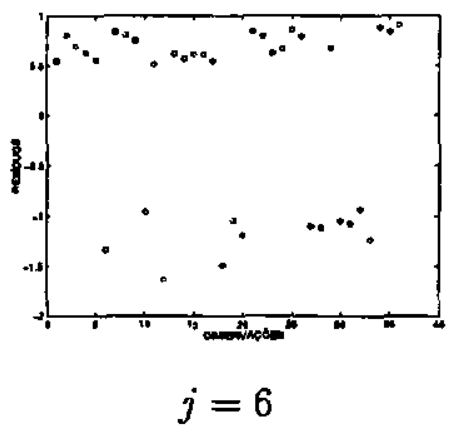

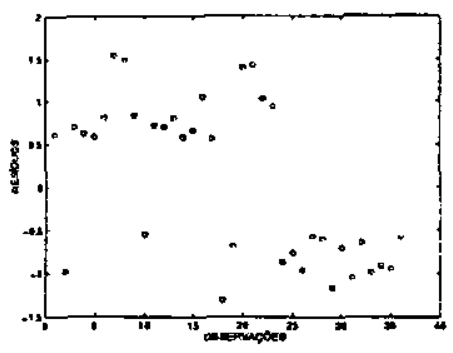

$j=2$

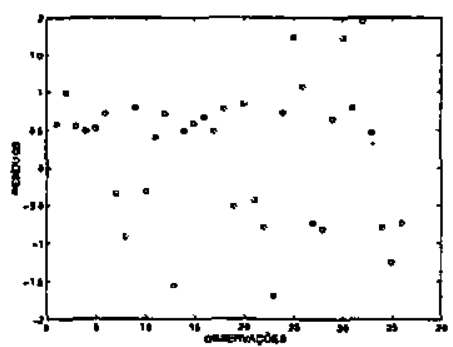

$j=5$

Figura 6.6: Resíduos de Pearson considerando $\alpha_{i} \sim N\left(0, \sigma_{\alpha}^{2}\right), i=1,2, \ldots, 36$

\subsubsection{Aplicação do Modelo 3}

Assumimos agora uma mistura de duas distribuições normais (2.17) para os efeitos aleatórios $\alpha_{i}, i=1,2, \ldots, 36$ e distribuições a priori (2.18) com $d_{1}=-0.4, d_{2}=0.4$, $e_{1}=e_{2}=0.2, a_{1}=a_{2}=4, b_{1}=b_{2}=3, f=1, g=1$ e os valores para $b_{l j}$ e $c_{l j}$ os mesmos usados para o modelo 1 .

$\mathrm{Na}$ tabela 6.7, apresentamos os resumos a posteriori correspondentes ao modelo. Os resultados foram obtidos pelo método MCMC a partir das distribuições condicionais da distribuição a posteriori (2.2), das quais geramos 5 cadeias de 6000 iterações cada e para a amostra, desprezamos as 3000 iterações e a partir deste ponto tomamos de $10 \mathrm{em}$ 10 obtendo assim uma amostra final de 1500 pontos amostrais. 
Tabela 6.7: Sumário à Posteriori considerando $\alpha_{i}$ Mistura de Normais

\begin{tabular}{crcccc}
\hline Parâmetro & Média & DP & IC $95 \%$ & $\sqrt{\widehat{R}}$ & TAc \\
\hline \hline$\mu_{1}$ & -0.6478 & 0.3881 & $(-1.4215 ; 0.1078)$ & 1.0287 & - \\
$\mu_{2}$ & 1.0367 & 0.2925 & $(0.5044 ; 1.6452)$ & 1.0366 & - \\
$\sigma_{1}^{2}$ & 0.1317 & 0.1172 & $(0.0380 ; 0.4470)$ & 1.0147 & - \\
$\sigma_{2}^{2}$ & 0.1308 & 0.0987 & $(0.0381 ; 0.3955)$ & 1.0088 & - \\
$p_{1}$ & 0.3586 & 0.1477 & $(0.0890 ; 0.6551)$ & 1.0289 & - \\
$\beta_{11}$ & 1.2975 & 0.1948 & $(0.9284 ; 1.6791)$ & 1.0014 & 0.6664 \\
$\beta_{12}$ & 0.8999 & 0.1990 & $(0.5140 ; 1.2726)$ & 1.0010 & 0.9015 \\
$\beta_{13}$ & -0.6039 & 0.2042 & $(-1.0033 ;-0.2117)$ & 1.0011 & 0.8766 \\
$\beta_{14}$ & 0.0577 & 0.1934 & $(-0.3100 ; 0.4333)$ & 1.0007 & 0.8829 \\
$\beta_{15}$ & 1.2930 & 0.1964 & $(0.9037 ; 1.6887)$ & 0.9999 & 0.6929 \\
$\beta_{16}$ & 0.3962 & 0.2001 & $(-0.0247 ; 0.7842)$ & 1.0011 & 0.8335 \\
$\beta_{21}$ & -1.2995 & 0.2061 & $(-1.7026 ;-0.8977)$ & 1.0000 & 0.8500 \\
$\beta_{22}$ & -0.5030 & 0.2001 & $(-0.8949 ;-0.1181)$ & 1.0016 & 0.8457 \\
$\beta_{23}$ & -0.3006 & 0.2015 & $(-0.7041 ; 0.0911)$ & 1.0052 & 0.8393 \\
$\beta_{24}$ & -0.3952 & 0.2032 & $(-0.7832 ;-0.0161)$ & 1.0017 & 0.8764 \\
$\beta_{25}$ & -0.5020 & 0.1972 & $(-0.8984 ;-0.0960)$ & 1.0045 & 0.8310 \\
$\beta_{26}$ & 0.0599 & 0.1961 & $(-0.3277 ; 0.4368)$ & 1.0045 & 0.7898 \\
$\beta_{31}$ & 0.3047 & 0.2019 & $(-0.0871 ; 0.7049)$ & 1.0019 & 0.8965 \\
$\beta_{32}$ & 0.1725 & 0.2024 & $(-0.2293 ; 0.5590)$ & 1.0022 & 0.7187 \\
$\beta_{33}$ & -0.0824 & 0.2047 & $(-0.4873 ; 0.3255)$ & 1.0033 & 0.8393 \\
$\beta_{34}$ & 0.1416 & 0.1938 & $(-0.2369 ; 0.5188)$ & 1.0018 & 0.7888 \\
$\beta_{35}$ & 0.3078 & 0.1909 & $(-0.0688 ; 0.7014)$ & 1.0024 & 0.7184 \\
$\beta_{36}$ & 0.1159 & 0.2024 & $(-0.2681 ; 0.5214)$ & 1.0031 & 0.8886 \\
\hline \hline & & & & &
\end{tabular}

Observamos que houve a convergência dos valores para distribuição de equilíbrio através do fator de redução e escala de Gelman-Rubin (1992), que constam na tabela 6.7, onde para todos os parâmetros constatamos que $\sqrt{\widehat{R}}<1.1$. Além disso observamos os gráficos das iterações de todos os parâmetros os quais não se encotram nesta dissertação. Observamos também uma excelente taxa de aceitação dos valores gerados pelo algoritmo Metropolis-Hastings .

A seguir apresentamos a tabela 6.8 com o resíduo de Pearson para cada observação. 
Tabela 6.8: Resíduos de Pearson (mistura de duas distribuições normais para $\alpha_{i}$ )

\begin{tabular}{|c|c|c|c|c|c|}
\hline & & & & & \\
\hline 1 & 2 & 3 & 4 & 5 & 6 \\
\hline 0.7292 & 34 & 7.75 & 326 & 0.4920 & 0.4802 \\
\hline 1.0291 & & & & 0.6896 & \\
\hline 0.6363 & 0.5569 & -0.8247 & -1.2248 & 0.4274 & 0.5561 \\
\hline 0.5220 & 0.4568 & -1.0054 & 0.6698 & 0.3506 & 0.4562 \\
\hline 0.6319 & 0.4845 & 0.8176 & 0.6887 & 0.4257 & 0.4572 \\
\hline-1.0433 & 0.7350 & 1.2402 & -0.9571 & 0.6457 & -1.4417 \\
\hline-0.2121 & 1.7467 & - 1.9948 & 1.4402 & -0.3116 & 1.0218 \\
\hline-0.3549 & 1.2684 & -0.4972 & -0.8447 & -1.1849 & 0.6461 \\
\hline-0.9246 & 0.7763 & -0.8671 & -0.9203 & 0.7298 & 0.7122 \\
\hline-0.1507 & -0.3809 & 0.8559 & -0.4549 & -0.2218 & -0.6329 \\
\hline 0.7499 & 0.4399 & -0.5949 & 0.6354 & 0.2 & 0.2966 \\
\hline 0.9131 & 5 & 0.8023 & 0.8 & 0.6171 & -1.8279 \\
\hline 1.0799 & 6 & 1.2488 & -1.1343 & -2.0543 & 0.4783 \\
\hline 0.5442 & 0.4458 & 0.8546 & 0.6436 & 0.3661 & 0.4328 \\
\hline 0.6951 & 0.5330 & -1.1118 & 0.7577 & 0.4683 & 0.5030 \\
\hline 1.2183 & 0.6259 & 1.2808 & -1.6596 & 0.3638 & 0.3374 \\
\hline 0.5427 & 0.4446 & 0.8522 & 0.6419 & 0.3651 & 0.4316 \\
\hline 1.0104 & -1.4732 & 0.8877 & 0.9356 & 0.6828 & -1.6519 \\
\hline 2.8697 & -0.6760 & -1.2454 & 1.3177 & -0.5160 & -1.0031 \\
\hline-0.4908 & 1.1184 & -0.3847 & -0.9146 & 0.6075 & -1.6124 \\
\hline-0.2847 & 1.4847 & 0.5498 & -0.7920 & -0.4197 & 0.9191 \\
\hline-0.4560 & 1.2 & -0.9275 & -0.6289 & -0.6716 & 0.9900 \\
\hline-0.6897 & 53 & -0.4531 & 1.0436 & -2.2939 & 0.4748 \\
\hline-0.7434 & -1.4469 & 1.4143 & 0.6653 & 0.4017 & 0.3726 \\
\hline-0.4361 & -0.8460 & 0.6416 & -0.9498 & 1.5486 & 0.7966 \\
\hline-0.8355 & -1.2442 & 0.7264 & 0.7615 & 0.8033 & 0.6066 \\
\hline-0.2595 & -0.6159 & 2.2669 & -0.6701 & -0.8650 & -1.2437 \\
\hline-0.2682 & -0.5959 & -0.3757 & 1.5664 & -0.8955 & -1.1698 \\
\hline-0.8469 & -1.3490 & -0.6035 & 1.0459 & 0.5314 & 0.5748 \\
\hline-0.4004 & -0.7270 & -1.1790 & 1.2443 & 1.6 & -1.0486 \\
\hline-0.7195 & -0.8784 & -0.4582 & -0.6084 & 0.9349 & -0.9047 \\
\hline-0.2882 & -0.5233 & -0.8487 & -0.5785 & 2.3399 & -0.7548 \\
\hline-0.8582 & -1.1996 & -0.2142 & -0.7930 & 0.3477 & -1.6348 \\
\hline-0.4268 & -0.7251 & -0.7154 & -0.5426 & .0.6296 & 1.1622 \\
\hline-0.5324 & -0.8479 & -0.3793 & -0.6010 & -1.1828 & 0.9144 \\
\hline-0.2380 & -0.5648 & -0.4045 & -0.6145 & -0.7933 & 0.8768 \\
\hline
\end{tabular}

A figura 6.7 apresenta a tabela em forma de gráfico, primeiramente com todos os tempos e em seguida um gráfico para cada tempo 


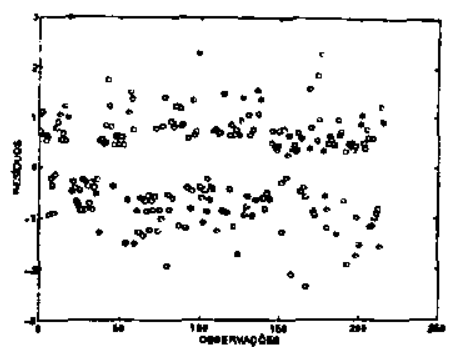

$j=1, \ldots, 6$

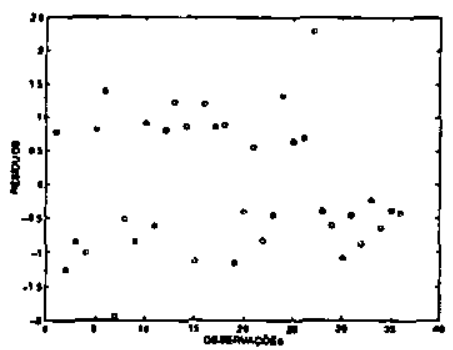

$j=3$

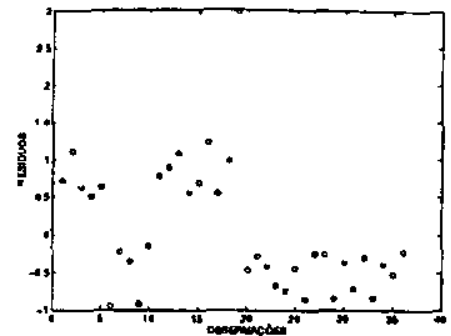

$j=1$

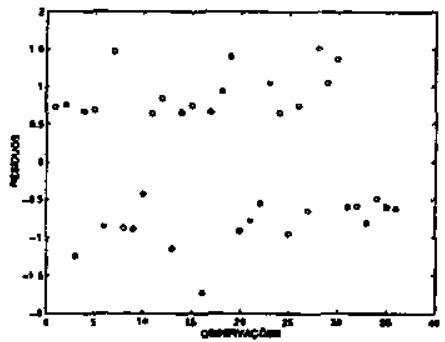

$j=4$

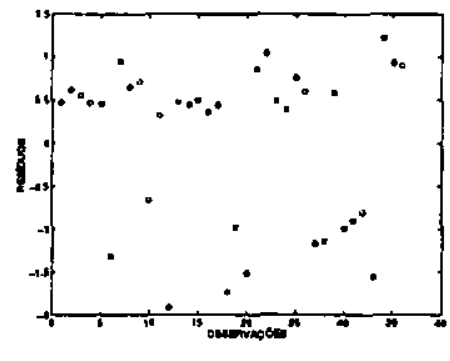

$j=6$

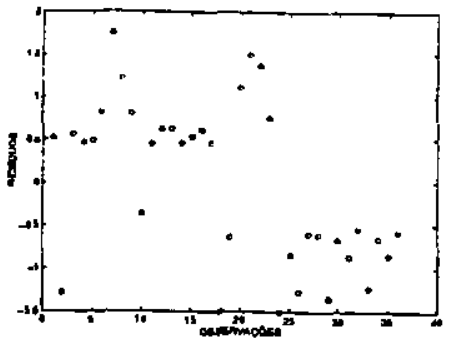

$j=2$

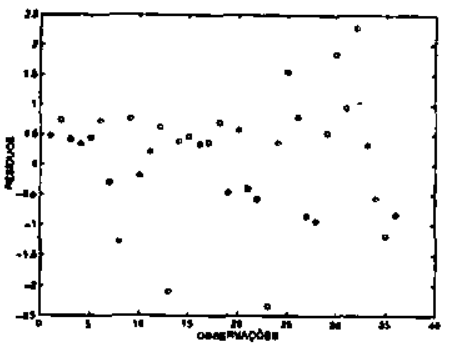

$j=5$

Figura 6.7: Resíduo de Pearson assumindo o mistura de normais para os efeitos aleatórios

\subsubsection{Comparação dos Modelos 1, 2 e 3}

Na tabela 6.9, temos um resumo dos estimadores de Monte Carlo para a média a posteriori dos parâmetros $\beta_{l j}(l=1,2,3 ; j=1,2, \ldots, 6)$ baseados em amostras de Metropolis-Hastings considerando os diferentes modelos com função de ligação logística (modelo 1, modelo 2 e modelo 3 ). 
Tabela 6.9: Médias a posteriori para $\beta_{l j}(l=1,2,3 ; j=1,2, \ldots, 6)$

\begin{tabular}{ccccc}
\hline Parâmetro & EMV & $\alpha$ Fixo & $\alpha_{i} \sim N\left(0, \sigma_{\alpha}^{2}\right)$ & $\alpha_{i} \sim$ Mistura de Normais \\
\hline \hline$\beta_{11}$ & 1.3060 & 1.3177 & 1.3715 & 1.2975 \\
$\beta_{12}$ & 0.9334 & 0.9018 & 0.9660 & 0.8999 \\
$\beta_{13}$ & -0.6831 & -0.5956 & -0.5516 & -0.6039 \\
$\beta_{14}$ & 0.0606 & 0.0629 & 0.1321 & 0.0577 \\
$\beta_{15}$ & 1.3575 & 1.3072 & 1.3635 & 1.2930 \\
$\beta_{16}$ & 0.4252 & 0.3999 & 0.4574 & 0.3962 \\
$\beta_{21}$ & -1.9722 & -1.9060 & -1.2067 & -1.2995 \\
$\beta_{22}$ & -1.2288 & -1.2081 & -0.4186 & -0.5030 \\
$\beta_{23}$ & -1.0135 & -0.9963 & -0.2174 & -0.3006 \\
$\beta_{24}$ & -1.0931 & -1.0933 & -0.3053 & -0.3952 \\
$\beta_{25}$ & -1.3414 & -1.3101 & -0.4614 & -0.5020 \\
$\beta_{26}$ & -0.7094 & -0.7078 & 0.1390 & 0.0599 \\
$\beta_{31}$ & 0.3256 & 0.3057 & 0.2272 & 0.3047 \\
$\beta_{32}$ & 0.1863 & 0.1807 & 0.0785 & 0.1725 \\
$\beta_{33}$ & 0.0876 & 0.0766 & -0.0480 & -0.0824 \\
$\beta_{34}$ & 0.1560 & 0.1471 & 0.0550 & 0.1416 \\
$\beta_{35}$ & 0.3142 & 0.3076 & 0.2052 & 0.3078 \\
$\beta_{36}$ & 0.1114 & 0.1057 & 0.0056 & 0.1159 \\
\hline \hline
\end{tabular}

Podemos observar nas tabelas 6.3, 6.5 e principalmente na tabela 6.7 a partir dos intervalos de credibilidade $95 \%$ para os parâmetros de regressão $\beta_{l j}, l=1,2,3 ; j=$ $1,2, \ldots, 6$ que as covariáveis $x_{i j 1}$ (sexo) e $x_{i j 2}$ (idade) $i=1,2, \ldots, 36 ; j=1,2, \ldots, 6$ apresentam efeitos significativos na probabilidade de captura nos diferentes tempos, mas a covariável $x_{i j 3}$ (peso) não apresenta efeito significativo na probabilidade de captura, pois os intervalos de credibilidade para $\beta_{3 j}, j=1,2, \ldots, 6$ incluem zero.

Verificamos que nas tabelas 6.6 e 6.8 que a $19^{a}$ observação tem o valor resíduo de Pearson $d_{i j}$ alto, sendo um possível outlier em específico para $j=1$, isto fica mais visível quando observamos as figuras 6.6 e 6.7 para $j=1$. Então na tentativa de melhorarmos os modelos retiramos a $19^{a}$ observação do conjuto de dados e obtivemos novos estimadores para os parâmetros dos modelos. Na tabela 6.10 verificarmos a performance dos modelos para os dados com e sem o outlier (conjunto de dados com 36 e 35 obserções), através do Total de Pearson (5.3),

$$
D=\sum_{i=1}^{n} \sum_{j=1}^{J} \frac{\left(y_{i j}-\widetilde{p}_{i j}\right)^{2}}{\widetilde{\tilde{p}}_{i j}\left(1-\widetilde{p}_{i j}\right)}
$$

onde $\widetilde{p}_{i j}$ é $t_{i j}$ dado em (6.1) com os parâmetros estimados por Bayes e $\widetilde{q}_{i j}=1-\widetilde{p}_{i j}$, $i=1,2, \ldots, 36 ; j=1,2, \ldots, 6$. Também apresentamos a verossimilhança preditiva (5.9), 
dada por,

$$
\begin{aligned}
& \widehat{p}_{2}\left(\widehat{y} \mid M_{k}\right)=\left\{\frac{1}{m} \sum_{t=1}^{m} \mathrm{E}\left(d \mid \underset{\sim}{\alpha^{(t)}} \underset{\sim_{1}}{\beta^{(t)}} \underset{\sim_{2}}{\beta^{(t)}}, \underset{\sim_{3}}{\beta^{(t)}}\right)^{-1}\right\}^{-1} \\
& =\left\{\frac{1}{m} \sum_{t=1}^{m}\left[\prod_{i=1}^{36} \prod_{j=1}^{6} \frac{\exp \left\{\left(\alpha_{i}^{(t)}+x_{i j 1} \beta_{1 j}^{(t)}+x_{i j 2} \beta_{2 j}^{(t)}+x_{i j 3} \beta_{3 j}^{(t)}\right) y_{i j}\right\}}{1+\exp \left\{\alpha_{i}^{(t)}+x_{i j 1} \beta_{1 j}^{(t)}+x_{i j 2} \beta_{2 j}^{(t)}+x_{i j 3} \beta_{3 j}^{(t)}\right\}}\right]^{-1}\right\}^{-1} \\
& i=1,2, \ldots, n ; \quad j=1,2, \ldots, 6 ; \quad k=1,2,3 \text {; }
\end{aligned}
$$

onde $k$ indica o modelo o qual esta sendo calculada a densidade preditiva e $m$ o tamanho da amostra gerada.

Tabela 6.10: Resultados do Total de Pearson (6.4) e as Densidades Preditivas para os Modelos

\begin{tabular}{c|ccc}
\hline & Modelo 1 & Modelo 2 & Modelo 3 \\
\hline \hline$D(36$ obs $)$ & 207.5838 & 185.7211 & 183.3444 \\
$D(35$ obs $)$ & 198.2011 & 177.0421 & 175.0432 \\
$\widehat{p}_{2}\left(\widehat{y} \mid M_{k}\right)(36$ obs $)$ & $5.3519 \cdot 10^{-84}$ & $2.9348 \cdot 10^{-61}$ & $6.7799 \cdot 10^{-59}$ \\
$\widehat{p}_{2}\left(\widehat{y} \mid M_{k}\right)(35 \mathrm{obs})$ & $9.6933 \cdot 10^{-77}$ & $3.0310 \cdot 10^{-59}$ & $6.2787 \cdot 10^{-57}$ \\
\hline \hline
\end{tabular}

Observamos através da tabela 6.10 que para os dados binários correlacionados da tabela 6.1, considerando a função de ligação logística, o modelo de regressão logístico de efeitos aleatórios, considerando mistura de duas distribuições normais, mostra-se mais adequado. Isso também se verifica pela densidade preditiva, observando a tabela 5.1 de calibração do fator de Bayes. Podemos considerar uma forte evidência de que o modelo 3 é o mais adequado.

Além da análise dos dados que apresentamos aqui, também consideramos a possibilidade de haver interações entre as covariáveis com e sem o ponto considerado outlier, mas os resultados não foram significativos, pois os intervalos de credibilidade para estes novos parâmetros incluiam zero, e também houve um sensível aumento no Total de Pearson.

As figuras 6.8 e 6.9 apresentam o histograma das frequências (Fr) dos valores de $\alpha_{i}, i=1,2, \ldots, 36$ para o modelo 2 e o modelo 3, respectivamente. Através dos gráficos podemos verificar que modelar estes parâmetros por uma única distribuição pode não ser apropriado. 


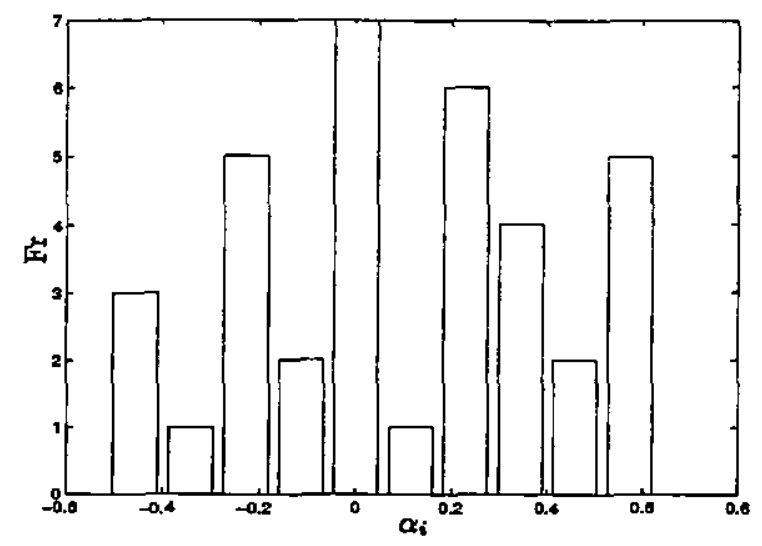

Figura 6.8: Frequências dos $\alpha_{i}$ Assumindo $\alpha_{i} \stackrel{i j d}{\sim} N\left(0, \sigma_{\alpha}^{2}\right) i=1,2, \ldots, 36$

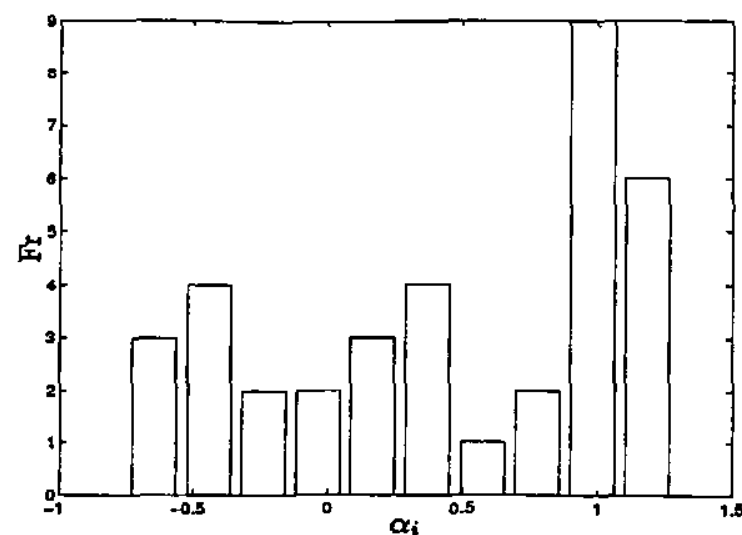

Figura 6.9: Frequências dos $\alpha_{i}$ Assumindo $\alpha_{i}, i=1,2, \ldots, 36$ Mistura de duas Normais

\subsection{Ilustração Numérica para o Capítulo 3}

Para exemplificar o capítulo 3 também usamos o conjunto de dados apresentados por Huggins (1991), dados na tabela 6.1.

Sem considerar interações entre as covariáveis a função de ligação probito para os dados da tabela 6.1 será dada por,

$$
\begin{aligned}
\theta_{i j}=\Phi\left\{\alpha_{i}+x_{i j 1} \beta_{1 j}+x_{i j 2} \beta_{2 j}+x_{i j 3} \beta_{3 j}\right\}, \quad & i=1,2, \ldots, 36 \\
& j=1,2, \ldots, 6
\end{aligned}
$$

onde:

$\alpha_{i}$ : mede a correlação entre as observações ;

$\beta_{1 j}$ : é o efeito da covariável $x_{i j 1}$;

$\beta_{2 j}$ : é o efeito da covariável $x_{i j 2}$;

$\beta_{3 j}$ : é o efeito da covariável $x_{i j 3}$.

De (6.6), a função de verossimilhança (3.2) é dada por,

$$
\begin{aligned}
\mathrm{L}\left(\underset{\sim}{\alpha}, \beta_{1}, \beta_{2}, \ldots, \beta_{J} \mid \mathcal{D}\right)=\prod_{i=1}^{36} \prod_{j=1}^{6} \Phi\left(\alpha_{i}+x_{i j 1} \beta_{1 j}+x_{i j 2} \beta_{2 j}+x_{i j 3} \beta_{3 j}\right)^{y_{i j}} \\
{\left[1-\Phi\left(\alpha_{i}+x_{i j 1} \beta_{1 j}+x_{i j 2} \beta_{2 j}+x_{i j 3} \beta_{3 j}\right)\right]^{1-y_{i j}} }
\end{aligned}
$$

onde $\underset{\sim}{\alpha}=\left(\alpha_{1}, \alpha_{2}, \ldots, \alpha_{36}\right)$ e $\mathcal{D}$ representa o conjunto de dados $\left(x_{i j}, y_{i j}\right), i=1, \ldots, 36$ $j=1, \ldots, 6$. 


\subsubsection{EMV para os Parâmetros do Modelo com Ligação Probito}

Nesta seção, considerando os dados da tabela 6.1, aplicamos a metodologia apresentada na seção 3.1 de Análise Clássica, baseada na aproximação Normal assintótica dos estimadores de máxima verossimilhança, dos parâmetros envolvidos no modelo considerando efeitos fixos, $\alpha_{i}, i=1,2, \ldots, 36$ iguais à $\alpha$.

A partir do método iterativo de Newton-Raphson obtemos os estimadores de máxima verossimilhança para os parâmetros, juntamente com os respectivos intervalos de confiança assintóticos $95 \%$ descritos na tabela 6.11

Tabela 6.11: Estimadores de Máxima Verossimilhança

\begin{tabular}{ccc}
\hline Parâmetro & EMV & Int. Conf. 95\% \\
\hline \hline$\alpha$ & 1.0629 & $(0.8853 ; 0.8853)$ \\
$\beta_{11}$ & 0.7799 & $(0.2052 ; 0.2052)$ \\
$\beta_{12}$ & 0.5650 & $(-0.0220 ;-0.0220)$ \\
$\beta_{13}$ & -0.4330 & $(-1.0024 ;-1.0024)$ \\
$\beta_{14}$ & 0.0336 & $(-0.5209 ;-0.5209)$ \\
$\beta_{15}$ & 0.8677 & $(0.2285 ; 0.2285)$ \\
$\beta_{16}$ & 0.2403 & $(-0.3548 ;-0.3548)$ \\
$\beta_{21}$ & -1.2116 & $(-1.4671 ;-1.4671)$ \\
$\beta_{22}$ & -0.7623 & $(-0.9872 ;-0.9872)$ \\
$\beta_{23}$ & -0.6351 & $(-0.8679 ;-0.8679)$ \\
$\beta_{24}$ & -0.6830 & $(-0.9057 ;-0.9057)$ \\
$\beta_{25}$ & -0.8444 & $(-1.0842 ;-1.0842)$ \\
$\beta_{26}$ & -0.4452 & $(-0.6713 ;-0.6713)$ \\
$\beta_{31}$ & 0.2012 & $(0.0991 ; 0.0991)$ \\
$\beta_{32}$ & 0.1129 & $(0.0289 ; 0.0289)$ \\
$\beta_{33}$ & 0.0549 & $(-0.0333 ;-0.0333)$ \\
$\beta_{34}$ & 0.0973 & $(0.0125 ; 0.0125)$ \\
$\beta_{35}$ & 0.1968 & $(0.0987 ; 0.0987)$ \\
$\beta_{36}$ & 0.0698 & $(-0.0148 ;-0.0148)$ \\
\hline \hline
\end{tabular}

\subsubsection{Aplicação do Modelo 4}

Considerando os dados introduzidos por Huggins (1991), e o modelo probito de efeitos fixos, escolhemos os seguintes valores para as densidades a priori: $\mu_{0}=1, \sigma_{0}^{2}=$ $0.4, b_{11}=0.7, b_{12}=0.5, b_{13}=-0.4, b_{14}=0.03, b_{15}=0.8, b_{16}=0.2, b_{21}=-1.2$, $\left.b_{22}=-0.7, b_{23}=-0.6, b_{24}=-0.6, b_{25}=-0.8, b_{26}=-0.4\right], b_{31}=0.2, b_{32}=0.1$, $b_{33}=0.05, b_{34}=0.09, b_{35}=0.19, b_{36}=0.06$ e $c_{k j}=0.4, k=1,2,3 ; j=1,2, \ldots, 6$. 
Na tabela 6.12, apresentamos os resumos a posteriori correspondentes aos parâmetros do modelo 4, a partir das distribuiçōes condicionais das quais geramos 5 cadeias de Metropolis-Hastings de 6000 amostras cada, todas as cadeias com condições iniciais diferentes, desprezamos as 3000 primeiras iteraçōes, a partir destas tomamos a $3010^{\underline{a}}, 3020^{a}, \ldots$ a fim de dimimuir a auto correlação das amostras, formando assim amostras de tamanho 1500 para cada parâmetro. Podemos considerar a convergência para as distribuições de equilíbrio, pois pela tabela $6.12, \sqrt{\widehat{R}}<1.1$.

Tabela 6.12: Sumário à Posteriori considerando $\alpha_{i}=\alpha$

\begin{tabular}{crcccc}
\hline Parâmetro & Média & DP & IC $95 \%$ & $\sqrt{\widehat{R}}$ & TAc \\
\hline \hline$\alpha$ & 0.9917 & 0.1692 & $(0.6545 ; 1.3228)$ & 1.0097 & 0.3354 \\
$\beta_{11}$ & 0.7552 & 0.2334 & $(0.3195 ; 1.2041)$ & 1.0003 & 0.7077 \\
$\beta_{12}$ & 0.5473 & 0.2323 & $(0.1149 ; 1.0113)$ & 1.0005 & 0.7452 \\
$\beta_{13}$ & -0.4081 & 0.2309 & $(-0.8732 ; 0.0384)$ & 1.0022 & 0.7328 \\
$\beta_{14}$ & 0.0254 & 0.2278 & $(-0.4064 ; 0.4601)$ & 1.0008 & 0.7216 \\
$\beta_{15}$ & 0.8289 & 0.2355 & $(0.3816 ; 1.2899)$ & 1.0005 & 0.7340 \\
$\beta_{16}$ & 0.2336 & 0.2456 & $(-0.2409 ; 0.7393)$ & 1.0010 & 0.7620 \\
$\beta_{21}$ & -1.1850 & 0.1654 & $(-1.5141 ;-0.8638)$ & 0.9993 & 0.4755 \\
$\beta_{22}$ & -0.7127 & 0.1540 & $(-1.0209 ;-0.3894)$ & 1.0062 & 0.4262 \\
$\beta_{23}$ & -0.6081 & 0.1519 & $(-0.9072 ;-0.3173)$ & 1.0026 & 0.4423 \\
$\beta_{24}$ & -0.6372 & 0.1521 & $(-0.9418 ;-0.3360)$ & 1.0029 & 0.4210 \\
$\beta_{25}$ & -0.8020 & 0.1626 & $(-1.1261 ;-0.4766)$ & 1.0050 & 0.4397 \\
$\beta_{26}$ & -0.3773 & 0.1686 & $(-0.6914 ;-0.0038)$ & 1.0025 & 0.4740 \\
$\beta_{31}$ & 0.1972 & 0.0610 & $(0.0823 ; 0.3176)$ & 1.0028 & 0.2327 \\
$\beta_{32}$ & 0.1097 & 0.0535 & $(0.0054 ; 0.2148)$ & 1.0014 & 0.1951 \\
$\beta_{33}$ & 0.0463 & 0.0548 & $(-0.0565 ; 0.1503)$ & 1.0002 & 0.1961 \\
$\beta_{34}$ & 0.0904 & 0.0496 & $(-0.0048 ; 0.1927)$ & 1.0050 & 0.1924 \\
$\beta_{35}$ & 0.1933 & 0.0591 & $(0.0794 ; 0.3144)$ & 1.0036 & 0.2031 \\
$\beta_{36}$ & 0.0746 & 0.0602 & $(-0.0386 ; 0.2024)$ & 1.0120 & 0.2261 \\
\hline \hline
\end{tabular}

A seguir apresentamos (tabela 6.13 e na figura 6.10) os resíduos de Pearson $d_{i j}$ (5.2), que expressa a medida de discrepância entre os dados e o modelo, dado por,

$$
d_{i j}=\frac{y_{i j}-\widetilde{p}_{i j}}{\sqrt{\widetilde{p}_{i j} \widetilde{q}_{i j}}}
$$

onde $\widetilde{p}_{i j}$ é $\theta_{i j}$ dado em (6.6) com os parâmetros estimados por Bayes (tabela 6.12) e $\widetilde{q}_{i j}=1-\widetilde{p}_{i j}, i=1,2, \ldots, 36 ; j=1,2, \ldots, 6$. 
Tabela 6.13: Resíduos de Pearson considerando $\alpha_{i}=\alpha$

\begin{tabular}{rrrrrr}
\hline & & \multicolumn{3}{c}{$\mathrm{J}$} & \\
1 & 2 & 3 & 4 & 5 & \multicolumn{1}{c}{6} \\
\hline \hline 0.8748 & 0.6112 & 1.0980 & 0.8529 & 0.5984 & 0.5656 \\
0.9969 & -1.3665 & -1.4133 & 0.6989 & 0.7352 & 0.5703 \\
0.5371 & 0.4600 & -1.0175 & -1.4609 & 0.3565 & 0.4655 \\
0.5371 & 0.4600 & -1.0175 & 0.6845 & 0.3565 & 0.4655 \\
0.7462 & 0.5570 & 1.0581 & 0.7930 & 0.5072 & 0.5305 \\
-1.3401 & 0.5570 & 1.0581 & -1.2610 & 0.5072 & -1.8849 \\
-0.1586 & 1.7825 & -0.9739 & 1.4475 & -0.2539 & 1.0465 \\
-0.3024 & 1.4825 & -0.6383 & -0.7385 & -0.8157 & 0.7817 \\
-1.1432 & 0.6112 & -0.9107 & -1.1725 & 0.5984 & 0.5656 \\
-0.1995 & -0.6154 & 0.9895 & -0.7438 & -0.3095 & -1.0141 \\
1.4107 & 0.8738 & -0.4680 & 1.2330 & 0.5331 & 0.6067 \\
1.0240 & 0.6695 & 1.1394 & 0.9169 & 0.7025 & -1.6601 \\
1.4086 & 0.8365 & 1.6082 & -0.8752 & -1.3903 & 0.6434 \\
0.6346 & 0.5067 & 1.0197 & 0.7370 & 0.4270 & 0.4972 \\
0.7462 & 0.5570 & -0.9451 & 0.7930 & 0.5072 & 0.5305 \\
2.2732 & 1.2401 & 1.4512 & -0.8545 & 0.8999 & 0.6923 \\
0.6346 & 0.5067 & 1.0197 & 0.7370 & 0.4270 & 0.4972 \\
1.0240 & -1.4936 & 1.1394 & 0.9169 & 0.7025 & -1.6601 \\
2.2658 & -0.8802 & -1.1719 & 1.0057 & -0.6233 & -1.2882 \\
-0.5237 & 1.1355 & -0.7157 & -0.9186 & 0.7704 & -1.5362 \\
-0.2476 & 1.4834 & 0.9536 & -0.8002 & -0.3733 & 0.9290 \\
-0.6038 & 0.8743 & -0.7849 & -0.8783 & -0.8934 & 0.7248 \\
-0.5086 & 1.0412 & -0.4306 & 1.4279 & -1.3581 & 0.6867 \\
-0.4399 & -0.8064 & 1.4512 & 1.1703 & 0.8999 & 0.6923 \\
-0.4413 & -0.8802 & 0.8534 & -0.9944 & 1.6044 & 0.7763 \\
-0.8568 & -1.2503 & 0.7348 & 0.7524 & 0.8593 & 0.6074 \\
-0.2467 & -0.6158 & 1.6282 & -0.6859 & -0.6973 & -1.2047 \\
-0.3024 & -0.6745 & -0.6383 & 1.3541 & -0.8157 & -1.2793 \\
-0.8328 & -1.3061 & -0.6462 & 1.0630 & 0.6131 & 0.6046 \\
-0.5253 & -0.9608 & -1.2163 & 0.9357 & 1.3682 & -1.3686 \\
-1.5758 & -1.9734 & -0.9806 & -1.3568 & 0.4270 & -2.0112 \\
-0.5253 & -0.9608 & -1.2163 & -1.0687 & 1.3682 & -1.3686 \\
-1.1399 & -1.4944 & -0.5286 & -1.0076 & 0.3124 & -1.9961 \\
-0.7109 & -1.2491 & -0.8148 & -0.9441 & -1.0425 & 0.6819 \\
-0.8328 & -1.3061 & -0.6462 & -0.9408 & -1.6311 & 0.6046 \\
\hline \hline & -0.6158 & -0.6142 & -0.6859 & -0.6973 & 0.8301 \\
\hline
\end{tabular}




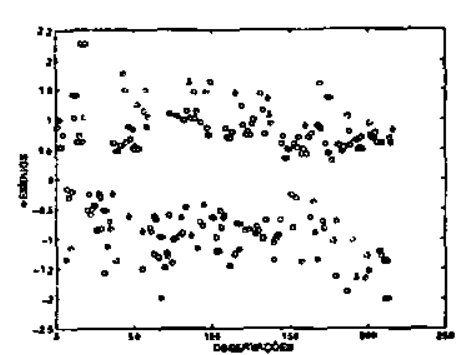

$$
j=1, \ldots, 6
$$

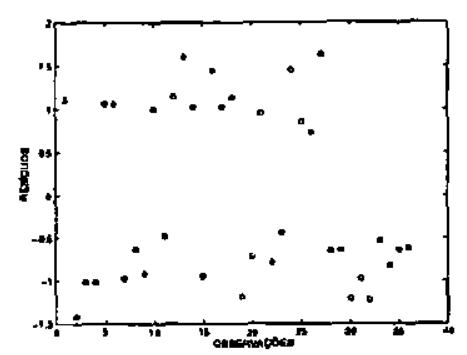

$$
j=3
$$

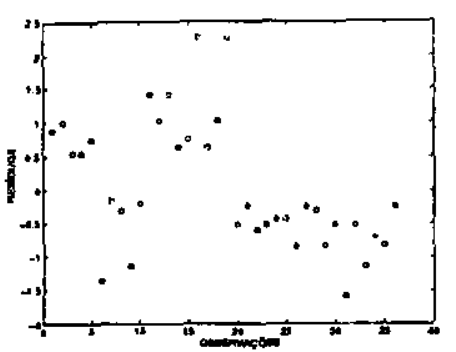

$j=1$

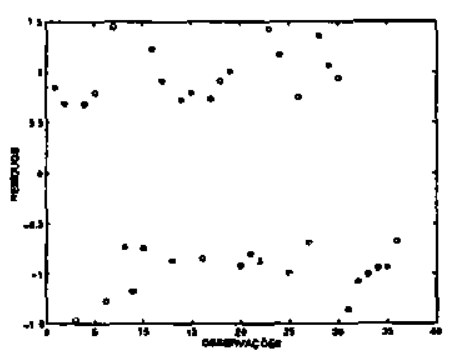

$j=4$

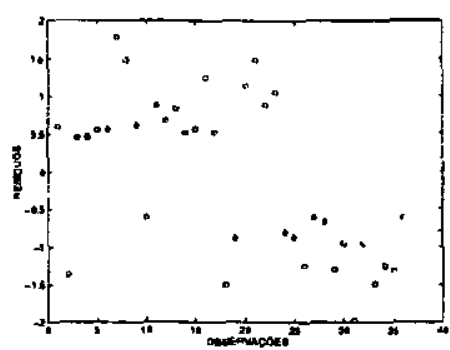

$j=2$

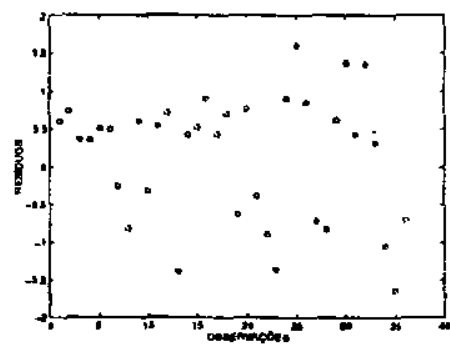

$j=5$

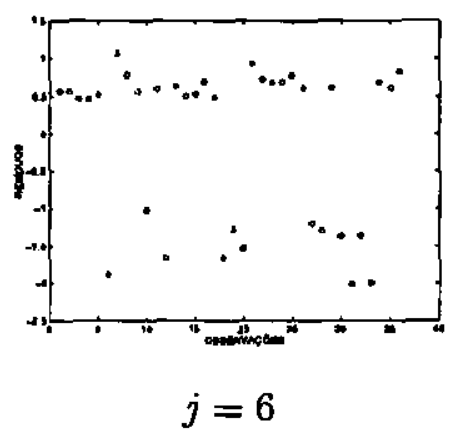

Figura 6.10: Resíduos de Pearson considerando $\alpha_{i}=\alpha, i=1,2, \ldots, 36$

\subsubsection{Aplicação do Modelo 5}

Para as densidades a priori (3.6) escolhemos os seguintes valores: $a=3, b=10 \mathrm{e}$ os $b_{k j}$ e $c_{k j}, k=1,2,3 ; j=1, \ldots, 6$ como sendo os mesmos usados no modelo 4 .

Na tabela 6.14 apresentamos o sumário a posteriori para os parâmetros do modelo 5 , a partir das amostras geradas das distribuições condicionais a posteriori obtidas de (3.7). Para obtermos este sumário, geramos 5 cadeias de 6000 iterações e consideramos uma amostra final de 1500 pontos amostrais, que foi suficiente para verificar a convergência observando que para todos os parâmetros $\sqrt{\widehat{R}}<1.1$. 
Tabela 6.14: Sumário à Posteriori considerando $\alpha_{i} \sim N\left(0, \sigma_{\alpha}^{2}\right)$

\begin{tabular}{crcccc}
\hline Parâmetro & Média & DP & IC $95 \%$ & $\sqrt{\widehat{R}}$ & TAc \\
\hline \hline$\sigma_{\alpha}^{2}$ & 0.1860 & 0.1526 & $(0.0240 ; 0.5871)$ & 1.0204 & - \\
$\beta_{11}$ & 1.1736 & 0.2356 & $(0.7242 ; 1.6318)$ & 1.0071 & 0.7227 \\
$\beta_{12}$ & 1.0833 & 0.2413 & $(0.6179 ; 1.5696)$ & 1.0010 & 0.7543 \\
$\beta_{13}$ & 0.1336 & 0.2310 & $(-0.3281 ; 0.5621)$ & 1.0046 & 0.7045 \\
$\beta_{14}$ & 0.5907 & 0.2326 & $(0.1386 ; 1.0485)$ & 1.0030 & 0.7223 \\
$\beta_{15}$ & 1.5074 & 0.2593 & $(1.0005 ; 2.0310)$ & 0.9991 & 0.7614 \\
$\beta_{16}$ & 0.6974 & 0.2482 & $(0.1914 ; 1.1748)$ & 1.0148 & 0.7656 \\
$\beta_{21}$ & -0.7696 & 0.1645 & $(-1.0881 ;-0.4615)$ & 1.0042 & 0.4806 \\
$\beta_{22}$ & -0.3106 & 0.1430 & $(-0.5850 ;-0.0285)$ & 1.0033 & 0.4560 \\
$\beta_{23}$ & -0.2505 & 0.1384 & $(-0.5343 ; 0.0196)$ & 0.9995 & 0.3329 \\
$\beta_{24}$ & -0.2780 & 0.1379 & $(-0.5403 ;-0.0118)$ & 1.0085 & 0.3848 \\
$\beta_{25}$ & -0.3539 & 0.1589 & $(-0.6742 ;-0.0523)$ & 1.0006 & 0.5274 \\
$\beta_{26}$ & 0.0688 & 0.1441 & $(-0.2118 ; 0.3603)$ & 1.0163 & 0.4409 \\
$\beta_{31}$ & 0.1317 & 0.0668 & $(0.0021 ; 0.2708)$ & 1.0044 & 0.2352 \\
$\beta_{32}$ & 0.0485 & 0.0565 & $(-0.0559 ; 0.1569)$ & 1.0085 & 0.1979 \\
$\beta_{33}$ & -0.0093 & 0.0545 & $(-0.1201 ; 0.0967)$ & 1.0024 & 0.1997 \\
$\beta_{34}$ & 0.0342 & 0.0542 & $(-0.0712 ; 0.1430)$ & 0.9994 & 0.1934 \\
$\beta_{35}$ & 0.1321 & 0.0612 & $(0.0206 ; 0.2581)$ & 1.0038 & 0.2240 \\
$\beta_{36}$ & -0.0082 & 0.0618 & $(-0.1272 ; 0.1121)$ & 1.0032 & 0.2172 \\
\hline \hline
\end{tabular}

A partir do modelo ajustado pelos parâmetros dados na tabela 6.14 calculamos os resíduos de Pearson $d_{i j}(5.2)$ dados na tabela 6.15. 
Tabela 6.15: Resíduos de Pearson $\left(\alpha_{i} \sim N\left(0, \sigma_{\alpha}^{2}\right)\right)$

\begin{tabular}{|c|c|c|c|c|c|}
\hline \\
\hline 1 & 2 & 3 & 4 & 5 & 6 \\
\hline 0.6658 & 0.4191 & 0.8089 & 0.6108 & 0.3428 & 0.3795 \\
\hline 1.3608 & -0.9939 & -0.9957 & 0.9916 & 0.9743 & 0.7769 \\
\hline 0.6675 & 0.5247 & -0.8792 & -1.2841 & 0.3435 & 0.5540 \\
\hline 0.5704 & 0.4445 & -1.0217 & 0.6680 & 0.2850 & 0.4703 \\
\hline 0.6057 & 0.4075 & 0.8268 & 0.6026 & 0.3065 & 0.3890 \\
\hline-1.0948 & 0.6328 & 1.2378 & -1.1002 & 0.4909 & -1.6480 \\
\hline-0.2141 & 1.4972 & -1.0661 & 1.3122 & -0.3287 & 0.7312 \\
\hline-0.2694 & 1.6483 & -0.5265 & -0.6128 & -0.8227 & 0.8644 \\
\hline-0.9908 & 0.6551 & -0.8184 & -1.0763 & 0.5474 & 0.5992 \\
\hline-0.1573 & -0.4963 & 1.3003 & -0.5635 & -0.2507 & -0.9865 \\
\hline 0.9548 & 0.5356 & -0.7182 & 0.8073 & 0.2302 & 0.3897 \\
\hline 0.8094 & 0.4824 & 0.8756 & 0.6873 & 0.4294 & -2.4016 \\
\hline 1.0854 & 0.5935 & 1.2170 & -1.1734 & -2.4390 & 0.4510 \\
\hline 0.5456 & 0.3927 & 0.8388 & 0.5897 & 0.2703 & 0.3951 \\
\hline 0.6928 & 0.4718 & -1.0623 & 0.6893 & 0.3590 & 0.4511 \\
\hline 1.8086 & 1.0112 & 1.2676 & -0.9760 & & \\
\hline 0.5445 & 0.3918 & 0.8371 & 0.5885 & 0.2696 & 0.3942 \\
\hline 0.9094 & -1.8273 & 0.9833 & 0.7736 & 0.4889 & -2.1072 \\
\hline 2.9106 & -0.6911 & -0.8749 & 1.3446 & -0.5023 & -1.1251 \\
\hline-0.3794 & 1.5197 & -0.4928 & -0.6414 & 0.9201 & -1.0915 \\
\hline-0.2787 & 1.4009 & 0.9643 & -0.7950 & -0.4158 & 0.7508 \\
\hline-0.5988 & 0.8275 & -0.7825 & -0.8960 & -1.0969 & 0.6301 \\
\hline-0.6749 & 0.7351 & -0.5770 & 1.0686 & -2.5133 & 0.4955 \\
\hline-0.4620 & -0.8389 & 1.4993 & 1.2081 & 0.7742 & 0.6876 \\
\hline-0.3966 & -0.7819 & 1.0126 & -0.8406 & 1.7471 & 0.7869 \\
\hline-0.6694 & -0.9697 & 0.9829 & 1.0047 & 1.0675 & 0.7608 \\
\hline-0.1975 & -0.5099 & 2.1581 & -0.5217 & -0.6511 & -1.0288 \\
\hline-0.2218 & -0.5195 & -0.4485 & 1.9052 & -0.7098 & -1.0002 \\
\hline-0.8354 & -1.4208 & -0.6632 & 1.0158 & 0.4579 & 0.5670 \\
\hline-0.3736 & -0.6938 & -0.8383 & 1.3552 & 1.8443 & -1.0792 \\
\hline-0.9248 & -1.2420 & -0.6051 & -0.8596 & 0.5887 & -1.2355 \\
\hline-0.3172 & -0.6028 & -0.7311 & -0.6420 & 2.1384 & -0.9427 \\
\hline-0.7834 & -1.1274 & -0.3615 & -0.7324 & 0.3337 & -1.3086 \\
\hline-0.5855 & -1.1065 & -0.6826 & -0.8110 & -1.0741 & 0.7228 \\
\hline-0.6435 & -1.0972 & -0.5056 & -0.7614 & -1.6555 & 0.7396 \\
\hline-0.1939 & -0.5029 & -0.4568 & -0.5145 & -0.6425 & 0.9845 \\
\hline
\end{tabular}

Na figura 6.11 apresentamos os resíduos de Pearson dados na tabela 6.15. 


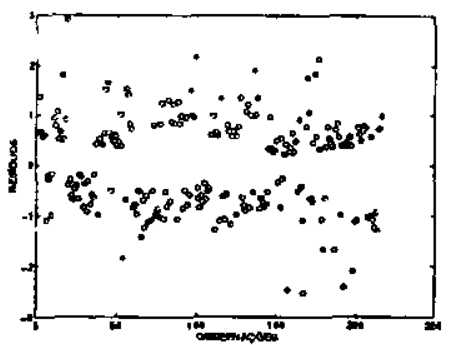

$j=1, \ldots, 6$

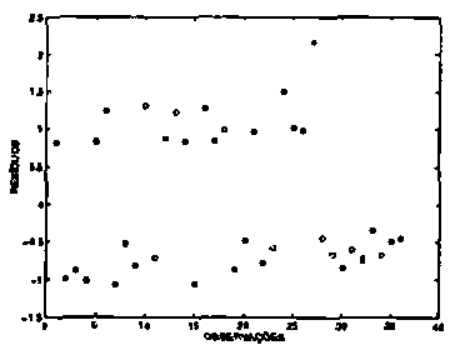

$j=3$

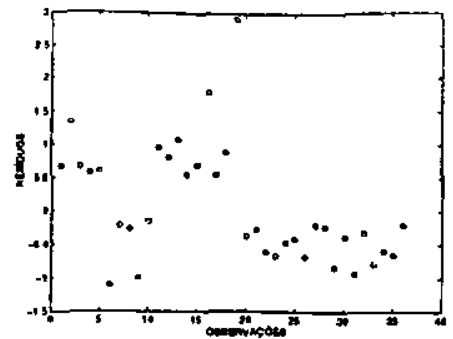

$j=1$

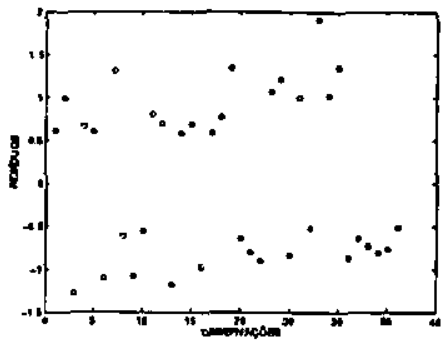

$j=4$

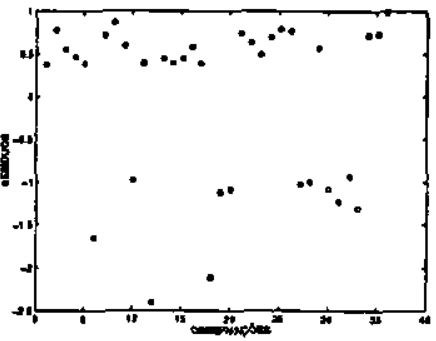

$j=6$

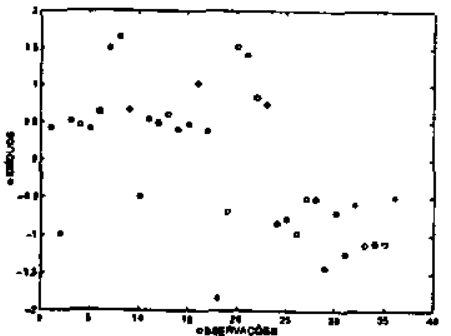

$j=2$

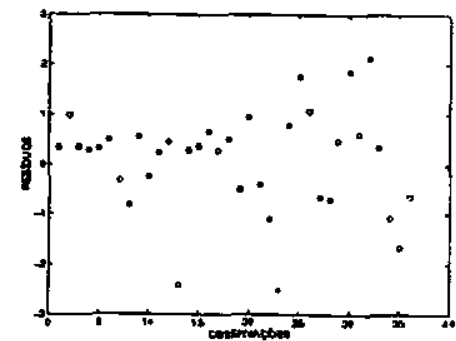

$j=5$

Figura 6.11: Resíduos de Pearson considerando $\alpha_{i}=\alpha, i=1,2, \ldots, 36$

\subsubsection{Aplicação do Modelo 6}

Para análise Bayesiana do modelo probito assumindo uma mistura de distribuições normais para os efeitos aleatórios $\alpha_{i}, i=1,2, \ldots, 36$, consideramos os seguintes valores para as densidades a priori (3.9): $d_{1}=-0.1, d_{2}=0.3, e_{1}=e_{1}=0.2, a_{1}=a_{2}=4$, $b_{1}=b_{2}=3, f=1, g=1$ e os valores para $b_{l j}$ e $c_{l j}, l=1,2,3 ; j=1,2, \ldots, 6$ os mesmos usados nos modelos 4 e 5 .

Os resumos a posteriori são apresentados na tabela 6.16, a seguir. 
Tabela 6.16: Sumário à Posteriori considerando $\alpha_{i}$ Mistura de Normais

\begin{tabular}{cccccc}
\hline Parâmetro & Média & DP & IC 95\% & $\sqrt{\widehat{R}}$ & TAc \\
\hline$\mu_{1}$ & -0.1307 & 0.4743 & $(-0.9566 ; 0.8289)$ & 1.0090 & - \\
$\mu_{2}$ & 0.4897 & 0.4070 & $(-0.5162 ; 1.1677)$ & 1.0115 & - \\
$\sigma_{1}^{2}$ & 0.1385 & 0.1051 & $(0.0427 ; 0.4204)$ & 1.0337 & - \\
$\sigma_{2}^{2}$ & 0.1308 & 0.0987 & $(0.0381 ; 0.3955)$ & 1.0088 & - \\
$p_{1}$ & 0.4337 & 0.2342 & $(0.0500 ; 0.9203)$ & 1.0252 & - \\
$\beta_{11}$ & 1.1476 & 0.2547 & $(0.6207 ; 1.6483)$ & 1.0032 & 0.7317 \\
$\beta_{12}$ & 1.0615 & 0.2451 & $(0.6107 ; 1.5566)$ & 1.0025 & 0.7583 \\
$\beta_{13}$ & 0.1067 & 0.2412 & $(-0.3927 ; 0.5662)$ & 1.0046 & 0.6738 \\
$\beta_{14}$ & 0.5789 & 0.2370 & $(0.1395 ; 1.0646)$ & 1.0002 & 0.7036 \\
$\beta_{15}$ & 1.5021 & 0.2772 & $(0.9593 ; 2.0489)$ & 1.0005 & 0.7662 \\
$\beta_{16}$ & 0.6976 & 0.2620 & $(0.2127 ; 1.2363)$ & 1.0021 & 0.7723 \\
$\beta_{21}$ & -0.8815 & 0.1769 & $(-1.2300 ;-0.5425)$ & 1.0093 & 0.4776 \\
$\beta_{22}$ & -0.4126 & 0.1669 & $(-0.7349 ;-0.0875)$ & 1.0020 & 0.4378 \\
$\beta_{23}$ & -0.3726 & 0.1617 & $(-0.6837 ;-0.0561)$ & 1.0037 & 0.4233 \\
$\beta_{24}$ & -0.4006 & 0.1607 & $(-0.7106 ;-0.0956)$ & 1.0056 & 0.4460 \\
$\beta_{25}$ & -0.4473 & 0.1840 & $(-0.7950 ;-0.0842)$ & 1.0017 & 0.4917 \\
$\beta_{26}$ & -0.0157 & 0.1852 & $(-0.3690 ; 0.3860)$ & 1.0034 & 0.4758 \\
$\beta_{31}$ & 0.0930 & 0.0676 & $(0.0164 ; 0.2802)$ & 1.0006 & 0.2359 \\
$\beta_{32}$ & 0.0574 & 0.0578 & $(-0.0631 ; 0.1721)$ & 1.0004 & 0.1951 \\
$\beta_{33}$ & 0.0040 & 0.0614 & $(-0.1222 ; 0.1206)$ & 1.0039 & 0.2030 \\
$\beta_{34}$ & 0.0552 & 0.0547 & $(-0.0461 ; 0.1647)$ & 1.0013 & 0.1989 \\
$\beta_{35}$ & 0.1493 & 0.0659 & $(0.0265 ; 0.2860)$ & 1.0017 & 0.2288 \\
$\beta_{36}$ & 0.0013 & 0.0719 & $(-0.1481 ; 0.1374)$ & 1.0030 & 0.2312 \\
\hline \hline
\end{tabular}

Na tabela 6.16 podemos observar pelo fator de redução de escala de $\sqrt{\widehat{R}}$ que houve a convergência para todos os parâmetros do modelo. Para conseguirmos a convergência dos parâmetros geramos 5 cadeias de 6000 iterações e desprezamos as 3000 primeiras iteraçōes considerando as amostras $3010^{\underline{a}}, 3020^{\underline{a}}, \ldots$

Abaixo apresentamos os resíduos de Pearson referentes ao modelo 6 para cada observação. 
Tabela 6.17: Resíduos de Pearson considerando $\alpha_{i}$ com Mistura de Normais

\begin{tabular}{rrrrrr}
\hline & \multicolumn{5}{c}{$\mathrm{J}$} \\
\multicolumn{1}{c}{2} & \multicolumn{1}{c}{3} & \multicolumn{1}{c}{4} & \multicolumn{1}{c}{5} & \multicolumn{1}{c}{6} \\
\hline 0.5054 & 0.3327 & 0.6817 & 0.5108 & 0.2660 & 0.2879 \\
1.1820 & -1.1963 & -1.1835 & 0.8294 & 0.7983 & 0.6330 \\
0.6667 & 0.4965 & -0.9097 & -1.3534 & 0.3124 & 0.5066 \\
0.5067 & 0.3698 & -1.1784 & 0.5646 & 0.2229 & 0.3779 \\
0.4687 & 0.3168 & 0.6836 & 0.4899 & 0.2288 & 0.2897 \\
-1.1326 & 0.6281 & 1.2585 & -1.0882 & 0.4799 & -1.7159 \\
-0.4064 & 1.2545 & -1.2092 & 1.2227 & -0.3799 & 0.6052 \\
-0.2047 & 1.5669 & -0.5388 & -0.6504 & -0.9175 & 0.7870 \\
-1.0463 & 0.6621 & -0.7881 & -1.0361 & 0.5494 & 0.5865 \\
-0.2493 & -0.5124 & 1.3202 & -0.5257 & -0.2451 & -1.0260 \\
1.1412 & 0.4724 & -0.7848 & 0.6994 & 0.1748 & 0.3147 \\
0.5876 & 0.3805 & 0.7318 & 0.5750 & 0.3360 & -3.1919 \\
0.9978 & 0.5101 & 1.0913 & -1.3328 & -3.0010 & 0.3632 \\
0.4333 & 0.3008 & 0.6845 & 0.4688 & 0.1950 & 0.2910 \\
0.5702 & 0.3934 & -1.2177 & 0.5948 & 0.2900 & 0.3617 \\
2.2502 & 0.8889 & 1.1361 & -1.1417 & 0.5282 & 0.4794 \\
0.4420 & 0.3075 & 0.6969 & 0.4780 & 0.1999 & 0.2975 \\
0.7017 & -2.1618 & 0.8694 & 0.6871 & 0.4111 & -2.5981 \\
2.2095 & -0.7596 & -0.9313 & 1.2945 & -0.5436 & -1.2498 \\
-0.2486 & 1.4773 & -0.4987 & -0.6860 & 0.8259 & -1.1751 \\
-0.4550 & 1.2021 & 0.8635 & -0.8504 & -0.4716 & 0.6359 \\
-0.7048 & 0.8789 & -0.7059 & -0.7890 & -1.0129 & 0.6510 \\
-0.5752 & 0.6882 & -0.5954 & 0.9951 & -2.9372 & 0.4303 \\
-0.3718 & -0.9640 & 1.3277 & 1.0223 & 0.6224 & 0.5670 \\
-0.5443 & -0.8997 & 0.9078 & -0.9148 & 1.5406 & 0.6741 \\
-0.8103 & -1.1791 & 0.8213 & 0.8400 & 0.8720 & 0.6132 \\
-0.1293 & -0.4729 & 2.4142 & -0.4835 & -0.6412 & -1.0043 \\
-0.1432 & -0.4925 & -0.4114 & 1.9902 & -0.7183 & -0.9963 \\
-0.7666 & -1.4357 & -0.6503 & 1.0112 & 0.4326 & 0.5336 \\
-0.4653 & -0.7568 & -0.8891 & 1.3017 & 1.7078 & -1.1902 \\
-0.7761 & -1.0546 & -0.4971 & -0.7227 & 0.6833 & -1.0828 \\
-0.3519 & -0.5895 & -0.6962 & -0.5988 & 2.2218 & -0.9346 \\
-0.4054 & -0.9155 & -0.2733 & -0.6255 & 0.3799 & -1.1320 \\
-0.5895 & -0.9314 & -0.5474 & -0.6416 & -0.8923 & 0.8357 \\
-0.4793 & -0.9165 & -0.3996 & -0.6292 & -1.4318 & 0.8465 \\
-0.1237 & -0.4586 & -0.4012 & -0.4689 & -0.6230 & 1.0237 \\
\hline \hline & & & & & \\
\hline & & & -1.0
\end{tabular}

Na figura 6.12 temos os resíduos de Pearson referentes ao modelo 6 para melhor 
visualização.

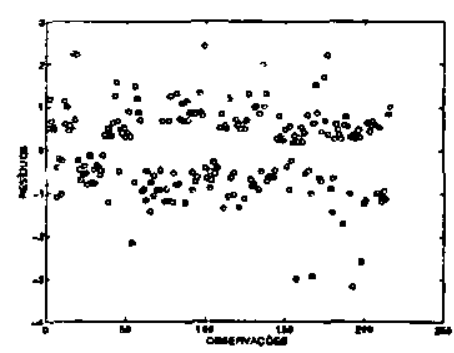

$j=1, \ldots, 6$

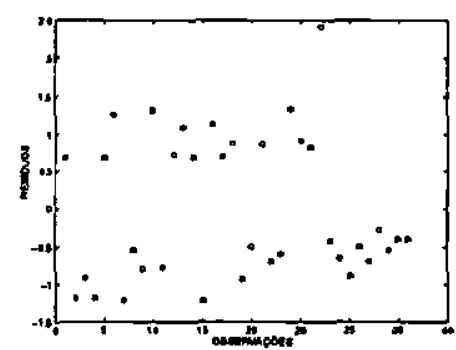

$j=3$

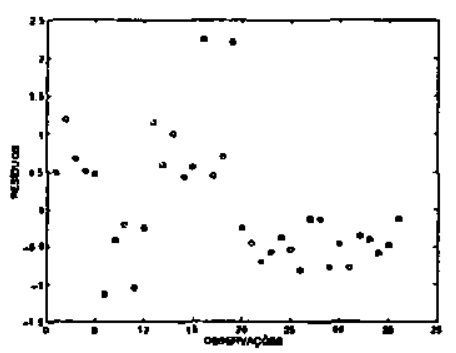

$j=1$

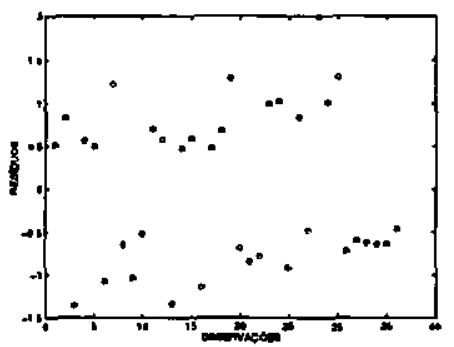

$j=4$

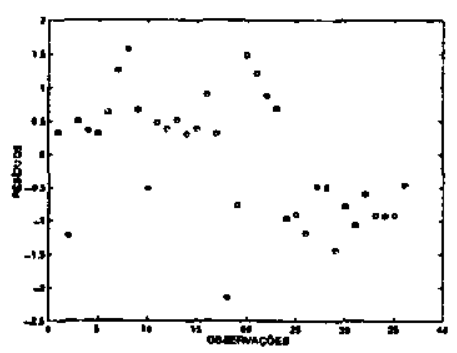

$j=2$

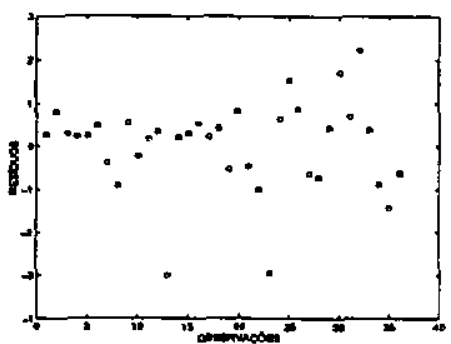

$j=5$

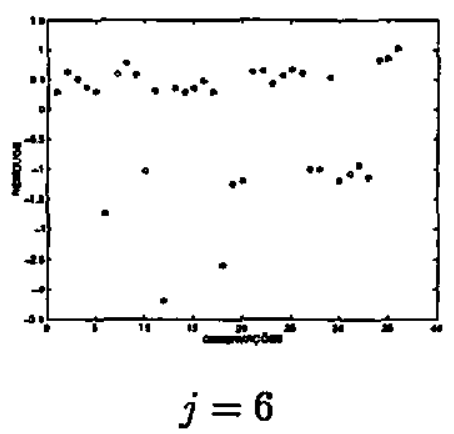

Figura 6.12: Resíduos de Pearson considerando $\alpha_{i}=\alpha, i=1,2, \ldots, 36$

\subsubsection{Comparação dos Modelos 4, 5 e 6}

Para observar o comportamento dos parâmetros sob os diferentes modelos produzimos a tabela 6.18 que contém um resumo dos estimadores de MCMC para a média a posteriori dos parâmetros $\beta_{l j}(l=1,2,3 ; j=1,2, \ldots, 6)$ dos modelos 4,5 e 6 que consideram a função de ligação probito generalizada. 
Tabela 6.18: Sumário à Posteriori para $\beta_{j}$

\begin{tabular}{|c|c|c|c|c|}
\hline Parâmetro & EMV & $\alpha$ fixo & $\alpha_{i} \sim N\left(0, \sigma_{\alpha}^{2}\right)$ & $\alpha_{i} \sim$ Mistura de Normais \\
\hline$\beta_{11}$ & 0.7799 & 0.7328 & 1.1736 & 1.1476 \\
\hline$\beta_{12}$ & 0.5650 & 0.5383 & 1.0833 & 1.0615 \\
\hline$\beta_{13}$ & -0.4330 & -0.4261 & 0.1336 & 0.1067 \\
\hline$\beta_{14}$ & 0.0336 & 0.0426 & 0.5907 & 0.5789 \\
\hline$\beta_{15}$ & 0.8677 & 0.8219 & 1.5074 & 1.5021 \\
\hline$\beta_{16}$ & 0.2403 & 0.2355 & 0.6974 & 0.6976 \\
\hline$\beta_{21}$ & -1.2116 & -1.1880 & -0.7696 & -0.8815 \\
\hline$\beta_{22}$ & -0.7623 & -0.7160 & -0.3106 & -0.4126 \\
\hline$\beta_{23}$ & -0.6351 & -0.6065 & -0.2505 & -0.3726 \\
\hline$\beta_{24}$ & -0.6830 & -0.6385 & -0.2780 & -0.4006 \\
\hline$\beta_{25}$ & -0.8444 & -0.8069 & -0.3539 & -0.4473 \\
\hline$\beta_{26}$ & -0.4452 & -0.3769 & 0.0688 & -0.0157 \\
\hline$\beta_{31}$ & 0.2012 & 0.1983 & 0.1317 & 0.0930 \\
\hline$\beta_{32}$ & 0.1129 & 0.1103 & 0.0485 & 0.0574 \\
\hline$\beta_{33}$ & 0.0549 & 0.0462 & -0.0093 & 0.0040 \\
\hline$\beta_{34}$ & 0.0973 & 0.0897 & 0.0342 & 0.0552 \\
\hline$\beta_{35}$ & 0.1968 & 0.1934 & 0.1321 & 0.1493 \\
\hline$\beta_{36}$ & 0.0698 & 0.0691 & -0.0082 & 0.0013 \\
\hline
\end{tabular}

Na tabela 6.19, apresentamos o Total de Pearson (5.3) e as densidades preditivas para verificação da performance dos modelos.

Tabela 6.19: Resultados do Total de Pearson (6.4) e as Densidades Preditivas para os Modelos

\begin{tabular}{c|ccc}
\hline & Modelo 4 & Modelo 5 & Modelo 6 \\
\hline \hline$D(36$ obs $)$ & 209.4069 & 192.7197 & 191.9622 \\
$\widehat{p}_{2}\left(\widehat{y} \mid M_{k}\right)(36 \mathrm{obs})$ & $1.2669 \cdot 10^{-67}$ & $4.3601 \cdot 10^{-64}$ & $3.3753 \cdot 10^{-62}$ \\
\hline \hline
\end{tabular}

Pelos valores de $D$ podemos constatar uma melhor performance do modelo de efeitos aleatórios com uma mistura de duas distribuiçōes normais, modelo 6 sobre os modelos 4 e 5 .

Calculando o fator de Bayes (5.11), temos,

- $\operatorname{modelos} 6$ e $4-B_{64}=2.6642 \cdot 10^{5}$

- modelos 6 e $5-B_{65}=77.4134$

- $\operatorname{modelos} 5$ e $4-B_{54}=3.4416 \cdot 10^{3}$ 
verificamos uma forte evidência a favor do modelo 6 sobre os modelos 4 e 5 (tabela 5.1).

A seguir temos nas figura 6.13 e 6.14, histogramas que mostram as frequências dos $\alpha_{i}, i=1,2, \ldots, 36$ para os modelos 5 e 6 respectivamente.

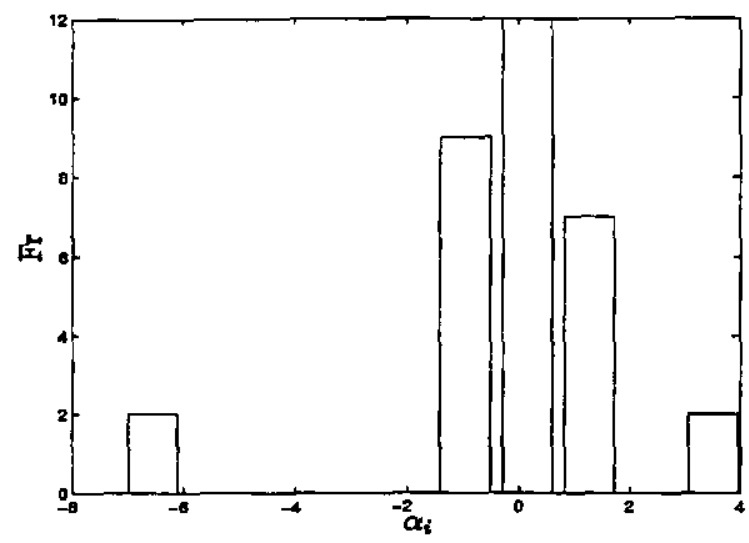

Figura 6.13: Frequências dos $\alpha_{i}$ Assumindo $\alpha_{i} \stackrel{i i d}{\sim} N\left(0, \sigma_{\alpha}^{2}\right) i=1,2, \ldots, 36$

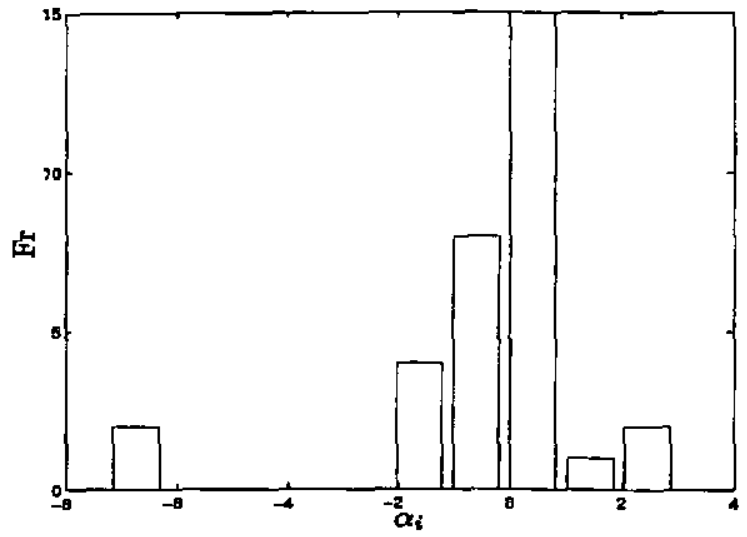

Figura 6.14: Frequências dos $\alpha_{i}$ Assumindo $\alpha_{i}, i=1,2, \ldots, 36$ Mistura de duas Normais

\subsection{Ilustração Numérica para o Capítulo 4}

Para ilustrar o capítulo 4 usamos o conjunto de dados (tabela 6.20) introduzido por Chib e Greenberg (1998), que contêm medidas binárias repetidas do estado de bronquite para 537 crianças em idades de 7,8,9 e 10 anos de Stuebenville, Ohio. O objetivo do estudo é modelar a probabilidade do estado de bronquite com o passar do tempo, em função de uma variável indicadora binária, que representa, mães que possuem o hábito de fumar durante o primeiro ano do estudo. 
Tabela 6.20: Frequência de Crianças com Bronquite

\begin{tabular}{|c|c|c|c|c|c|c|c|c|c|}
\hline \multicolumn{5}{|c|}{$\begin{array}{l}\text { Mãe fumante } \\
\text { Idade da criança }\end{array}$} & \multicolumn{5}{|c|}{$\begin{array}{l}\text { Mãe não fumante } \\
\text { Idade da criança }\end{array}$} \\
\hline 7 & 8 & 9 & & Frequência & 7 & 8 & 9 & 10 & Frequência \\
\hline 0 & 0 & 0 & 0 & 237 & 0 & 0 & 0 & 0 & 118 \\
\hline 0 & 0 & 0 & 1 & 10 & 0 & 0 & 0 & 1 & 6 \\
\hline 0 & 0 & 1 & 0 & 15 & 0 & 0 & 1 & 0 & 8 \\
\hline 0 & 0 & 1 & 1 & 4 & 0 & 0 & 1 & 1 & 2 \\
\hline 0 & 1 & 0 & 0 & 16 & 0 & 1 & 0 & 0 & 11 \\
\hline 0 & 1 & 0 & 1 & 2 & 0 & 1 & 0 & 1 & 1 \\
\hline 0 & 1 & 1 & 0 & 7 & 0 & 1 & 1 & 0 & 6 \\
\hline 0 & 1 & 1 & 1 & 3 & 0 & 1 & 1 & 1 & 4 \\
\hline 1 & 0 & 0 & 0 & 24 & 1 & 0 & 0 & 0 & 7 \\
\hline 1 & 0 & 0 & 1 & 3 & 1 & 0 & 0 & 1 & 3 \\
\hline 1 & 0 & 1 & 0 & 3 & 1 & 0 & 1 & 0 & 3 \\
\hline 1 & 0 & 1 & 1 & 2 & 1 & 0 & 1 & 1 & 1 \\
\hline 1 & 1 & 0 & 0 & 6 & 1 & 1 & 0 & 0 & 4 \\
\hline 1 & 1 & 0 & 1 & 2 & 1 & 1 & 0 & 1 & 2 \\
\hline 1 & 1 & 1 & 0 & 5 & 1 & 1 & 1 & 0 & 4 \\
\hline 1 & 1 & 1 & 1 & 11 & 1 & 1 & 1 & 1 & 7 \\
\hline
\end{tabular}

Temos então:

$N=537:$ número de crianças observadas;

$n=32$ : número de grupos de frequência $f_{i}, i=1,2, \ldots, 32$;

$J=4:$ número de tempos, $j=1,2,3,4$

$y_{i j}$ : a resposta para a $i$-ésima observação no $j$-ésimo tempo, onde:

$$
y_{i j}= \begin{cases}1 & \text { com bronquite } \\ 0 & \text { sem bronquite. }\end{cases}
$$

$x_{i j}=x_{i j 1}:$ covariada de regressão, onde:

$$
x_{i j}= \begin{cases}1 & \text { se a mãe é fumante, } \\ 0 & \text { se a mãe não é fumante. }\end{cases}
$$


Assim $Y_{i j}(0$ ou 1$)$, trata-se de resposta binária com distribuição de Bernoulli $\left(\theta_{i j}\right)$, ou seja,

$$
\begin{array}{r}
\operatorname{Pr}\left[Y_{i j}=y_{i j} \mid \alpha_{i}, \beta_{j}, x_{i j}\right]=\theta_{i j}^{y_{i j}}\left(1-\theta_{i j}\right)^{1-y_{i j}} \\
i=1,2, \ldots, 32 ; \quad j=1,2,3,4
\end{array}
$$

onde $\theta_{i j}$ tem duas possibilidades:

a) $\theta_{i j}=\frac{\exp \left(\alpha_{i}+x_{i j 1} \beta_{1 j}\right)}{1+\exp \left(\alpha_{i}+x_{i j 1} \beta_{1 j}\right)} \quad i=1,2, \ldots, 32 ; j=1,2,3,4$ (Logístico)

b) $\theta_{i j}=\Phi\left(\alpha_{i}+x_{i j 1} \beta_{1 j}\right) \quad i=1,2, \ldots, 32 ; j=1,2,3,4$ (Probito)

Para o modelo que usa a função de ligação logística, a função de verossimilhança é dada por,

$$
\left.\mathrm{L} \underset{\sim}{\alpha}, \beta_{1} \mid \mathcal{D}\right)=\prod_{i=1}^{32} \prod_{j=1}^{4}\left[\frac{\exp \left\{\left(\alpha_{i}+x_{i j 1} \beta_{1 j}\right) y_{i j}\right\}}{1+\exp \left(\alpha_{i}+x_{i j 1} \beta_{1 j}\right)}\right]^{f_{i}}
$$

onde $\underset{\sim}{\alpha}=\left(\alpha_{1}, \alpha_{2}, \ldots, \alpha_{32}\right)$ e $f_{i}, i=1,2, \ldots, 32$ a frequência de cada grupo.

A função de verossimilhança para o modelo de função de ligação probito é dada por,

$$
\mathrm{L}\left(\underset{\sim}{\alpha}, \beta_{1} \mid \mathcal{D}\right)=\prod_{i=1}^{32} \prod_{j=1}^{4}\left[\Phi\left(\alpha_{i}+x_{i j 1} \beta_{1 j}\right)^{y_{i j}}\left[1-\Phi\left(\alpha_{i}+x_{i j 1} \beta_{1 j}\right)\right]^{1-y_{i j}}\right]^{f_{i}}
$$

onde $\underset{\sim}{\alpha}=\left(\alpha_{1}, \alpha_{2}, \ldots, \alpha_{32}\right)$ e $f_{i}, i=1,2, \ldots, 32$ a frequência de cada grupo.

Para exemplificar o capítulo 4 faremos as seguintes considerações:

i) Para função de ligação logística:

Modelo 7: Modelo logístico de efeitos fixos;

Modelo 8: Modelo logístico de efeitos aleatórios considerando $\alpha_{i} \stackrel{i i d}{\sim} N\left(0, \sigma_{\alpha}^{2}\right)$,

$$
i=1,2, \ldots, 32 \text {; }
$$

Modelo 9: Modelo logístico de efeitos aleatórios com uma mistura de duas distribuiçōes Normais. 
i) Para função de ligação probito:

Modelo 10: Modelo probito de efeitos fixos;

Modelo 11: Modelo probito de efeitos aleatórios considerando $\alpha_{i} \stackrel{i i d}{\sim} N\left(0, \sigma_{\alpha}^{2}\right)$, $i=1,2, \ldots, 32$;

Modelo 12: Modelo probito de efeitos aleatórios com uma mistura de duas distribuiçōes Normais.

\subsubsection{EMV para os Parâmetros do Modelo com Ligação Logística Considerando Réplicas}

A tabela 6.21 apresenta os estimadores de máxima verossimilhança para os parâmetros sob o modelo 7 .

Tabela 6.21: Estimadores de máxima verosimilhança

\begin{tabular}{ccc}
\hline Parâmetro & EMV & Int. Conf. (95\%) \\
\hline \hline$\alpha$ & -1.8212 & $(-1.9393 ;-1.9393)$ \\
$\beta_{11}$ & 0.2054 & $(-0.1801 ;-0.1801)$ \\
$\beta_{12}$ & 0.4876 & $(0.1348 ; 0.1348)$ \\
$\beta_{13}$ & 0.3527 & $(-0.0148 ;-0.0148)$ \\
$\beta_{14}$ & -0.0021 & $(-0.4163 ;-0.4163)$ \\
\hline
\end{tabular}

\subsubsection{Aplicação do Modelo 7}

Considerando as distribuições a priori (4.7) com $\mu_{0}=-1.8, \sigma_{0}=0.3, b_{11}=0.2$, $b_{12}=0.4, b_{13}=0.3, b_{14}=0, c_{1 j}=0.3, j=1,2,3,4$, geramos 5 cadeias cada uma com 6000 iterações e tomamos as $3010^{a}, 3020^{\underline{a}}$, ... iterações de cada cadeia para formar a amostra. Os resumos a posteriori são apresentados na tabela 6.22.

Tabela 6.22: Sumários a posteriori

\begin{tabular}{cccccc}
\hline Parâmetro & Média & DP & IC $(95 \%)$ & $\sqrt{\widehat{R}}$ & TAc \\
\hline \hline$\alpha$ & -1.7928 & 0.2968 & $(-2.3935 ;-1.2271)$ & 1.0052 & 0.9998 \\
$\beta_{11}$ & 0.2022 & 0.3014 & $(-0.3978 ; 0.7859)$ & 1.0026 & 0.9997 \\
$\beta_{12}$ & 0.3990 & 0.3021 & $(-0.1904 ; 0.9741)$ & 0.9999 & 0.9989 \\
$\beta_{13}$ & 0.2953 & 0.3009 & $(-0.2579 ; 0.9181)$ & 0.9999 & 0.9996 \\
$\beta_{14}$ & -0.0043 & 0.2925 & $(-0.5554 ; 0.5906)$ & 1.0003 & 0.9998 \\
\hline \hline
\end{tabular}


Na tabela 6.23 apresentamos os resíduos de Pearson para o modelo 7.

Tabela 6.23: Resíduos de Pearson assumindo

\begin{tabular}{|c|c|c|c|}
\hline \multicolumn{4}{|c|}{$\alpha_{i}=\alpha(i=1,2, \ldots, 32)$} \\
\hline \multicolumn{4}{|c|}{$\mathbf{J}$} \\
\hline 1 & 2 & 3 & 4 \\
\hline-0.4080 & -0.4080 & -0.4080 & -0.4080 \\
\hline-0.4080 & -0.4080 & -0.4080 & 2.4508 \\
\hline-0.4080 & -0.4080 & 2.4508 & -0.4080 \\
\hline-0.4080 & -0.4080 & 2.4508 & 2.4508 \\
\hline-0.4080 & 2.4508 & -0.4080 & -0.4080 \\
\hline-0.4080 & 2.4508 & -0.4080 & 2.4508 \\
\hline-0.4080 & 2.4508 & 2.4508 & -0.4080 \\
\hline-0.4080 & 2.4508 & 2.4508 & 2.4508 \\
\hline 2.4508 & -0.4080 & -0.4080 & -0.4080 \\
\hline 2.4508 & -0.4080 & -0.4080 & 2.4508 \\
\hline 2.4508 & -0.4080 & 2.4508 & -0.4080 \\
\hline 2.4508 & -0.4080 & 2.4508 & 2.4508 \\
\hline 2.4508 & 2.4508 & -0.4080 & -0.4080 \\
\hline 2.4508 & 2.4508 & -0.4080 & 2.4508 \\
\hline 2.4508 & 2.4508 & 2.4508 & -0.4080 \\
\hline 2.4508 & 2.4508 & 2.4508 & 2.4508 \\
\hline-0.4514 & -0.4981 & -0.4730 & -0.4072 \\
\hline-0.4514 & -0.4981 & -0.4730 & 2.4560 \\
\hline-0.4514 & -0.4981 & 2.1144 & -0.4072 \\
\hline-0.4514 & -0.4981 & 2.1144 & 2.4560 \\
\hline-0.4514 & 2.0075 & -0.4730 & -0.4072 \\
\hline-0.4514 & 2.0075 & -0.4730 & 2.4560 \\
\hline-0.4514 & 2.0075 & 2.1144 & -0.4072 \\
\hline-0.4514 & 2.0075 & 2.1144 & 2.4560 \\
\hline 2.2151 & -0.4981 & -0.4730 & -0.4072 \\
\hline 2.2151 & -0.4981 & -0.4730 & 2.4560 \\
\hline 2.2151 & -0.4981 & 2.1144 & -0.4072 \\
\hline 2.2151 & -0.4981 & 2.1144 & 2.4560 \\
\hline 2.2151 & 2.0075 & -0.4730 & -0.4072 \\
\hline 2.2151 & 2.0075 & -0.4730 & 2.4560 \\
\hline 2.2151 & 2.0075 & 2.1144 & -0.4072 \\
\hline 2.2151 & 2.0075 & 2.1144 & 2.4560 \\
\hline
\end{tabular}

A seguir apresentamos os gráficos dos resíduos de Pearson $d_{i j}$. 

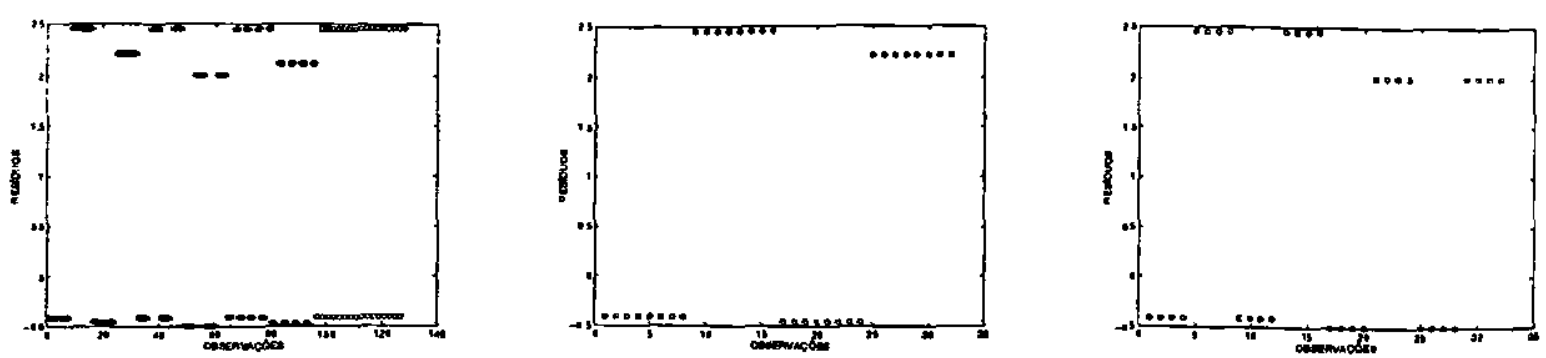

$j=1, \ldots, 4$

$j=1$

$$
j=2
$$

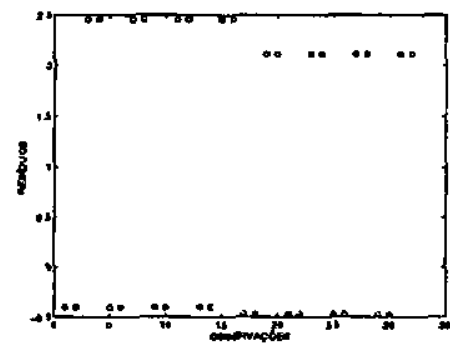

$j=3$

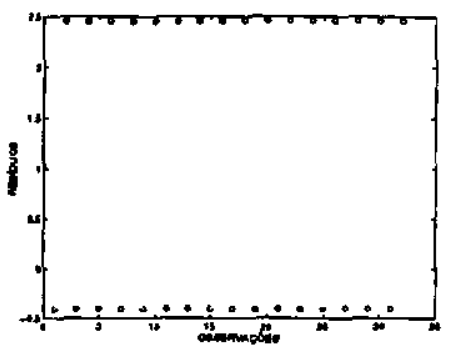

$j=4$

Figura 6.15: Resíduos de Pearson considerando $\alpha_{i}=\alpha(i=1,2, \ldots, 32)$

\subsubsection{Aplicação do Modelo 8}

Para o modelo 8, modelo logístico de efeitos aleatórios com distribuição normal, consideramos os seguintes valores para as distribuiçōes a priori (4.9): $a=3, b=8$, para $b_{1 j}$ e $c_{1 j}(j=1,2,3,4)$ os mesmos valores usados no modelo de efeitos fixos, modelo 7 . Para obtermos o sumário a posteriori (Tabela 6.24) geramos 5 cadeias de 6000 amostras e desprezamos as 3000 primeiras, e assim selecionamos 1500 pontos amostrais com saltos de 10 iterações, sendo suficiente para que $\sqrt{\widehat{R}}<1.1$ para todos os parâmetros do modelo.

Tabela 6.24: Sumário à Posteriori considerando $\alpha_{i} \sim N\left(0, \sigma_{\alpha}^{2}\right)$

\begin{tabular}{crcccc}
\hline Parâmetro & Média & DP & IC 95\% & $\sqrt{\widehat{R}}$ & TAc \\
\hline \hline$\sigma_{\alpha}^{2}$ & 4.6162 & 1.5449 & $(2.4822 ; 8.2327)$ & 1.0112 & - \\
$\beta_{11}$ & 0.1935 & 0.1976 & $(-0.1788 ; 0.5750)$ & 1.0038 & 0.9976 \\
$\beta_{12}$ & 0.4032 & 0.1986 & $(0.0128 ; 0.7987)$ & 1.0021 & 0.9526 \\
$\beta_{13}$ & 0.2940 & 0.2040 & $(-0.1144 ; 0.7125)$ & 0.9988 & 0.4333 \\
$\beta_{14}$ & -0.0058 & 0.2006 & $(-0.4119 ; 0.3814)$ & 1.0012 & 0.7542 \\
\hline
\end{tabular}

A seguir apresentamos os valores (tabela 6.25) e os gráficos (figura 6.16) do resíduo de Pearson para os dados. 
Tabela 6.25: Resíduos de Pearson $\left(\alpha_{i} \sim N\left(0, \sigma_{\alpha}^{2}\right)\right)$

\begin{tabular}{|c|c|c|c|}
\hline \multicolumn{4}{|c|}{$\mathrm{J}$} \\
\hline 1 & 2 & 3 & 4 \\
\hline-0.0286 & -0.0286 & -0.0286 & -0.0286 \\
\hline-0.5952 & -0.5952 & -0.5952 & 1.6801 \\
\hline-0.6128 & -0.6128 & 1.6317 & -0.6128 \\
\hline-0.9690 & -0.9690 & 1.0320 & 1.0320 \\
\hline-0.5931 & 1.6860 & -0.5931 & -0.5931 \\
\hline-0.9542 & 1.0480 & -0.9542 & 1.0480 \\
\hline-0.9123 & 1.0961 & 1.0961 & -0.9123 \\
\hline-1.6409 & 0.6094 & 0.6094 & 0.6094 \\
\hline 1.6746 & -0.5971 & -0.5971 & -0.5971 \\
\hline 1.0252 & -0.9754 & -0.9754 & 1.0252 \\
\hline 1.0346 & -0.9665 & 1.0346 & -0.9665 \\
\hline 0.6314 & -1.5838 & 0.6314 & 0.6314 \\
\hline 1.0255 & 1.0255 & -0.9751 & -0.9751 \\
\hline 0.6271 & 0.6271 & -1.5946 & 0.6271 \\
\hline 0.5940 & 0.5940 & 0.5940 & -1.6836 \\
\hline 0.1309 & 0.1309 & 0.1309 & 0.1309 \\
\hline-0.0445 & -0.0494 & -0.0468 & -0.0402 \\
\hline-0.5540 & -0.6153 & -0.5826 & 1.9941 \\
\hline-0.5878 & -0.6527 & 1.6180 & -0.5320 \\
\hline-0.9381 & -1.0419 & 1.0137 & 1.1776 \\
\hline-0.6707 & 1.3426 & -0.7053 & -0.6071 \\
\hline-0.9166 & 0.9824 & -0.9638 & 1.2053 \\
\hline-0.8979 & 1.0029 & 1.0592 & -0.8127 \\
\hline-1.6326 & 0.5515 & 0.5825 & 0.6767 \\
\hline 1.7896 & -0.6205 & -0.5876 & -0.5058 \\
\hline 1.0590 & -1.0487 & -0.9930 & 1.1699 \\
\hline 1.0459 & -1.0618 & 0.9946 & -0.8655 \\
\hline 0.6706 & -1.6562 & 0.6377 & 0.7408 \\
\hline 1.0462 & 0.9420 & -1.0051 & -0.8652 \\
\hline 0.6312 & 0.5683 & -1.6660 & 0.6973 \\
\hline 0.6112 & 0.5504 & 0.5812 & -1.4810 \\
\hline 0.1590 & 0.1432 & 0.1512 & 0.1757 \\
\hline
\end{tabular}

Os valores apresentados na tabela 6.25 são colocados em forma de gráfico na figura 6.16. 


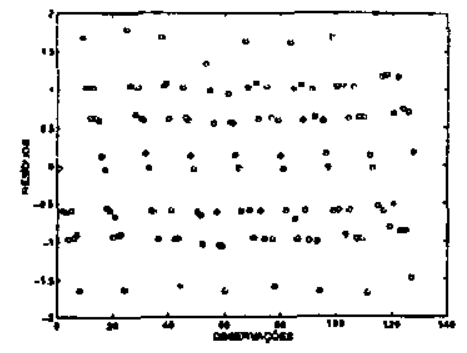

$j=1, \ldots, 4$

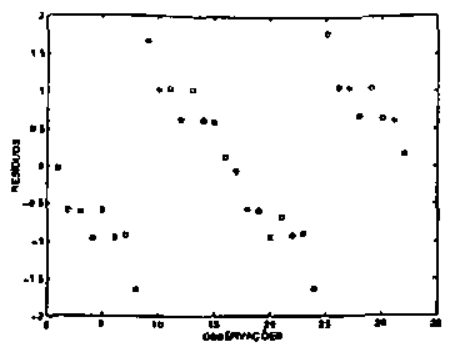

$j=1$

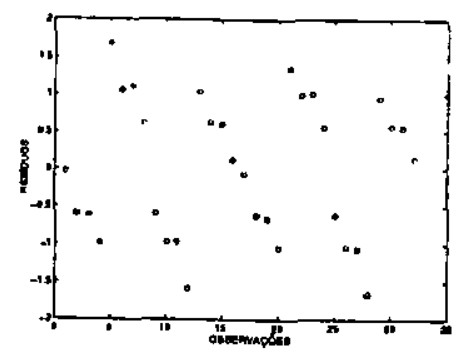

$j=2$

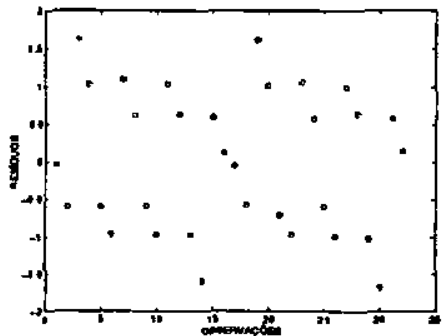

$j=3$

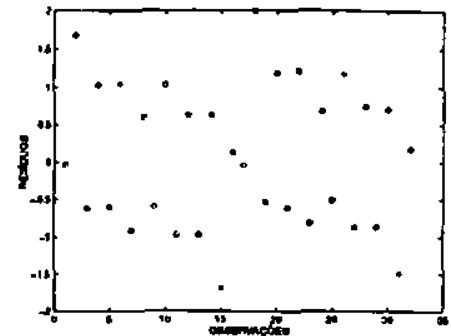

$j=4$

Figura 6.16: Resíduos de Pearson considerando $\alpha_{i} \sim N\left(0, \sigma_{\alpha}^{2}\right), i=1,2, \ldots, 32$

\subsubsection{Aplicação do Modelo 9}

Assumimos uma mistura de duas distribuições normais (4.10) para os efeitos aleatórios $\alpha_{i}, i=1,2, \ldots, 32$ e distribuições a priori (4.11) com $d_{1}=-6.7, e_{1}=0.4$, $d_{2}=0.5, e_{2}=1.2, a_{1}=8, b_{1}=4, a_{2}=3, b_{2}=0.3, f=1, g=1$ e os valores para $b_{l j}$ e $c_{l j}$ os mesmos usados para o modelo 7. Foram feitas 5 cadeias de 6000 iterações cada, dessas descartamos as 3000 primeiras, e assim foram tomadas amostras de 10 em 10, portanto as inferências foram feitas sobre amostras de 1500 observações.

Tabela 6.26: Sumário à Posteriori considerando $\alpha_{i}$ com Mistura de Normais

\begin{tabular}{cccccc}
\hline Parâmetro & Méd. & STD & IC 95\% & $\sqrt{\widehat{R}}$ & TAc \\
\hline \hline$\mu_{2}$ & -7.2261 & 0.5277 & $(-8.3457 ;-6.2234)$ & 1.0032 & - \\
$\mu_{2}$ & 0.0924 & 0.2339 & $(-0.3536 ; 0.5713)$ & 1.0014 & - \\
$\sigma_{1}^{2}$ & 0.0448 & 0.3570 & $(0.0167 ; 0.0724)$ & 1.0936 & - \\
$\sigma_{2}^{2}$ & 1.3606 & 0.4320 & $(0.7312 ; 2.3887)$ & 1.0041 & - \\
$p_{1}$ & 0.0868 & 0.0473 & $(0.0183 ; 0.1964)$ & 1.0006 & - \\
$\beta_{11}$ & 0.1459 & 0.1705 & $(-0.1988 ; 0.4813)$ & 0.9993 & 0.7519 \\
$\beta_{12}$ & 0.4860 & 0.1740 & $(0.1313 ; 0.8213)$ & 1.0022 & 0.7039 \\
$\beta_{13}$ & 0.3197 & 0.1733 & $(-0.0301 ; 0.6526)$ & 1.0015 & 0.7869 \\
$\beta_{14}$ & -0.1112 & 0.1764 & $(-0.4703 ; 0.2269)$ & 0.9999 & 0.6261 \\
\hline \hline
\end{tabular}


Do modelo ajustado pelos parâmetros dados na tabela 6.26 calculamos os resíduos de Pearson $d_{i j}$ (5.2) dados na tabela 6.27.

Tabela 6.27: Resíduos de Pearson (mistura de duas distribuições normais para $\alpha_{i}$ )

\begin{tabular}{|c|c|c|c|}
\hline & & & \\
\hline 1 & 2 & 3 & 4 \\
\hline-0.0266 & -0.0266 & -0.0266 & -0.0266 \\
\hline-0.6014 & -0.6014 & -0.6014 & 1.6629 \\
\hline-0.5934 & -0.5934 & 1.6853 & -0.5934 \\
\hline-1.0018 & -1.0018 & 0.9983 & 0.9983 \\
\hline-0.5950 & 1.6807 & -0.5950 & -0.5950 \\
\hline-1.0172 & 0.9830 & -1.0172 & 0.9830 \\
\hline-1.0046 & 0.9955 & 0.9955 & -1.0046 \\
\hline-1.5859 & 0.6306 & 0.6306 & 0.6306 \\
\hline 1.7014 & -0.5878 & -0.5878 & -0.5878 \\
\hline 0.9928 & -1.0073 & -1.0073 & 0.9928 \\
\hline 0.9865 & -1.0136 & 0.9865 & -1.0136 \\
\hline 0.6598 & -1.5157 & 0.6598 & 0.6598 \\
\hline 0.9864 & 0.9864 & -1.0138 & -1.0138 \\
\hline 0.6681 & 0.6681 & -1.4968 & 0.6681 \\
\hline 0.6270 & 0.6270 & 0.6270 & -1.5950 \\
\hline 0.2257 & 0.2257 & 0.2257 & 0.2257 \\
\hline-0.0288 & -0.0341 & -0.0314 & -0.0253 \\
\hline-0.6061 & -0.7185 & -0.6611 & 1.8762 \\
\hline-0.5929 & -0.7029 & 1.5461 & -0.5214 \\
\hline-1.0311 & -1.2222 & 0.8891 & 1.1029 \\
\hline-0.5807 & 1.4527 & -0.6334 & -0.5107 \\
\hline-1.0410 & 0.8104 & -1.1355 & 1.0924 \\
\hline-0.9816 & 0.8595 & 0.9340 & -0.8632 \\
\hline-1.5903 & 0.5305 & 0.5765 & 0.7151 \\
\hline 1.6912 & -0.7009 & -0.6450 & -0.5200 \\
\hline 0.9988 & -1.1868 & -1.0921 & 1.1358 \\
\hline 1.0024 & -1.1826 & 0.9189 & -0.8773 \\
\hline 0.7028 & -1.6867 & 0.6443 & 0.7992 \\
\hline 1.0011 & 0.8446 & -1.0896 & -0.8784 \\
\hline 0.6484 & 0.5470 & -1.6824 & 0.7373 \\
\hline 0.6314 & 0.5327 & 0.5789 & -1.3927 \\
\hline 0.2605 & 0.2198 & 0.2388 & 0.2962 \\
\hline
\end{tabular}

$\mathrm{Na}$ figura 6.17, apresentamos os gráficos dos resíduos de Pearson $d_{i j}$ 


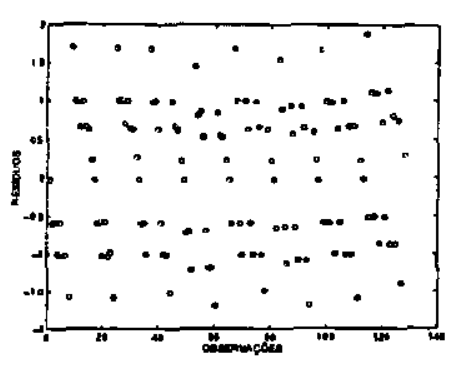

$j=1, \ldots, 4$

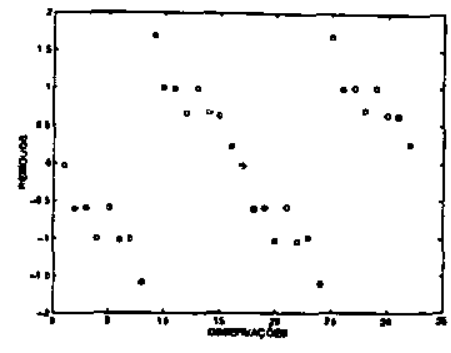

$j=1$

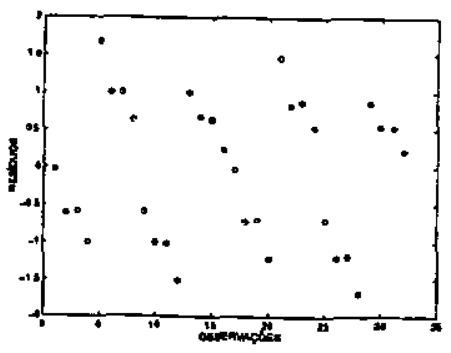

$j=2$

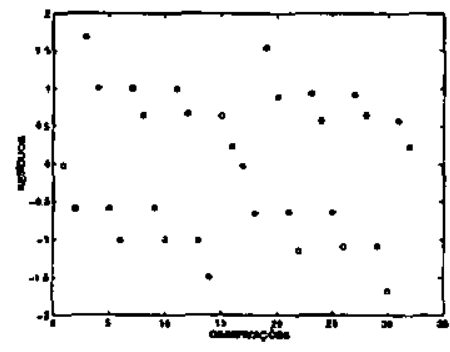

$j=3$

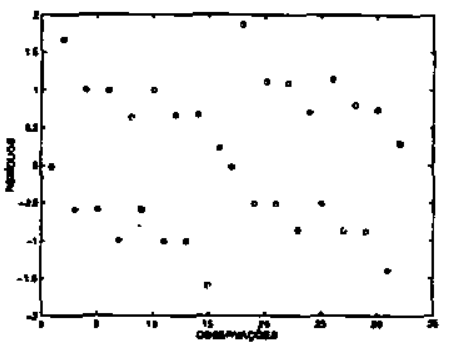

$j=4$

Figura 6.17: Resíduos de Pearson assumindo uma mistura de normais para os efeitos aleatórios

\subsubsection{Comparação dos Modelos 7, 8 e 9}

Na tabela 6.28, temos um resumo dos estimadores de Monte Carlo para a média a posteriori dos parâmetros $\beta_{1 j}(j=1,2,3,4)$ baseados em amostras geradas pelo algoritmo Metropolis-Hastings considerando os diferentes modelos com função de ligação logística (modelos 7, 8 e 9), onde pode ser observado uma variação dos estimadores entre os diferentes modelos.

Tabela 6.28: Sumário à Posteriori para $\beta_{1 j}, j=1,2,3,4$

\begin{tabular}{crrrr}
\hline Parâmetro & EMV & $\alpha$ fixo & $\alpha_{i} \sim N\left(0, \sigma_{\alpha}^{2}\right)$ & $\alpha_{i} \sim$ Mistura de Normais \\
\hline \hline$\beta_{11}$ & 0.2054 & 0.2022 & 0.1935 & 0.1459 \\
$\beta_{12}$ & 0.4876 & 0.3990 & 0.4032 & 0.4860 \\
$\beta_{13}$ & 0.3527 & 0.2953 & 0.2940 & 0.3197 \\
$\beta_{14}$ & -0.0021 & -0.0043 & -0.0058 & -0.1112 \\
\hline \hline
\end{tabular}

Para compararmos a eficiência dos modelos, na tabela 6.29 temos o Total de Pearson dado por (5.3) e também os valores das densidades preditivas nos pontos observados, que por dificuldades numéricas nestes modelos que envolvem réplicas, tivemos que optar pela aproximação (5.8) para a densidade preditiva no lugar da aproximação de média harmônica dada por (5.9). Isso é devido a algumas frequências terem muito peso sobre 
a verossimilhança, o que computacionalmente leva algumas verossimilhança para infinito ou para zero. Utilizando a estimativa (5.8) a verossimilhança preditiva é dada por,

$$
\begin{aligned}
\widehat{p}_{1}\left(\widehat{y} \mid M_{k}\right) & =\frac{1}{m} \sum_{t=1}^{m} \mathrm{~L}\left(d \mid \underset{\sim}{\alpha^{(t)}} \underset{\sim_{1}^{\beta^{(t)}}}{\sim_{1}}\right. \\
& =\frac{1}{m} \sum_{t=1}^{m}\left[\prod_{i=1}^{32} \prod_{j=1}^{4}\left(\frac{\exp \left\{\left(\alpha_{i}^{(t)}+x_{i j 1} \beta_{1 j}^{(t)}\right) y_{i j}\right\}}{1+\exp \left\{\alpha_{i}^{(t)}+x_{i j 1} \beta_{1 j}^{(t)}\right\}}\right)^{f_{i}}\right]
\end{aligned}
$$

onde $k=7,8,9$, indica o modelo o qual esta sendo calculada a densidade preditiva e $m$ o tamanho da amostra gerada.

Tabela 6.29: Resultados do Total de Pearson (6.4) e para as Densidades Preditivas para os Modelos Calculados nos Valores Observados

\begin{tabular}{c|ccc}
\hline & Modelo 7 & Modelo 8 & Modelo 9 \\
\hline$D(32 \mathrm{obs})$ & 359.7745 & 110.3901 & 109.9357 \\
$\widehat{p}_{1}\left(\widehat{y} \mid M_{k}\right)(32 \mathrm{obs})$ & - & $2.0669 \cdot 10^{-180}$ & $1.4616 \cdot 10^{-175}$ \\
\hline \hline
\end{tabular}

Notamos pela tabela 6.29, uma grande diferença do Total de Pearson entre o modelo 7 e os modelos 8 e 9, mostrando que os modelos de efeitos aleatórios são melhores havendo uma superioridade para o modelo 9. Superioridade esta que fica muito acentuada quando observamos as densidades preditivas, pois $B_{98}=7.0715 \cdot 10^{4}$. No modelo 7 as verossimilhanças foram muito baixas impossibilitando o seu cálculo.

Nas figuras 6.18 e 6.19 apresentamos dois gráficos de frequências para os $\alpha_{i}, i=$ $1,2, \ldots, 32$ para os modelos de efeitos aleatórios, modelos 8 e 9 respectivamente. 


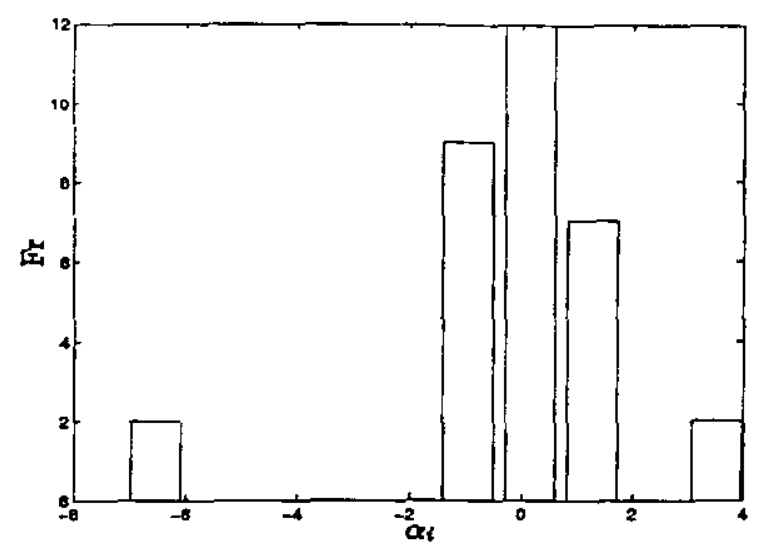

Figura 6.18: Frequências dos $\alpha_{i}$ Assumindo $\alpha_{i} \stackrel{i i d}{\sim} N\left(0, \sigma_{\alpha}^{2}\right) i=1,2, \ldots, 32$

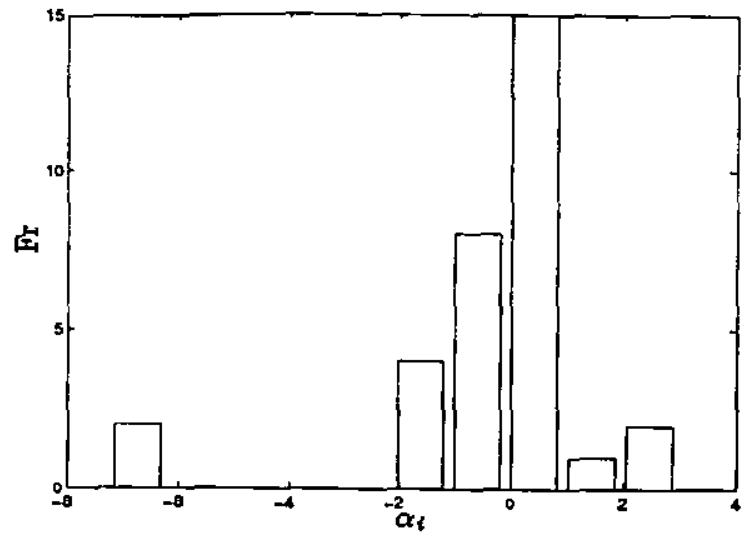

Figura 6.19: Frequências dos $\alpha_{i}$ Assumindo $\alpha_{i}, i=1,2, \ldots, 32$ Mistura de duas Normais

\subsubsection{EMV para os Parâmetros do Modelo com Ligação Probito Considerando Réplicas}

$\mathrm{Na}$ tabela 6.30 apresentamos os estimadores de máxima verossimilhança e os intervalos de confiança assintóticos para os parâmetros do modelo probito assumindo efeitos fixos e réplicas.

Tabela 6.30: Estimadores de máxima verosimilhança

\begin{tabular}{ccc}
\hline Parâmetro & EMV & Int. Conf. (95\%) \\
\hline$\alpha$ & -1.0835 & $(-1.1463 ;-1.0208)$ \\
$\beta_{11}$ & 0.1125 & $(-0.1079 ; 0.3330)$ \\
$\beta_{12}$ & 0.2721 & $(0.0221 ; 0.5221)$ \\
$\beta_{13}$ & 0.1951 & $(-0.0389 ; 0.4292)$ \\
$\beta_{14}$ & -0.0011 & $(-0.2075 ; 0.2053)$ \\
\hline
\end{tabular}

\subsubsection{Aplicação do Modelo 10}

Considerando os dados introduzidos por Chib e Greenberg (1998), e o modelo probito de efeitos fixos com réplicas, escolhemos os seguintes valores para as densidades a priori $\mu_{0}=-1, \sigma_{0}=0.3, b_{11}=0.1, b_{12}=0.2, b_{13}=0.1, b_{14}=0$, e $c_{1 j}=0.3, j=1,2,3,4$.

$\mathrm{Na}$ tabela 6.31, apresentamos os resumos a posteriori correspondentes aos parâmetros do modelo 10, a partir das distribuições condicionais das quais geramos 5 cadeias de 
Metropolis-Hastings de 6000 pontos cada, todas as cadeias com condições iniciais diferentes, desprezamos as 3000 primeiras iterações, a partir destas tomamos as $3015^{\underline{a}}, 3030^{a}$, ... a fim de dimimuir a auto correlação entre as amostras, formando assim amostras de 1000 pontos para cada parâmetro. Podemos considerar a convergência para as distribuições de equilíbrio, pois pela tabela $6.31 \sqrt{\widehat{R}}<1.1$.

Tabela 6.31: Sumário à Posteriori considerando $\alpha_{i}=\alpha$

\begin{tabular}{cccccc}
\hline Parâmetro & Média & DP & IC 95\% & $\sqrt{\widehat{R}}$ & TAc \\
\hline \hline$\alpha$ & -1.0036 & 0.2917 & $(-1.5528 ;-0.4330)$ & 1.0010 & 0.9994 \\
$\beta_{11}$ & 0.1241 & 0.2835 & $(-0.4636 ; 0.6657)$ & 0.9996 & 0.9545 \\
$\beta_{12}$ & 0.1636 & 0.3156 & $(-0.4764 ; 0.7805)$ & 1.0024 & 0.9592 \\
$\beta_{13}$ & 0.0867 & 0.3159 & $(-0.5396 ; 0.6894)$ & 1.0034 & 0.9524 \\
$\beta_{14}$ & 0.0209 & 0.2838 & $(-0.5301 ; 0.5662)$ & 1.0049 & 0.9506 \\
\hline \hline
\end{tabular}

A seguir apresentamos (figura 6.20 e na tabela 6.32) os resíduos de Pearson $d_{i j}$ (5.2), que expressa a medida de discrepância entre os dados e o modelo 10.

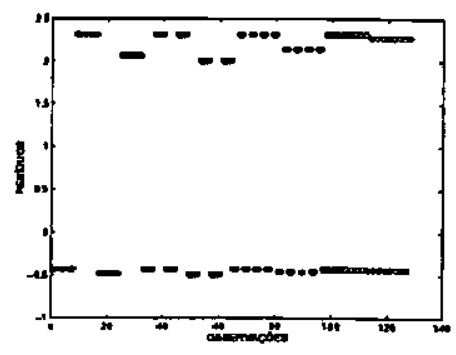

$j=1, \ldots, 4$

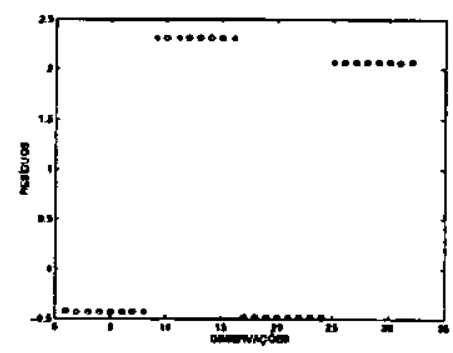

$j=1$

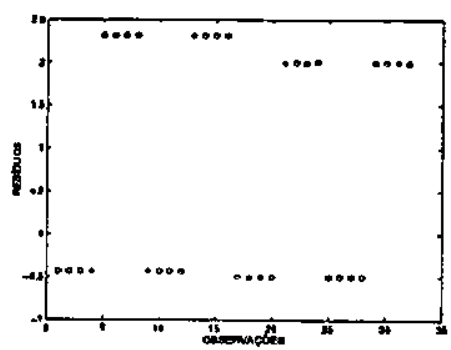

$j=2$

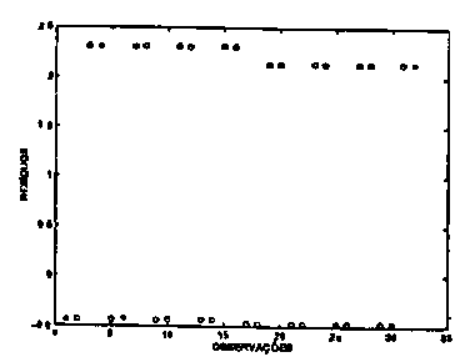

$j=3$

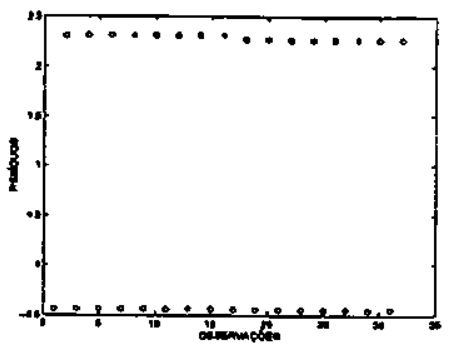

$j=4$

Figura 6.20: Resíduos de Pearson considerando $\alpha_{i}=\alpha(i=1,2, \ldots, 32)$ 
Tabela 6.32: Resíduos de Pearson assumindo

\begin{tabular}{|c|c|c|c|}
\hline \multicolumn{4}{|c|}{$\alpha_{i}=\alpha(i=1,2, \ldots, 32)$} \\
\hline \multicolumn{4}{|c|}{$\mathbf{J}$} \\
\hline 1 & 2 & 3 & 4 \\
\hline-0.4328 & -0.4328 & -0.4328 & -0.4328 \\
\hline-0.4328 & -0.4328 & -0.4328 & 2.3103 \\
\hline-0.4328 & -0.4328 & 2.3103 & -0.4328 \\
\hline-0.4328 & -0.4328 & 2.3103 & 2.3103 \\
\hline-0.4328 & 2.3103 & -0.4328 & -0.4328 \\
\hline-0.4328 & 2.3103 & -0.4328 & 2.3103 \\
\hline-0.4328 & 2.3103 & 2.3103 & -0.4328 \\
\hline-0.4328 & 2.3103 & 2.3103 & 2.3103 \\
\hline 2.3103 & -0.4328 & -0.4328 & -0.4328 \\
\hline 2.3103 & -0.4328 & -0.4328 & 2.3103 \\
\hline 2.3103 & -0.4328 & 2.3103 & -0.4328 \\
\hline 2.3103 & -0.4328 & 2.3103 & 2.3103 \\
\hline 2.3103 & 2.3103 & -0.4328 & -0.4328 \\
\hline 2.3103 & 2.3103 & -0.4328 & 2.3103 \\
\hline 2.3103 & 2.3103 & 2.3103 & -0.4328 \\
\hline 2.3103 & 2.3103 & 2.3103 & 2.3103 \\
\hline-0.4836 & -0.5007 & -0.4679 & -0.4411 \\
\hline-0.4836 & -0.5007 & -0.4679 & 2.2671 \\
\hline-0.4836 & -0.5007 & 2.1373 & -0.4411 \\
\hline-0.4836 & -0.5007 & 2.1373 & 2.2671 \\
\hline-0.4836 & 1.9972 & -0.4679 & -0.4411 \\
\hline-0.4836 & 1.9972 & -0.4679 & 2.2671 \\
\hline-0.4836 & 1.9972 & 2.1373 & -0.4411 \\
\hline-0.4836 & 1.9972 & 2.1373 & 2.2671 \\
\hline 2.0677 & -0.5007 & -0.4679 & -0.4411 \\
\hline 2.0677 & -0.5007 & -0.4679 & 2.2671 \\
\hline 2.0677 & -0.5007 & 2.1373 & -0.4411 \\
\hline 2.0677 & -0.5007 & 2.1373 & 2.2671 \\
\hline 2.0677 & 1.9972 & -0.4679 & -0.4411 \\
\hline 2.0677 & 1.9972 & -0.4679 & 2.2671 \\
\hline 2.0677 & 1.9972 & 2.1373 & -0.4411 \\
\hline 2.0677 & 1.9972 & 2.1373 & 2.2671 \\
\hline
\end{tabular}

\subsubsection{Aplicação do Modelo 11}

Para as densidades a priori (4.9) escolhemos os seguintes valores: $a=3, b=4 \mathrm{e}$ os $b_{1 j}$ e $c_{1 j}, j=1,2,3,4$ como sendo os mesmos usados no modelo 10 . 
Na tabela 6.33 apresentamos o sumário a posteriori para os parâmetros do modelo 5, a partir das amostras geradas das distribuições condicionais a posteriori. Para obtermos este sumário geramos 5 cadeias de 6000 iterações e consideramos uma amostra final de 1000 pontos amostrais, que foi suficiente para verificar a convergência observando que para todos os parâmetros $\sqrt{\widehat{R}}<1.1$.

Tabela 6.33: Sumário à Posteriori considerando $\alpha_{i} \sim N\left(0, \sigma_{\alpha}^{2}\right)$

\begin{tabular}{crcccc}
\hline Parâmetro & Média & DP & IC 95\% & $\sqrt{\widehat{R}}$ & TAc \\
\hline \hline$\sigma_{\alpha}^{2}$ & 1.0688 & 0.1390 & $(0.8163 ; 1.2866)$ & 1.0553 & 1.0000 \\
$\beta_{11}$ & 0.0034 & 0.1899 & $(-0.3839 ; 0.3583)$ & 1.0148 & 0.5117 \\
$\beta_{12}$ & 0.2969 & 0.1986 & $(-0.0930 ; 0.6460)$ & 1.0158 & 0.5002 \\
$\beta_{13}$ & 0.1391 & 0.1941 & $(-0.2427 ; 0.5039)$ & 1.0126 & 0.5235 \\
$\beta_{14}$ & -0.1976 & 0.1859 & $(-0.5840 ; 0.1451)$ & 1.0053 & 0.4471 \\
\hline \hline
\end{tabular}

A partir do modelo ajustado pelos parâmetros dados na tabela 6.33 calculamos os resíduos de Pearson $d_{i j}$ (5.2) dados na tabela 6.34 . 
Table 6.34: Resíduos de Pearson $\left(\alpha_{i} \sim N\left(0, \sigma_{\alpha}^{2}\right)\right)$

\begin{tabular}{|c|c|c|c|}
\hline \multicolumn{4}{|c|}{$\mathrm{J}$} \\
\hline 1 & 2 & 3 & 4 \\
\hline-0.0294 & -0.0294 & -0.0294 & -0.0294 \\
\hline-0.5890 & -0.5890 & -0.5890 & 1.6978 \\
\hline-0.5823 & -0.5823 & 1.7173 & -0.5823 \\
\hline-0.9913 & -0.9913 & 1.0088 & 1.0088 \\
\hline-0.5846 & 1.7107 & -0.5846 & -0.5846 \\
\hline-1.0077 & 0.9924 & -1.0077 & 0.9924 \\
\hline-1.0054 & 0.9946 & 0.9946 & -1.0054 \\
\hline-1.6286 & 0.6140 & 0.6140 & 0.6140 \\
\hline 0.8729 & -1.1456 & -1.1456 & -1.1456 \\
\hline 0.9920 & -1.0081 & -1.0081 & 0.9920 \\
\hline 0.9911 & -1.0090 & 0.9911 & -1.0090 \\
\hline 0.6311 & -1.5846 & 0.6311 & 0.6311 \\
\hline 1.0014 & 1.0014 & -0.9986 & -0.9986 \\
\hline 0.6319 & 0.6319 & -1.5825 & 0.6319 \\
\hline 0.5979 & 0.5979 & 0.5979 & -1.6724 \\
\hline 0.1137 & 0.1137 & 0.1137 & 0.1137 \\
\hline-0.0265 & -0.0432 & -0.0334 & -0.0185 \\
\hline-0.5768 & -0.7357 & -0.6462 & 2.0626 \\
\hline-0.5636 & -0.7195 & 1.5828 & -0.4733 \\
\hline-0.9750 & -1.2331 & 0.9203 & 1.2046 \\
\hline-0.5413 & 1.4444 & -0.6074 & -0.4539 \\
\hline-0.9785 & 0.8081 & -1.0904 & 1.2003 \\
\hline-0.9607 & 0.8231 & 0.9340 & -0.8179 \\
\hline-1.6251 & 0.4782 & 0.5486 & 0.7264 \\
\hline 1.7620 & -0.7244 & -0.6361 & -0.4768 \\
\hline 1.0445 & -1.2107 & -1.0670 & 1.2269 \\
\hline 1.0405 & -1.2153 & 0.9337 & -0.8182 \\
\hline 0.6885 & -1.8580 & 0.6153 & 0.8107 \\
\hline 1.0450 & 0.8264 & -1.0664 & -0.8146 \\
\hline 0.6511 & 0.5075 & -1.7207 & 0.7675 \\
\hline 0.6125 & 0.4759 & 0.5460 & -1.3829 \\
\hline 0.1496 & 0.1028 & 0.1263 & 0.1897 \\
\hline
\end{tabular}




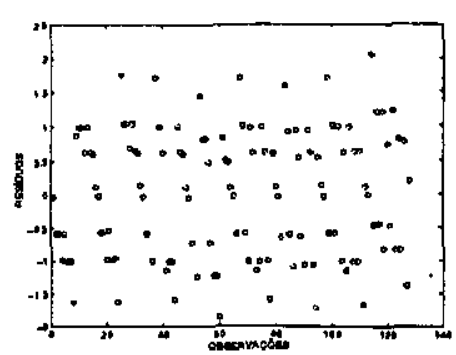

$j=1, \ldots, 4$

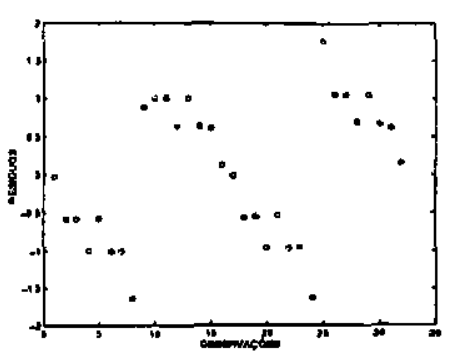

$j=1$

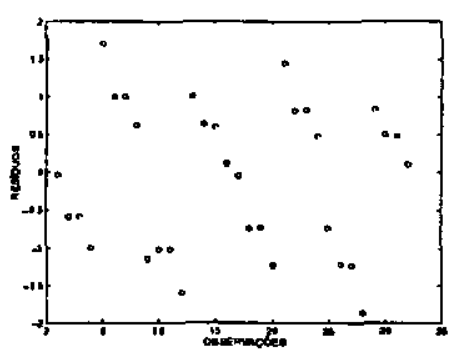

$j=2$

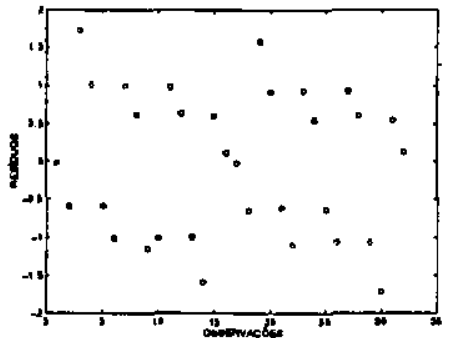

$j=3$

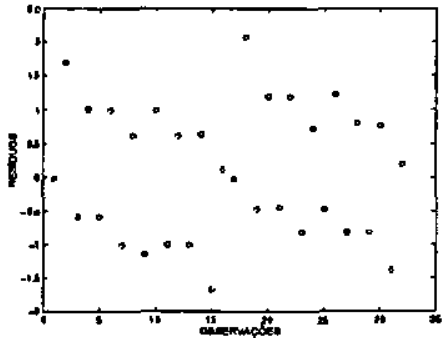

$j=4$

Figura 6.21: Resíduos de Pearson considerando $\alpha_{i} \sim N\left(0, \sigma_{\alpha}^{2}\right), i=1,2, \ldots, 32$

\subsubsection{Aplicação do Modelo 12}

Numa análise Bayesiana do modelo probito assumindo uma mistura de distribuições normais com réplicas para os efeitos aleatórios $\alpha_{i}, i=1,2, \ldots, 36$, consideramos os seguintes valores para as densidades a priori (4.11): $d_{1}=-0.1, d_{2}=0.3, e_{1}=e_{1}=0.2$, $a_{1}=a_{2}=4, b_{1}=b_{2}=3, f=1, g=1$ e os valores para $b_{l j}$ e $c_{l j}, l=1,2,3 ; j=1,2, \ldots, 6$ os mesmos usados nos modelos 4 e 5 .

Os resumos a posteriori sāo apresentados na tabela 6.35 , a seguir.

Tabela 6.35: Sumário à Posteriori considerando $\alpha_{i}$ com Mistura de Normais

\begin{tabular}{cccccc}
\hline Parâmetro & Média & DP & IC 95\% & $\sqrt{\widehat{R}}$ & TAc \\
\hline$\mu_{2}$ & -0.5460 & 0.4709 & $(-1.5895 ; 0.2569)$ & 0.9993 & 1.0000 \\
$\mu_{2}$ & 0.2107 & 0.3444 & $(-0.4219 ; 0.9332)$ & 1.0022 & 1.0000 \\
$\sigma_{1}^{2}$ & 1.0390 & 0.1449 & $(0.8085 ; 1.2871)$ & 1.0023 & 1.0000 \\
$\sigma_{2}^{2}$ & 1.0164 & 0.1431 & $(0.8070 ; 1.2822)$ & 0.9993 & 1.0000 \\
$p_{1}$ & 0.4360 & 0.2747 & $(0.0318 ; 0.9570)$ & 0.9999 & 1.0000 \\
$\beta_{11}$ & 0.0222 & 0.1868 & $(-0.3522 ; 0.3843)$ & 1.0058 & 0.5091 \\
$\beta_{12}$ & 0.3155 & 0.1868 & $(-0.0393 ; 0.6887)$ & 1.0085 & 0.4730 \\
$\beta_{13}$ & 0.1686 & 0.1871 & $(-0.2096 ; 0.5404)$ & 1.0073 & 0.5096 \\
$\beta_{14}$ & -0.1679 & 0.1776 & $(-0.5121 ; 0.1715)$ & 1.0056 & 0.4635 \\
\hline \hline
\end{tabular}

Na tabela 6.36 apresentamos o resíduo de Pearson $d_{i j}$. 
Tabela 6.36: Resíduos de Pearson (mistura de duas distribuições normais para $\alpha_{i}$ )

\begin{tabular}{|c|c|c|c|}
\hline \multicolumn{4}{|c|}{$\mathrm{J}$} \\
\hline 1 & 2 & 3 & 4 \\
\hline-0.0213 & -0.0213 & -0.0213 & -0.0213 \\
\hline-0.5778 & -0.5778 & -0.5778 & 1.7306 \\
\hline-0.5846 & -0.5846 & 1.7107 & -0.5846 \\
\hline-0.9940 & -0.9940 & 1.0060 & 1.0060 \\
\hline-0.5804 & 1.7228 & -0.5804 & -0.5804 \\
\hline-1.0080 & 0.9921 & -1.0080 & 0.9921 \\
\hline-1.0022 & 0.9978 & 0.9978 & -1.0022 \\
\hline-1.6277 & 0.6144 & 0.6144 & 0.6144 \\
\hline 1.7178 & -0.5822 & -0.5822 & -0.5822 \\
\hline 1.0128 & -0.9874 & -0.9874 & 1.0128 \\
\hline 1.0180 & -0.9824 & 1.0180 & -0.9824 \\
\hline 0.6506 & -1.5370 & 0.6506 & 0.6506 \\
\hline 0.9985 & 0.9985 & -1.0015 & -1.0015 \\
\hline 0.6433 & 0.6433 & -1.5545 & 0.6433 \\
\hline 0.5934 & 0.5934 & 0.5934 & -1.6853 \\
\hline 0.1077 & 0.1077 & 0.1077 & 0.1077 \\
\hline-0.0265 & -0.0432 & -0.0340 & -0.0189 \\
\hline-0.5714 & -0.7289 & -0.6460 & 2.0629 \\
\hline-0.5486 & -0.7011 & 1.6106 & -0.4647 \\
\hline-0.9370 & -1.1845 & 0.9495 & 1.2429 \\
\hline-0.5384 & 1.4522 & -0.6096 & -0.4557 \\
\hline-0.9424 & 0.8394 & -1.0591 & 1.2358 \\
\hline-0.9513 & 0.8314 & 0.9352 & -0.8170 \\
\hline-1.6089 & 0.4834 & 0.5492 & 0.7270 \\
\hline 1.7914 & -0.7129 & -0.6315 & -0.4732 \\
\hline 1.0552 & -1.1981 & -1.0652 & 1.2287 \\
\hline 1.0474 & -1.2071 & 0.9319 & -0.8200 \\
\hline 0.6996 & -1.8268 & 0.6198 & 0.8163 \\
\hline 1.0600 & 0.8385 & -1.0603 & -0.8101 \\
\hline 0.6490 & 0.5059 & -1.7421 & 0.7584 \\
\hline 0.6186 & 0.4810 & 0.5465 & -1.3819 \\
\hline 0.1419 & 0.0970 & 0.1179 & 0.1782 \\
\hline
\end{tabular}




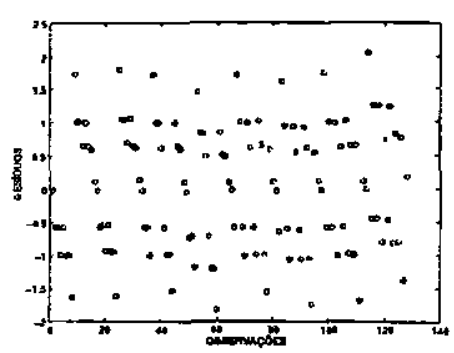

$j=1, \ldots, 4$

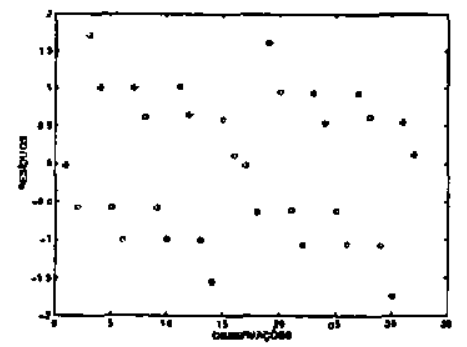

$j=3$

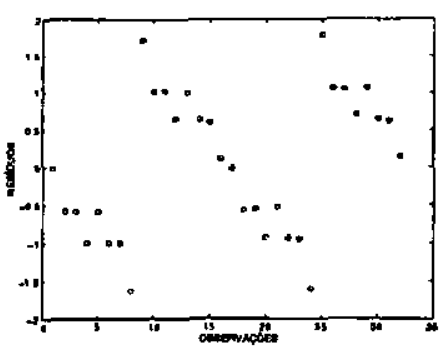

$j=1$

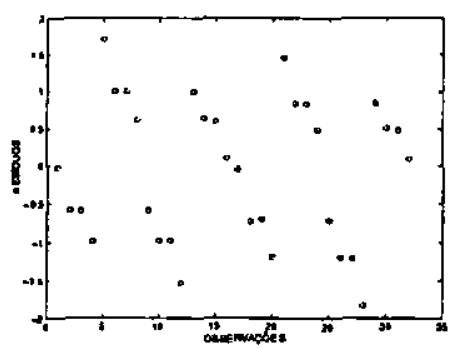

$j=2$

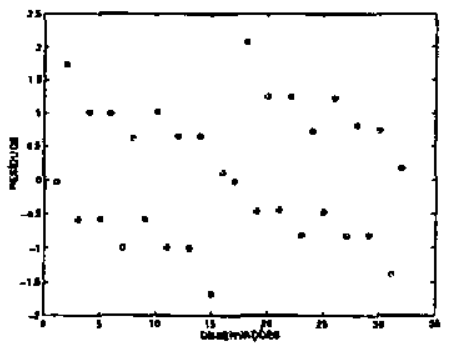

$j=4$

Figura 6.22: Resíduos de Pearson assumindo uma mistura de normais para os efeitos aleatórios

\subsubsection{Comparação dos Modelos 10, 11 e 12}

$\mathrm{Na}$ tabela 6.37 apresentamos os estimadores a posteriori para os parâmetros dos modelos 10, 11, 12 e os estimadores de máxima verossimilhança.

\begin{tabular}{crrrr}
\multicolumn{6}{l}{ Tabela 6.37: Sumário à Posteriori para $\beta_{1 j}, j=1,2,3,4$} \\
Parâmetro & EMV & $\alpha$ fixo & $\alpha_{i} \sim N\left(0, \sigma_{\alpha}^{2}\right)$ & $\alpha_{i}$ Mistura de Normais \\
\hline \hline$\beta_{11}$ & 0.1125 & 0.1241 & 0.0034 & 0.0222 \\
$\beta_{12}$ & 0.2721 & 0.1636 & 0.2969 & 0.3155 \\
$\beta_{13}$ & 0.1951 & 0.0867 & 0.1391 & 0.1686 \\
$\beta_{14}$ & -0.0011 & 0.0209 & -0.1976 & -0.1679 \\
\hline \hline
\end{tabular}

Na tabela 6.38, apresentamos o Total de Pearson (5.3) e as densidades preditivas calculadas nas observações para checagem da performance dos modelos.

Tabela 6.38: Resultados do Total de Pearson (6.4) e as Densidades Preditivas para os Modelos

\begin{tabular}{c|ccc}
\hline & Modelo 10 & Modelo 11 & Modelo 12 \\
\hline \hline$D(32$ obs $)$ & 327.7585 & 112.8056 & 112.0882 \\
$\widehat{p}_{1}\left(\widehat{y} \mid M_{k}\right)(32$ obs $)$ & - & $8.9319 \cdot 10^{-175}$ & $1.0092 \cdot 10^{-174}$ \\
\hline \hline
\end{tabular}


Observamos que o Total de Pearson não revela diferença entre os modelos 11 e 12, mas as densidades preditivas apresentam uma grande diferença entre os modelos evidênciando uma melhor performance do modelo probito de efeitos aleatórios com uma mistura de duas distribuiçōes normais.

A seguir temos nas figura 6.23 e 6.24 , histogramas que mostram as frequências dos $\alpha_{i}, i=1,2, \ldots, 36$ para os modelos 5 e 6 respectivamente.

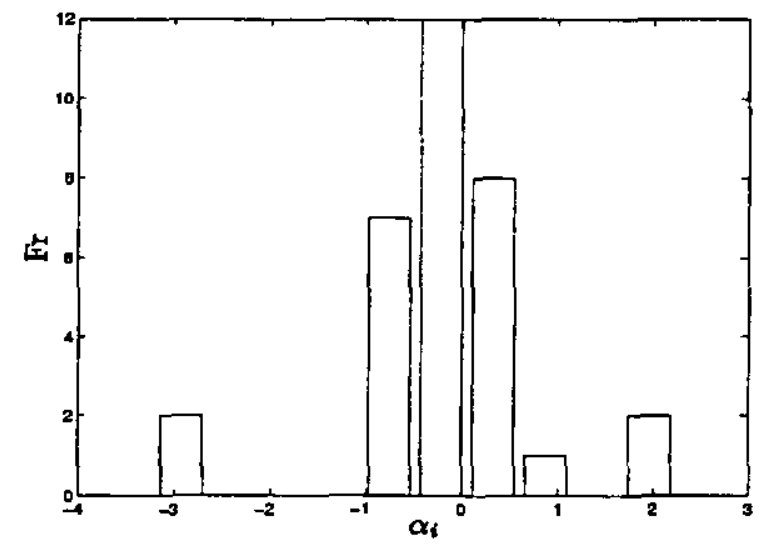

Figura 6.23: Frequências dos $\alpha_{i}$ Assumindo $\alpha_{i} \stackrel{i i d}{\sim} N\left(0, \sigma_{\alpha}^{2}\right) i=1,2, \ldots, 32$

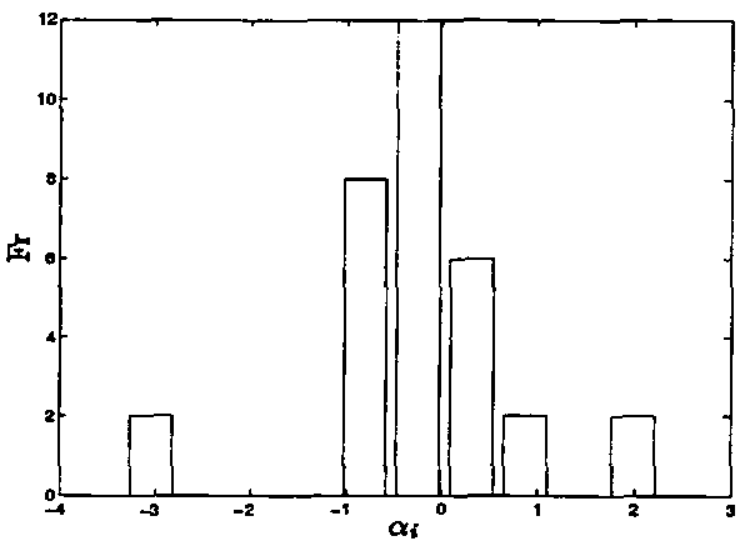

Figura 6.24: Frequências dos $\alpha_{i}$ Assumindo $\alpha_{i}, i=1,2, \ldots, 32$ Mistura de duas Normais 


\section{Capítulo 7}

\section{CONCLUSÕES E \\ CONSIDERAÇÕES FUTURAS}

Nesta dissertação, observamos que o uso de mistura de distribuiçōes normais para modelos de efeitos aleatórios para dados binários correlacionados, considerando as funçōes de ligações logito e probito apresentam uma grande flexibilidade para o ajuste do modelo aos dados reais.

Usualmente, em experimentos envolvendo dados binários correlacionados, existem várias covariáveis de interesse, o que acarreta em um grande número de parâmetros e neste caso verificamos que o uso de métodos Bayesianos com técnicas de simulação de Monte Carlo em Cadeias de Markov (MCMC), são apropriadas para a obtenção de sumários a posteriori de interesse.

Fazendo uma comparação entre as funções de ligação logística e probito podemos verificar através do Total de Pearson e das densidades preditivas que a função de ligação logística apresentou uma melhor performance para os dados considerados entre os modelos de mesma ordem, tanto para dados replicados quanto para dados sem réplicas. Do ponto de vista computacional constatamos que a função de ligaçāo probito apresenta maiores dificuldades em sua implementação pelo fato de convergir para 0 ou 1 (zero ou um) quando se trata valores absolutos menores que os necessários que isso ocorra na função de ligaçāo 
logística. Na figura 7.1, observamos esse comportamento.

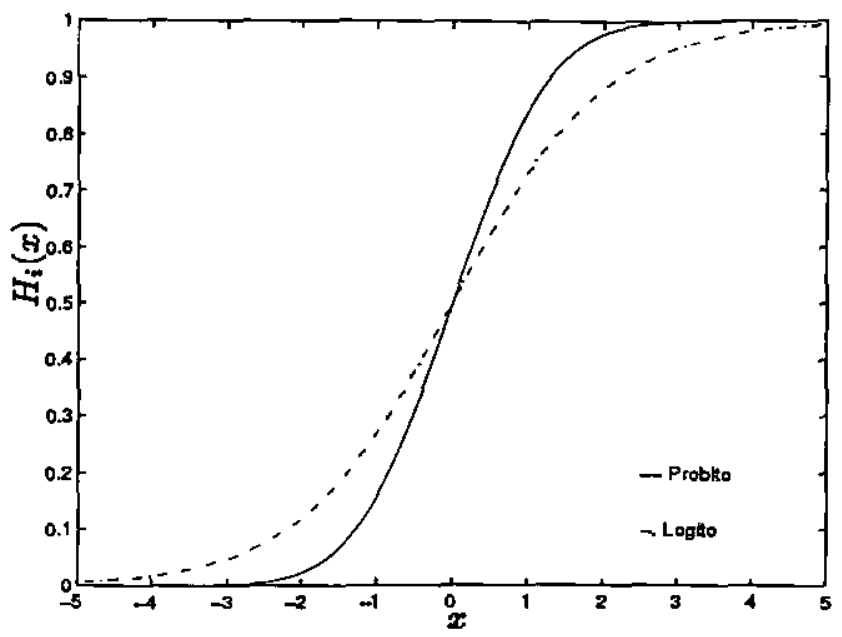

Figura 7.1: Comparação do Comportamento entre as Funções de Ligação Logito e Probito

Na figura 7.1 temos que $H_{i}(x), i=1,2$, é dado por;

$$
\begin{aligned}
& H_{1}(x)=\Phi(x) \text { (Probito) } \\
& H_{2}(x)=\frac{e^{x}}{1+e^{x}} \text { (Logito) }
\end{aligned}
$$

Para desenvolvimento futuro, podemos considerar o uso dos modelos condicionais e multivariados como uma ligaçāo $t$-de-Student sob o enfoque Bayesiano, assumindo os aspectos computacionais considerados neste trabalho. 


\section{Apêndice A}

\section{ALGORITMO GIBBS SAMPLING}

O algoritmo Gibbs sampling foi introduzido formalmente por Geman e Geman (1984), quando propuseram um esquema de amostragem de distribuiçōes explorando as distribuições condicionais completas através de um algoritmo iterativo que define uma cadeia de Markov.

O algoritmo Gibbs sampling é essencialmente um esquema iterativo de amostragem de uma cadeia de Markov cujo núcleo de transição é formado pelas distribuiçōes condicionais completas.

Descrição do método:

Suponha que a distribuição de interesse é $\pi(\theta)$ onde $\theta=\left(\theta_{1}, \theta_{2}, \ldots, \theta_{p}\right)$. Considere ainda que as densidades condicionais completas a posteriori $\pi_{i}\left(\theta_{i} \mid \theta_{-i}\right), \quad i=1, \ldots, p$ estão disponíveis sob forma padronizada. Podemos então gerar as amostras como segue:

(i) inicializemos o contador de iterações da cadeia $j=1$ e arbitremos valores iniciais $\theta^{(0)}=\theta_{1}^{(0)}, \theta_{2}^{(0)}, \ldots, \theta_{p}^{(0)}$.

(ii) obtemos um novo valor $\theta^{(j)}=\theta_{1}^{(j)}, \theta_{2}^{(j)}, \ldots, \theta_{p}^{(j)}$ a partir de $\theta^{(j-1)}$ através de sucessivas gerações de valores

$$
\begin{aligned}
\theta_{1}^{(j)} & \sim \pi\left(\theta_{1} \mid \theta_{2}^{(j-1)}, \ldots, \theta_{p}^{(j-1)}\right) \\
\theta_{2}^{(j)} & \sim \pi\left(\theta_{2} \mid \theta_{1}^{(j)}, \theta_{3}^{(j-1)}, \ldots, \theta_{p}^{(j-1)}\right) \\
\quad & \\
\theta_{p}^{(j)} & \sim \pi\left(\theta_{p} \mid \theta_{1}^{(j)}, \theta_{2}^{(j)}, \ldots, \theta_{p-1}^{(j)}\right)
\end{aligned}
$$

(iii) mudamos o contador $j$ para $j+1$ e retornamos a (ii) até a convergência. 
Geman e Geman (1984) mostram que os valores gerados para $\theta_{i}, \quad i=1, \ldots, p$, convergem em distribuição para uma variável aleatória da distribuição $\pi(\theta) \operatorname{com} j \longrightarrow \infty$. 


\section{Apêndice B}

\section{ALGORITMO DE}

\section{METROPOLIS-HASTINGS}

Este algoritmo é usado quando as densidades condicionais não são dadas sob forma padronizada conhecida, como de uma Normal, Gama ou outras.

Para descrevermos este método consideremos uma distribuição $\pi$, com densidades condicionais não padronizadas, da qual desejamos gerar uma amostra através de uma cadeia de Markov. Nesse caso, devemos construir um núcleo de transição $p(\theta, \phi)$ de forma que $\pi$ seja a distribuição de equilíbrio da cadeia.

Uma forma simples de fazermos isso é através de cadeias onde o núcleo $p$ satisfaça a condição de reversibilidade $\pi(\theta) p(\theta, \phi)=\pi(\phi) p(\phi, \theta), \quad \forall(\theta, \phi)$, sendo a condição suficiente para que $\pi$ seja a distribuição de equilíbrio da cadeia.

O núcleo $p(\theta, \phi)$ é constituído de dois elementos um núcleo de transiçāo $q(\theta, \phi)$ e uma probabilidade $\alpha(\theta, \phi)$ de forma que:

$$
p(\theta, \phi)=q(\theta, \phi) \alpha(\theta, \phi), \quad \text { se } \phi \neq \theta .
$$

Portanto, o núcleo de transição define uma densidade $p(\theta, \cdot)$ para todos os valores diferentes de $\theta$. Consequentemente, resta uma probabilidade positiva da cadeia ficar em $\theta$ dada por,

$$
p(\theta, \theta)=1-\int q(\theta, \phi) \alpha(\theta, \phi) \partial \phi
$$

Essas duas formas podem ser agrupadas na expressão geral:

$$
p(\theta, A)=\int_{A} q(\theta, \phi) \alpha(\theta, \phi) \partial \phi+I(\theta \in A)\left[1-\int q(\theta, \phi) \alpha(\theta, \phi) \partial \phi\right] .
$$


Logo, o núcleo de transição define uma distribuição mista para o novo estado $\phi$ da cadeia. Para $\phi \neq 0$, essa distribuição tem densidade e para $\phi=0$, essa distribuição atribui uma probabilidade positiva.

A expressão mais comumente citada para a probabilidade de aceitação é

$$
\alpha(\theta, \phi)=\min \left\{1, \frac{\pi(\phi) q(\phi, \theta)}{\pi(\theta) q(\theta, \phi)}\right\} .
$$

Assim, a simulação de uma amostra $\pi$ usando a cadeia de Markov definida pela transição (B.1) pode ser esquematizada da seguinte forma:

(i) inicializamos o contador de iterações da cadeia $j=1$ e arbitremos o valor inicial $\theta^{(0)}$

(ii) movemos a cadeia para um novo valor $\phi$ gerado da densidade $q\left(\theta^{(j-1)}, \cdot\right)$;

(iii) calculamos a probabilidade de aceitação do movimento $\alpha\left(\theta^{(j-1)}, \phi\right)$ dada por (B.2). Se o movimento for aceito $\theta^{(j)}=\phi$. Se não for aceito, $\theta^{(j)}=\theta^{(j-1)}$ e a cadeia não se move;

(iv) mudamos o contador de $j$ para $j+1$ e retornamos a (ii) até a convergência.

Realizamos a etapa (iii) após a geração de uma quantidade uniforme $u$ independentes de todas as outras variáveis. Se $u \leq \alpha$, o movimento é aceito e se $u>\alpha$ o movimento não é permitido. O núcleo de transição $q$ define uma proposta de movimento que pode ou não ser confirmado por $\alpha$. O sucesso do método depende de taxas de aceitação não muito baixas e de propostas fáceis de simular. O método substitui uma geração difícil de $\pi$ por várias geraçōes propostas de $(q)$. 


\section{Apêndice C}

\section{O CRITÉRIO DE}

\section{CONVERGÊNCIA DE GELMAN E}

\section{RUBIN (1992)}

Esta é uma forma simples de monitorar a convergência dos valores gerados para uma distribuição de equilíbrio.

O critério de convergência de Gelman e Rubin (1992) é baseado em técnicas de análise de variância. Para o algoritmo devernos considerar pelo menos duas cadeias paralelas com valores iniciais, diferentes, amostrados de uma distribuição bem comportada. Após as cadeias atingirem estacionariedade digamos na t-ésima iteração, consideramos as realizações $\left(\theta_{j}, \theta_{j+h}, \ldots, \theta_{j+n h}\right), \quad j \geq t$ como uma amostra aleatória da distribuição desejada. Devemos assumir $h$ razoavelmente grande de tal forma que 2 valores sucessivos de $\theta$ sejam independentes.

Se cada sequência tem tamanho $2 n$, descartamos as primeiras $n$ iterações e consideramos as $n$ restantes. Para cada parâmetro de interesse, calculamos:

- a variância entre as cadeias:

$$
E=\frac{n}{m-1} \sum_{i=1}^{m}\left(\bar{\theta}_{i}-\bar{\theta}\right)^{2}
$$

- a variância dentro das cadeias:

$$
D=\frac{1}{m(n-1)} \sum_{i=1}^{m} \sum_{j=1}^{n}\left(\theta_{i}^{(j)}-\bar{\theta}_{i}\right)^{2}
$$


onde: $\vec{\theta}_{i}$ é a média das observaçōes da $i$-ésima cadeia; e

$\bar{\theta}$ é a média dos $\bar{\theta}_{i} \operatorname{com} i=1,2, \ldots, m$.

Então, a variância da distribuição desejada pode ser estimada por $\widehat{\sigma}^{2}=\frac{n-1}{n} D+\frac{1}{n} E$ e a média por $\widehat{\mu}=\bar{\theta}$. Podemos melhorar na estimativa mais precisa da distribuição desejada, admitindo para a variabilidade amostral das estimativas, $\mu$ e $\widehat{\sigma}^{2}$. O resultado é uma distribuição $t$ de Student aproximada para $\theta$ com centro $\widehat{\mu}$, escala $\sqrt{\widehat{V}}=\sqrt{\widehat{\sigma}^{2}+E / m n} \mathrm{e}$ graus de liberdade $d f=2 \widehat{V}^{2} / \widehat{v a r}(\widehat{V})$, onde

$$
\begin{aligned}
\widehat{\operatorname{var}}(\widehat{V})= & \left(\frac{n-1}{n}\right)^{2} \frac{1}{m} \widehat{\operatorname{var}}\left(s_{i}^{2}+\left(\frac{m+1}{m n}\right)^{2} \frac{2}{m-1} E+\right. \\
& +2 \frac{(m+1)(n-1)}{m n^{2}} \frac{m}{n}\left[\widehat{\operatorname{cov}}\left(s_{i}^{2}, \bar{\theta}_{i}^{2}\right)-2 \bar{\theta} \widehat{\operatorname{cov}}\left(s_{i}^{2}, \bar{\theta}_{i}\right)\right],
\end{aligned}
$$

onde as variâncias e covariâncias estimadas são obtidas dos $m$ valores amostrais de $\bar{\theta}_{i}$ e $s_{i}^{2} ; d f \longrightarrow \infty$ quando $n \longrightarrow \infty$.

Monitoramos a convergência da simulação iterativa, calculando o fator de redução potencial de escala estimado $\sqrt{\widehat{R}}=\sqrt{(\widehat{V} / D) d f /(d f-2)}$. $\AA$ medida que $n$ cresce $(n \rightarrow$ $\infty), R$ converge para 1. $\widehat{R}$ é a razão do estimador da variância atual para a variância dentro da cadeia com um fator que avalia a variância adicional da distribuição 't de Student'.

Desde que $\widehat{R}$ esteja próximo de 1 , admitimos que a convergência ocorreu e as amostras das iterações selecionadas são independentes e identicamente distribuídas (ver, Gelman \& Rubin, 1992). 


\section{Apêndice D}

\section{PROGRAMA COMPUTACIONAL PARA O MODELO 3}

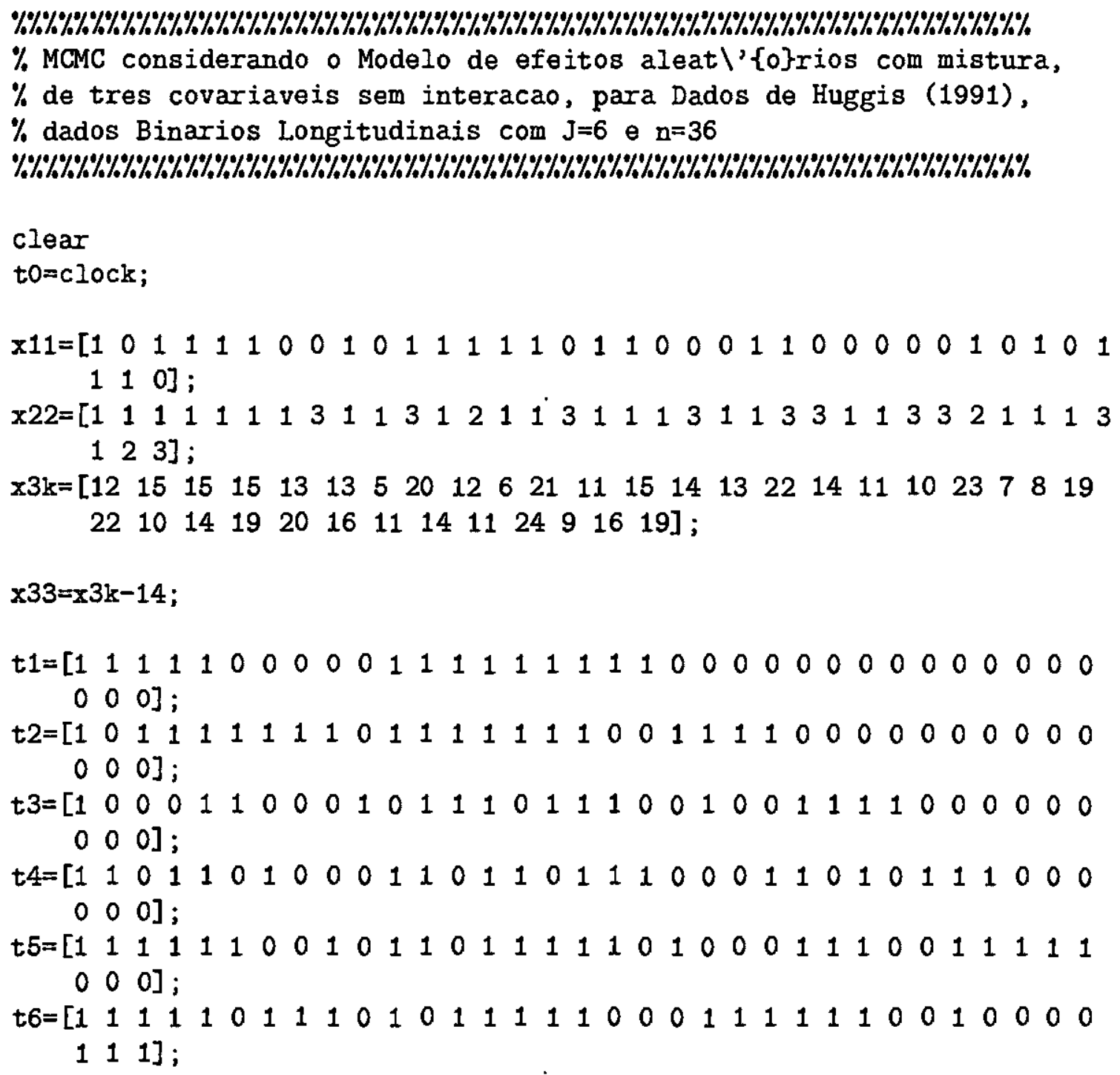




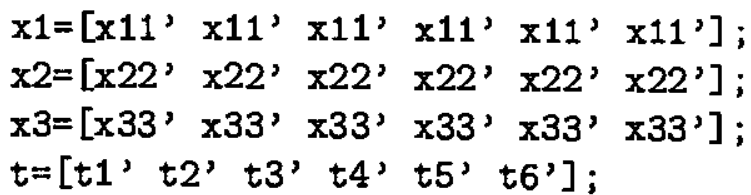

$[\operatorname{lin}, \operatorname{col}]=\operatorname{siz\theta }(t)$;

$\%$ Contadores

$c(1: 1$ in $)=0$. *ones $(1,1$ in $)$;

c1 $(1: \operatorname{col})=0 . *$ ones $(1$, col $)$;

$c 2(1: \operatorname{col})=0$.*ones $(1, \mathrm{col})$;

c3 $(1: \operatorname{col})=0$. *ones $(1, \operatorname{col})$;

$\mathrm{m}=5 \quad \%$ Numero de cadeias

int $=30001 \%$ Numero de iteracoes

\% condicoes iniciais

al_o=linspace $(-1,1, \mathrm{~m})$;

B1_o=linspace $(-5,4, \mathrm{~m})$;

p1_o=linspace $(.2, .8, \mathrm{~m})$;

mi1_o=linspace $(-.6,-.3, \mathrm{~m})$;

mi2_o=linspace $(.1, .8, \mathrm{~m})$;

sig1_0=linspace $(.02, .8, \mathrm{~m})$;

sig2_o=sig1_o;

for $i=2$ :int $\quad \%$ LOOP para iteracoes

$f=1 ; g=1 ; \quad \% \in$ para $p 1 ; g$ para $p 1$

$\mathrm{a} 1=4 ; \mathrm{b} 1=3 ; \quad \%$ priori p/ sig1

$\mathrm{a} 2=\mathrm{a} 1 ; \mathrm{b} 2=\mathrm{b} 1 ; \quad \%$ priori $\mathrm{p} / \mathrm{sig2}$

$\mathrm{d} 1=-0.4 ; \quad \theta 1=0.2 ; \%$ priori p/mi1.

$\mathrm{d} 2=0.4 ;$ e2=0.2; \% priori $\mathrm{p} / \mathrm{mi2}$

if $\operatorname{rem}(i-2,6000)==0$

alf $(i-1,1: \operatorname{lin})=a I_{-} 0(1+(i-2) / 6000)$. *ones $(1,1$ in $)$;

$B 1(i-1,1: \mathrm{col})=B 1+0(1+(i-2) / 6000)$. *ones $(1, \mathrm{col})$;

$B 2(i-1,1: c 01)=B 1 \_0(1+(i-2) / 6000)$. *ones $(1$, col $)$;

$B 3(i-1,1: c 01)=B 1 \_o(1+(i-2) / 6000)$. *ones $(1, c 01)$;

$\mathrm{p} 1(i-1)=\mathrm{p} 1 \_0(1+(i-2) / 6000)$;

$\operatorname{mi1}(i-1)=\operatorname{mi1} \_o(1+(i-2) / 6000)$;

$\operatorname{mi2}(i-1)=\operatorname{mi2} \_0(1+(i-2) / 6000)$;

$\operatorname{sig} 1(i-1)=\operatorname{sig} 1 \_o(1+(i-2) / 6000)$;

end

$\operatorname{sig} 2(i-1)=\operatorname{sig} 2 \_0(1+(i-2) / 6000)$; 


\section{$\% * * * * * * *$ CALCULOS BASICOS E GERACAO DA z1 ********}

for is $=1: \operatorname{lin}$

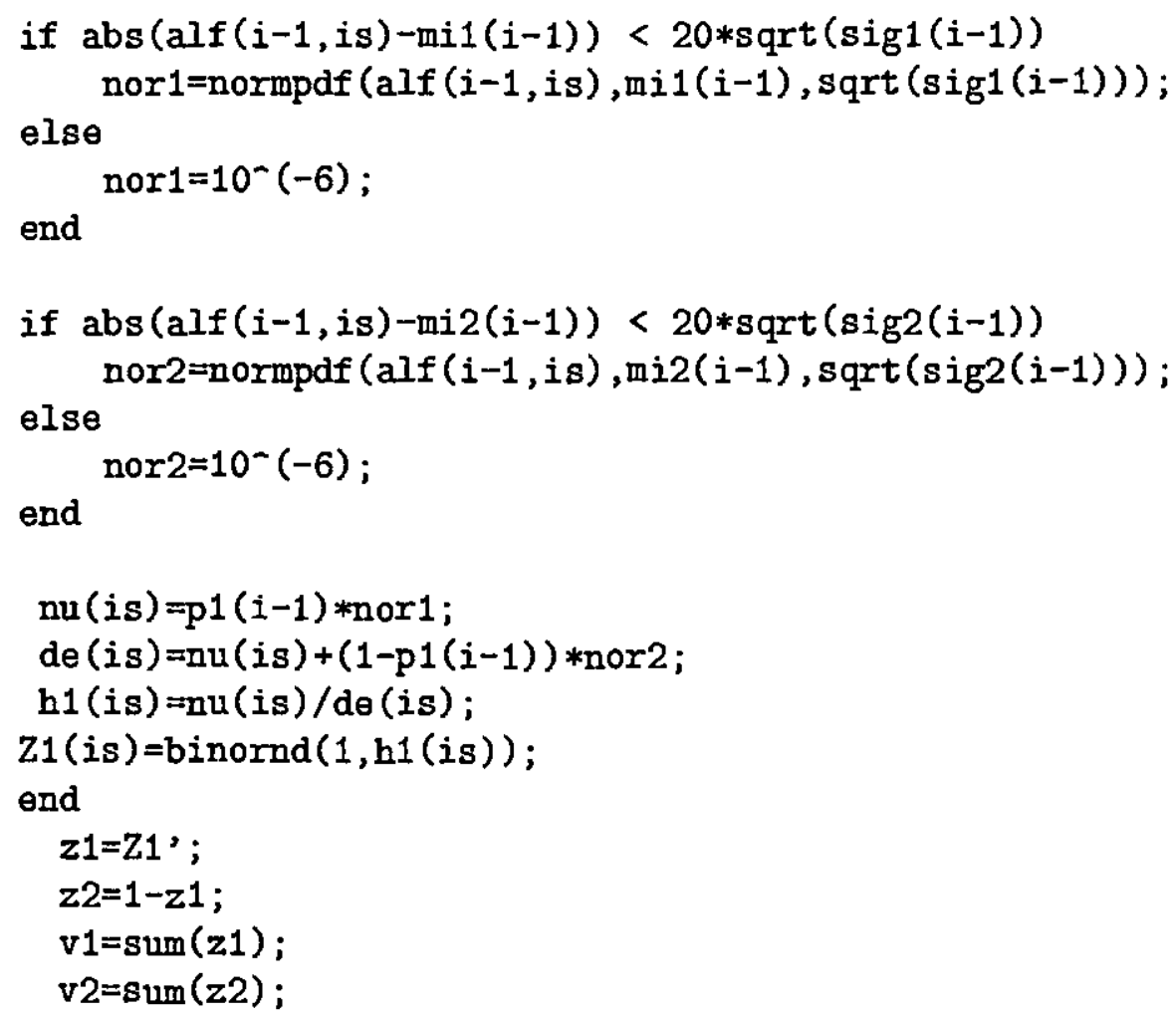

end

\%********** Metropolis para alpha ***************

for $s=1: 1$ in

$\mathrm{MU}(\mathrm{s})=(z 1(\mathrm{~s}) * \operatorname{mi1}(i-1) * \operatorname{sig} 2(i-1)+z 2(s) * \operatorname{mi} 2(i-1) * \operatorname{sig} 1(i-1)) / \ldots$ $(z 1(s) * \operatorname{sig} 2(i-1)+z 2(s) * \operatorname{sig} 1(i-1))$;

$\operatorname{sIG}(s)=(\operatorname{sig} 1(i-1) * \operatorname{sig} 2(i-1)) /(z 1(s) * \operatorname{sig} 2(i-1)+z 2(s) * \operatorname{sig} 1(i-1))$; 
$\operatorname{all}=\operatorname{normrnd}(\operatorname{MU}(s), \operatorname{sqrt}(\operatorname{SIG}(s)))$;

psio_al=exp $(a l f(i-1, s) * \operatorname{sum}(t(s,:))-\operatorname{sum}(\log (1+\exp (a l f(i-1, s)+m 1(s,:) \ldots$

$+\mathrm{m} 2(\mathrm{~s},:)+\mathrm{m} 3(\mathrm{~s},:)))))$;

psin_al $=\exp (a l l * \operatorname{sum}(t(s,:))-\operatorname{sum}(\log (1+\exp (\operatorname{all}+m 1(s,:)+m 2(s,:)+m 3(s,:)))))$;

p_all=min (psin_al/psio_al, 1);

\% Crit \'\{e\}rio de aceitacao

$\mathrm{u}=\mathrm{rand}$;

if $\mathrm{u}<\mathrm{p}_{-} \mathrm{all}$

alf $(i, s)=a 11$;

$c(s)=c(s)+1$;

else

$\operatorname{alf}(i, s)=\operatorname{alf}(i-1, s)$;

end

end

$\left.a]=[a] f(i,:)^{\prime}\right]$;

\%************* Gibbs para mil *********

MI1 $=(d 1 * \operatorname{sig} 1(i-1)+e 1 * \operatorname{sum}(\operatorname{al} . * z 1)) / \ldots$

( $\operatorname{sig} 1(i-1)+e 1 * v 1)$;

$\mathrm{SG} 1=(e 1 * \operatorname{sig} 1(i-1)) /(\operatorname{sig} 1(i-1)+\theta 1 * v 1)$;

$\operatorname{mi1}(i)=$ normrnd $(M I 1, \operatorname{sqxt}($ SG1) );

\%************* Gibbs para mi2 *********

MI2 $=(d 2 * \operatorname{sig} 2(i-1)+e 2 * \operatorname{sum}(a l . * z 2)) / \ldots$

( $\operatorname{sig} 2(i-1)+e 2 * v 2)$;

$S G 2=(e 2 * \operatorname{sig} 2(i-1)) /(\operatorname{sig} 2(i-1)+e 2 * v 2)$;

$\operatorname{mi2}(i)=\operatorname{normmnd}(M I 2$, sqxt $(S G 2))$;

\%************* Gibbs para sigma2_1 *********

$\mathrm{Aa1}=\mathrm{a} 1+\mathrm{v} 1 / 2$;

$\mathrm{Bb} 1=\left(1 / \mathrm{b} 1+\operatorname{sum}\left(z 1 . *(a I-\operatorname{mi1}(i)) \cdot{ }^{\sim} 2\right) / 2\right)^{\sim}(-1)$;

$\operatorname{sig} 1(i)=1 /(\operatorname{gamrnd}(\mathrm{Aa1}, \mathrm{Bb} 1))$;

\%************* Gibbs para sigma2_2 *********

Aa2 $=a 2+v 2 / 2$; 


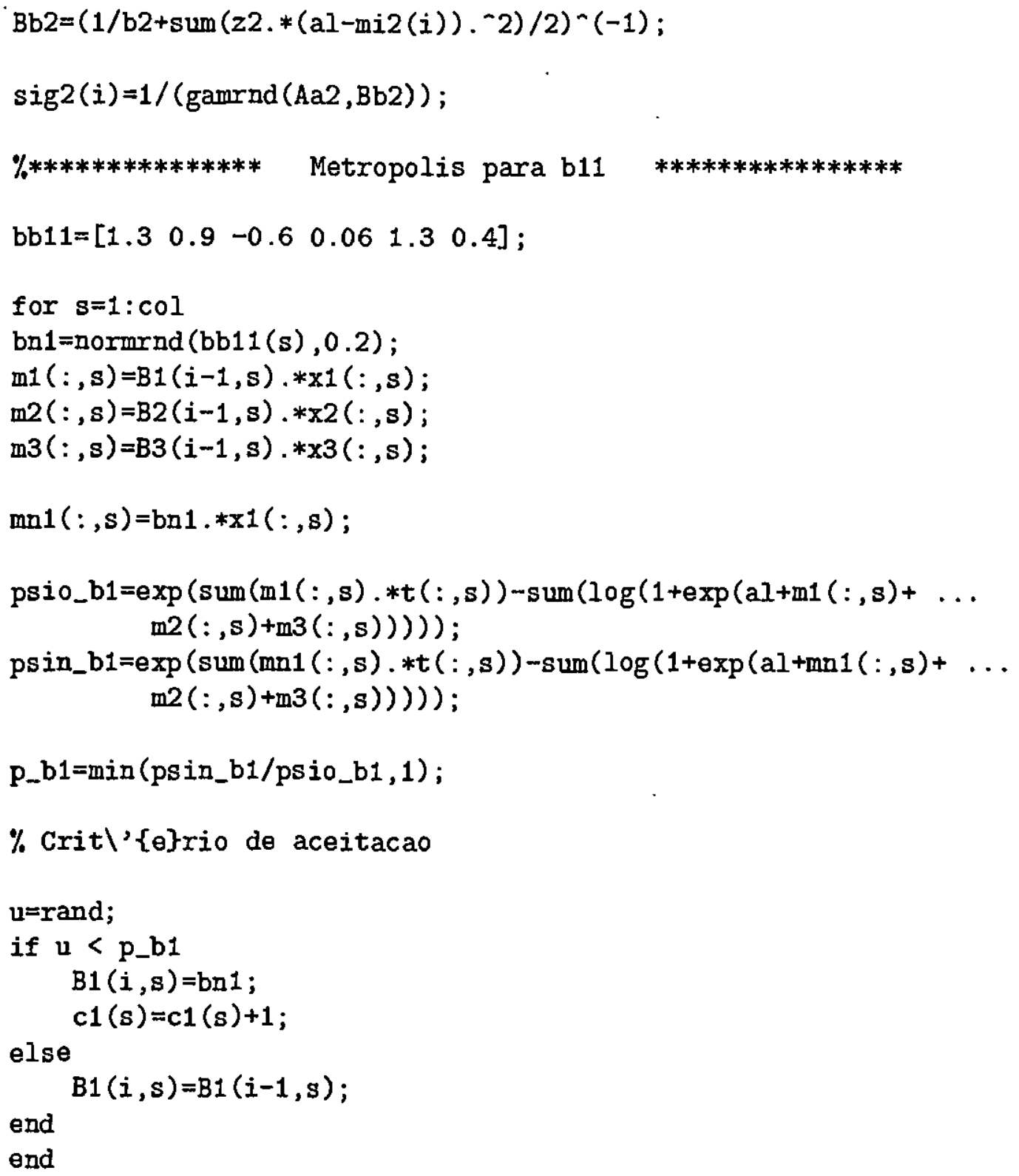


$\mathrm{m} 2(:, \mathrm{s})+\mathrm{m} 3(:, \mathrm{s})))))$;

psin_b2 $=\exp (\operatorname{sum}(\operatorname{mn} 2(:, s) . * t(:, s))-\operatorname{sum}(\log (1+\exp (a)+m 1(:, s)+\ldots$ $m n 2(:, s)+m 3(:, s))))$ );

p_b2=min (psin_b2/psio_b2,1);

\% Crit\'\{e\}rio de aceitacao

$\mathrm{u}=\mathrm{rand}$;

if $\mathrm{u}<\mathrm{p}$-b2

$\mathrm{B} 2(i, \mathrm{~s})=\mathrm{bn} 2$;

$\mathrm{c} 2(\mathrm{~s})=\mathrm{c} 2(\mathrm{~s})+1$;

else

$\mathrm{B} 2(i, s)=\mathrm{B} 2(i-1, s)$;

end

end

\%*************** Metropolis para b3

$b b 33=\left[\begin{array}{llllll}0.3 & 0.1 & 0.08 & 0.1 & 0.3 & 0.1\end{array}\right] ;$

for $s=1: \operatorname{col}$

bn3=normrnd $(b b 33(s), 0.2)$;

$\mathrm{m} 1(:, \mathrm{s})=\mathrm{B} 1(\mathrm{i}, \mathrm{s}) . * \mathrm{x} 1(:, \mathrm{s})$;

$\mathrm{m} 2(:, s)=\mathrm{B} 2(i, s) . * \times 2(:, s)$;

$\mathrm{m} 3(:, \mathrm{s})=\mathrm{B} 3(i-1, \mathrm{~s}) . * \mathrm{x} 3(:, \mathrm{s})$;

$m n 3(:, s)=b n 3 . * x 3(:, s)$;

psio_b3 $=\exp (\operatorname{sum}(m 3(:, s) . * t(:, s))-\operatorname{sum}(\log (1+\exp (a l+m 1(:, s)+\ldots$ $\mathrm{m} 2(:, s)+m 3(:, s)))))$;

psin_b3 $=\exp (\operatorname{sum}(\operatorname{mn} 3(:, s) . * t(:, s))-\operatorname{sum}(\log (1+\exp (a l+m 1(:, s)+\ldots$ $\mathrm{m} 2(:, \mathrm{s})+\mathrm{mn} 3(:, \mathrm{s})))))$;

p_b3=min(psin_b3/psio_b3,1);

\% Critl'\{e\}rio de aceitacao

$\mathrm{u}=\mathrm{rand}$;

if $u<$ p_b3

$\mathrm{B} 3(i, s)=b n 3$;

$c 3(s)=c 3(s)+1$

else

end

$\mathrm{B} 3(i, s)=\mathrm{B} 3(i-1, s)$;

end 
end $\% \mathrm{p} /$ iteracoes

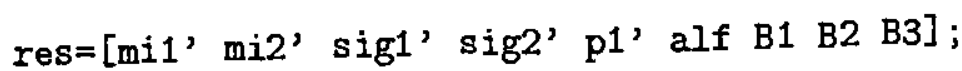

\% Selecao das Amostras

$g=1 ;$

for $j=3001: 6000: 30001$

for $k=j: j+2999$

if $\operatorname{rem}(k-j+1,10)==0$

res_.s $(g,:)=\operatorname{res}(k,:)$;

end

$\mathrm{g}=\mathrm{g}+1$;

end

end

amostra=length (res_s $(:, 1))$

$\% \% \% \% \% \% \% \% \% \% \% \% \% \% \% \% \% \% \% \% \% \% \% \% \% \% \% \% \% \% \% \% \% \% \% \% \% \%$

save $\mathrm{m} 3 \mathrm{n} 36$ res

save m3n36_s res_s

$\% \% \% \% \% \% \% \% \% \% \% \% \% \% \% \% \% \% \% \% \% \% \% \% \% \% \% \% \% \% \% \% \% \% \% \% \% \% \% \%$

$\%$ Inferencias

M_res-mean (res_s);

S_res =std (res_s);

Me_res=median (res_s);

IC95_resin=prctile (res_s, 2.5);

IC95_ressu=prctile (res_s, 97.5);

\%****** Analise de Convergencia Gelman \& Rubin

$[L, C]=s i z e\left(r e s_{-} s\right)$;

for $j=1: C$

for $s=1: m$

$M(:, s)=r e s \_s(1+((s-1) *(L)) / m:(s *(L)) / m, j)$;

end

$G R(j)=c c-g r(M)$;

end

Tempo $=e t i m e(c l o c k, t 0)$

Tempo

$\mathrm{ta}=[3000030000300003000030000 \mathrm{c}$ c1 c2 c3]./int;

ind $=[[1: 5][1: 36][1: 6][1: 6][1: 6]]$;

sol $=[$ ind' M_res' S_res' IC95_resin' IC95_ressu' $G R$ ' ta'] 


\section{Referências Bibliográficas}

[1] AMEMTYA, T. Advance Econometrics. Harvard University Press. Boston, 1985. p.535-546, 1970. Longitudinal Binary Data Gibbs Sampler For The Logistic Mddel

[4] BICKEL, P. Dtatistics in Medicine? 0.17 , p.2905 in the Analysis of $1977 . \quad$ J.j DOKSUM, K.A. Mathematicals Metropolis Samplers. The Journal of Computationatond Gibbs, Hit-and-Run, and
p.251-272, 1993 .
COX, D.R. The Analysical Statistici,

[7] CHIB, S.; GREENBER of Binary Data. London, Chapan and Hall, 1970.

[8] CHIB, S. Olin School of Business, Washington Uninersiate Probit Models. Tech 347-361, 1998.

[11] Ger Report, Dep. of Statistician Analysis of Cot Marginal Den.E.; SMITH, A

Journal of the Sampling-Based, Appro Statistical Association, 0.85 , pulating

[13] GELMAN, A.147-167, 1992.
1ntional Meeting on Bay-Based Methods. Using Predictive

Sequences. A.; RUBIN, B.D. Inference fromesian Statistics, Oxocedings of the
Statistical Scienci University 
[14] GEMAN, S.; GEMAN,
Bayesian Restoration

Bayesian Restoration of D. Stochastic Relaxation, Gibbs Distributions and the
chine Intelligence, n.6, p.721-741, IEEE Transactions on Pattern Analysis and.MaCambridge University Press, 1995.

[16] HASEMAN, J.K.; KUPPER, L.J. Analysis of Dichotomus Response Data from
Certain Toxicological Experimentes. Biometrics, n.35, p.281-293, 1979.
17] HOSMER, D.W.; LEMESHOW, S. Applied Logistic Regression. New York, John
Wiley and Sons, 1989.

[19] KASS, R.E. e RAFTERY, A.E. Payes Factors. Journal of the American Statistical
Association, n.90, p.773-795, 1945, Association, n.90, p.T73-795, 1945, Models. Biometrika, n.73, p.13-22, 1986. ed Likelihood Bootstrip. Joural of the Royal Statistical Society, series B, n.56, p.3[22] OCHI, Y. e PRENTICE, R.IL Likelihood Inference in a Correlated Probit Regression
Model.Biometrics, n.71, p.5pt-543, 1984 .

[23] PRENTICE, R.L. Correlaad Binary Regression with Covariate Specific to Each Binary Observation.Biome ics, n.44, p.1033-1048, 1988.

[24] RAFTERY, A.E. Hypothedis Testing and Model Selection. In Markov Chain Monte Carlo in practice, W. Gilts, S. Richardson and D.J. Spiegelhalter, eds., London, Chapman and Hall, p.163-187, 1996.

[25] RAO, C.R. Linear Statisfical Inference and its Applications. 2.ed. New York, Wiley, 1973.

[26] SOARES, J.F.; COLOSIMO, E.A. Métodos Estatísticos na Pesquisa Clínica. Ribeirão Preto, SP, USP, 1995.

[27] TANNER, M.; WONG, W. The Calculation of Posterior Distributions by Data Augmentation. Journal of the American Statistical Association, n.82, p.528-550, 1987.

[28] ZEGER, S.L.; LIANG, K.Y. Longitudinal Data Analysis for Discrete and Continuous Outcomes. Biometrics, n.42, p.121-130, 1986. 